\title{
REPRESENTATIONS OF COMPACT GROUPS ON BANACH ALGEBRAS
}

$\mathrm{BY}$

\author{
DAVID GURARIE
}

\begin{abstract}
Let a compact group $U$ act by automorphisms of a commutative regular and Wiener Banach algebra $A$. We study representations $R^{\omega}$ of $U$ on quotient spaces $A / I(\omega)$, where $\omega$ is an orbit of $U$ in the Gelfand space $X$ of $A$ and $I(\omega)$ is the minimal closed ideal with hull $\omega \subset X$. The main result of the paper is: if $A=A_{\rho}(X)$ is a weighted Fourier algebra on a LCA group $X=\hat{A}$ with a subpolynomial weight $\rho$ on $A$, and $U$ acts by affine transformations on $X$, then for any orbit $\omega \subset X$ the representation $R^{\omega}$ has finite multiplicity. Precisely, the multiplicity of $\pi \in \hat{U}$ in $R^{\omega}$ is estimated as $k\left(\pi ; R^{\omega}\right) \leq c \cdot \operatorname{deg}(\pi) \forall \pi \in \hat{U}$ with a constant $c$ depending on $A$ and $\rho$.

Applications of this result are given to topologically irreducible representations of motion groups and primary ideals of invariant subalgebras.
\end{abstract}

Introduction. Let $(A, U)$ be a pair of commutative Banach algebra $A$ and a compact group $U$ acting by automorphisms on $A$. We shall always assume that $A$ is regular and Wiener (Tauberian) on its Gelfand space $X=\hat{A}$ (see $[\mathbf{1 1}$, Chapter 10]), and the representation $a \rightarrow a^{u}(a \in \mathcal{A})$ of $U$ on $\mathcal{A}$ is strongly continuous.

There are two other algebras associated with any such pair: the crossed product, or generalized (twisted) $L^{1}$-algebra $\mathcal{L}=\mathcal{L}(U ; \mathcal{A})$ and the subalgebra of $U$-invariants, $Z=A^{U}$.

Algebras $\mathcal{L}=\mathcal{L}(U ; \mathcal{A})$ naturally appear in the study of group extensions $[\mathbf{1}$, 13]. Namely, if a locally compact group $G=A \rtimes U$ is a semidirect product of a normal subgroup $A$ and a subgroup $U$ acting on $A$ by automorphisms, then the group algebra $L^{1}(G)$ is isomorphic to the crossed product $\mathcal{L}\left(U ; L^{1}(A)\right)$. In some cases (for instance, if $U$ is compact) more general weighted group algebras $L_{\rho}^{1}(G)$ can also be realized as crossed products, $L_{\rho}^{1}(G) \simeq \mathcal{L}\left(U ; L_{\rho \prime}^{1}(A)\right)$, where $\rho^{\prime}=\rho \mid A$ (see $[\mathbf{1 0}]$ ).

For abelian groups $A$ and compact groups $U$ semidirect products $G=A \rtimes$ $U$ are called motion groups. Correspondingly, crossed products $\mathcal{L}=\mathcal{L}(U, \mathcal{A})$ of commutative Banach algebras $\mathcal{A}$ by compact groups $U$ were called motion algebras in $[\mathbf{1 4}]$.

In harmonic analysis of motion algebras $\mathcal{L}$ and subalgebras $Z=A^{U}$ an important role is played by $U$-invariant ideals of $\mathcal{A}$ (i.e., $I^{u}=I, \forall u \in U$ ). Thus in many cases

Received by the editors March 11, 1982 and, in revised form, January 17, 1983 and August 23, 1983.

1980 Mathematics Subject Classification. Primary 43A20, 43A65, 22D12; Secondary 22C05, $22 B 10$.

Key words and phrases. Compact, locally compact abelian, motion groups; Banach, regular, Wiener, Beurling (weighted), Fourier, Lie algebras; Banach, induced, finitely multiplied, *-, topologically irreducible representations; Banach bundles, tangent and normal bundles; jets; Whitney functions; $m$-smooth vectors; affine transformations; minimal and primary ideals; module; Whitney's Theorem; Frobenius reciprocity theorem. 
$U$-invariant ideals of $A$ are in 1-1 correspondence with ideals of $Z$. On the other hand, each invariant ideal $I$ gives rise to the ideal $\mathcal{N}=\mathcal{L}(U ; I)$ of the crossed product $\mathcal{L}$.

Obviously, the hull (or spectrum) $\sigma(I)$ of a $U$-invariant ideal $I \subset A$ is a closed subset invariant under the dual action of $U$ on $X$. Conversely, to any $U$-invariant closed subset $\Delta \subset X$ there correspond two $U$-invariant ideals of $A, I(\Delta)$ the minimal ideal with the hull $\Delta$ and $J(\Delta)$ the maximal.

We shall be mainly concerned with ideals $J(\omega)$ and $I(\omega)$ which correspond to smallest possible hulls $\omega \subset X$, i.e., orbits of the group $U$ in $X$. To any such $\omega$ we attach a representation $R^{\omega}$ of the group $U$ on the quotient space $A / I(\omega)$.

Following $[\mathbf{1 0}]$ we consider the condition that $R^{\omega}$ is a representation of finite multiplicity (FM), which means that each irreducible $\pi \in \hat{U}$ occurs in $R^{\omega}$ with a finite multiplicity

$$
k\left(\pi ; R^{\omega}\right)<\infty \quad \forall \pi \in \hat{U} .
$$

We also introduce a stronger condition

$$
k\left(\pi ; R^{\omega}\right) \leq c d(\pi) \quad \forall \pi \in \hat{U}
$$

with some constant $c>0$. Here $d(\pi)$ denotes the degree of $\pi$. In other words the representation $R^{\omega}$ is "bounded" by a finite multiple of the regular representation.

The representation $R^{\omega}$ (precisely, the constant $c=c(\omega)$ ) can serve as the measure of the defect of spectral synthesis for $I(\omega)$.

Another measure of the defect of spectral synthesis is given by the so-called m-synthesizibility condition

$$
J(\omega)^{m} \subseteq I(\omega) \text { for some } m=m(\omega)<\infty .
$$

Notice that in the case of the trivial action of $U$ on $X$, i.e., one-point orbits $\omega \subset X$, both conditions reduce to a generalized form of Ditkin's property,

$$
\operatorname{dim} A / I(x)<\infty \text { for any minimal primary ideal } I(x) \text {. }
$$

Conditions (S) and (R) were used in $[\mathbf{1 4}$ and 10] in the study of ideals and representations of motion algebras. In particular one of the results [14] states: if $A$ is a ${ }^{*}$-algebra and a pair $(A, U)$ satisfies (S), then the only prime ideals of the motion algebra $\mathcal{L}=\mathcal{L}(U ; A)$ are ${ }^{*}$-primitive ideals (i.e., kernels of irreducible *-representations of $\mathcal{L}$ ).

An interesting class of prime ideals are kernels of topologically irreducible Banach representations of $\mathcal{L}$. The results of $[\mathbf{1 4}]$ imply in particular that any topologically irreducible representation $T$ of a motion algebra $\mathcal{L}$ satisfying property $(\mathrm{S}) \backslash$ factors through a representation of the quotient algebra $\mathcal{L} / \mathcal{M}$, where $\mathcal{M}$ is a ${ }^{*}$-primitive ideal. Since quotients $\mathcal{L} / \mathcal{M}$ of motion algebras $\mathcal{L}$ have sufficiently "nice" structure e.g., $\mathcal{L} / \mathcal{M}$ possesses "enough small idempotents" (cf. $[\mathbf{4}, \mathbf{7}])$ one can derive many nice properties of $T$. Namely $[\mathbf{1 0}]$, any topologically irreducible representation $T$ of $\mathcal{L}$ is completely irreducible, $T$ has an algebraically irreducible dense restriction, $T$ is Naimark-equivalent to an irreducible *-representation, etc.

In $[\mathbf{1 0}]$ we proved the last three statements also for motion algebras $\mathcal{L}=\mathcal{L}(U ; \mathcal{A})$ with pairs $(A, U)$ having finitely multiplied representations $R^{\omega}$.

The following examples illustrate the nature of representations $R^{\omega}$ and properties (R) and (S). 
EXAMPLE 1. Let $I(\omega)=J(\omega)$. In terms of harmonic analysis this means that $\omega$ is a set of spectral synthesis.

Obviously, property (S) holds with $m=1$.

The Gelfand homomorphism identifies $A / I(\omega)$ with a subspace of the space of continuous function $C(\omega)$. So $R^{\omega}$ turns out to be a subrepresentation of the regular representation $R$ of $U$ on the homogeneous space $\omega \simeq U_{x} \backslash U$ (U modulo the stabilizer of a point $x \in \omega$ ). The representation $R$ is induced by the trivial representation $\mathbf{1}$ of $U_{x}, R=\operatorname{Ind}_{U_{x}}^{U}(\mathbf{1})$. By the well-known Frobenius reciprocity theorem, for any $\hat{\pi} \in U$ the multiplicity of $\pi$ in $R$,

$$
k(\pi ; R)=k\left(\pi \mid U_{x} ; \mathbf{1}\right) \leq d(\pi)
$$

Then of course,

$$
k\left(\pi ; R^{\omega^{\prime}}\right) \leq k(\pi ; R) \leq d(\pi) \quad \forall \pi \in \hat{U},
$$

i.e., $R^{\omega}$ is finitely multiplied.

ExAmple 2 (N. VAROpoulos $[\mathbf{2 4}])$. Let $A=L^{1}\left(\mathbf{R}^{n}\right)$, and let $U=S O(n)$ act on $A$ by the change of variable

$$
u: f(x) \rightarrow f\left(x^{u}\right) \quad\left(x \in \mathbf{R}^{n} ; u \in U\right) .
$$

Here orbits $\omega \subset \mathbf{R}^{n}$ are spheres of radius $r \geq 0$. For any nonzero $\omega$ the minimal ideal $I(\omega) \neq J(\omega)$ unless $n=2$.

According to [24], there exists a decreasing chain of $U$-invariant ideals,

$$
J(\omega)=J_{1}(\omega) \supset J_{2}(\omega) \supset \cdots \supset J_{m}(\omega)=I(\omega) \quad(m=[(n+1) / 2]),
$$

s.t. $J_{k}(\omega)=J(\omega)^{k}$.

Thus property (S) holds with $m=[(n+1) / 2]$. Moreover, it can be derived from [24] that the representation $R^{\omega}$ is equivalent to a multiple of the regular representation $R$ of $U$ on $\omega$, namely,

$$
R^{\omega} \sim R \otimes[(n+1) / 2] .
$$

Since in this case the multiplicities of $R$ are well known $(k(\pi ; R)=1$ or 0$)$ we get

$$
k\left(\pi ; R^{\omega}\right) \leq m=[(n+1) / 2] \quad \forall \pi \in \hat{U}
$$

This implies property $(\mathrm{R})$ for all pairs $\left(L^{1}\left(\mathbf{R}^{n}\right), S O(n)\right)$.

EXAMPLE 3. Let $A=C^{m}\left(\mathbf{R}^{n}\right)$ be the algebra of $m$-smooth functions on $\mathbf{R}^{n}$ (or more general on a smooth manifold $X$ ) with the usual sequence of $m$-seminorms

$$
\|f\|_{m, Q}=\sum_{|\nu| \leq m}\left\|\partial^{\nu} f\right\|_{L^{\infty}(Q)} .
$$

Here $\nu=\left(\nu_{1}, \ldots, \nu_{n}\right)$ is a multi-index, $|\nu|=\nu_{1}+\cdots+\nu_{n}, \partial^{\nu}$ the corresponding partial derivative and $Q$ runs over all compact subsets of $\mathbf{R}^{n}$. We assume that a compact group $U$ acts smoothly on $\mathbf{R}^{n}$ and consider an orbit $\omega \subset \mathbf{R}^{n}$. The minimal ideal $I(\omega)$ of $C^{m}\left(\mathbf{R}^{n}\right)$ is characterized by the well-known Whitney's Theorem [26]

$$
I(\omega)=\left\{f \in C^{m}\left(\mathbf{R}^{n}\right)\left|\left(\partial^{\nu} f\right)(x)=0 \forall x \in \omega,\right| \nu \mid \leq m\right\} .
$$

Equality (3) holds for any closed subset $\omega \subset \mathbf{R}^{n}$ and in terms of harmonic analysis it means that the minimal ideal $I(\omega)$ coincides with the intersection of 
all primary ideals which contain it, i.e., $I(\omega)$ is synthesizible. Actually, Whitney's theorem established synthesizibility in the above sense of all closed ideals $I \subset$ $C^{m}\left(\mathbf{R}^{n}\right)$. An immediate consequence of (3) is property (S) for minimal ideals $I(\omega) \subset C^{m}\left(\mathbf{R}^{n}\right)$ with $m(\omega)=m+1$ (the degree of smoothness +1$)$.

Let us study the representation $R^{\omega}$. By (3) one can attach to each element $\tilde{f} \in$ $A / I(\omega)\left(f \in \mathcal{A}=C^{m}\left(\mathbf{R}^{n}\right)\right.$ a tuple of partial derivatives $J^{m}(f, x)=\left\{\partial^{\nu} f(x)\right\}_{|\nu| \leq m}$, known as the $m$-jet of $f$, restricted on $\omega$,

$$
W: \tilde{f} \rightarrow J^{m}(f, x) \quad(x \in \omega) .
$$

The operator $W$ embeds $A / I(\omega)$ into the space of all continuous (vector-valued) jet functions on $\omega$. One can easily verify (see $[\mathbf{1 0}]$ ) that $W$ intertwines the representation $R^{\omega}$ and the induced representation $R=\operatorname{Ind}_{U_{x}}^{U}\left(\tau_{m}\right)$, where $U_{x}$ is a stability subgroup of a point $x \in \omega$. The inducing representation $\tau_{m}$ is described as follows. The differential $d_{x} u$ of a smooth map $u: x \rightarrow x^{u}\left(x \in \mathbf{R}^{n}, u \in U\right)$ is a linear operator on tangent spaces

$$
d_{x} u: T_{x} \simeq \mathbf{R}^{n} \rightarrow T_{x^{u}} \simeq \mathbf{R}^{n},
$$

and the family of differentials $\{d u\}_{u \in U}$ obey the "chain rule".

In particular, for elements $u$ of a stabilizer $U_{x}$ we get a representation $\tau$ of $U_{x}$ on the tangent space $T_{x} \simeq \mathbf{R}^{n}$. The representation $\tau$ extends to the space of all symmetric $k$-tensors over $\mathbf{R}^{n}, S_{k}\left(\mathbf{R}^{n}\right)(k=0,1, \ldots)$, and thus gives rise to a representation $\tau_{m}$ of $U$ on the space of $m$-jets, $J^{m}\left(\mathbf{R}^{n}\right)=\bigoplus_{k=0}^{m} S_{k}\left(\mathbf{R}^{n}\right)$.

By the Frobenius reciprocity theorem the induced representation $R=\operatorname{Ind}_{U_{x}}^{U}\left(\tau_{m}\right)$ and therefore a subrepresentation $R^{\omega} \subset R$, is FM, and the multiplicities

$$
k\left(\pi ; R^{\omega}\right) \leq k(\pi ; R)=k\left(\pi \mid U_{x}, \tau_{m}\right) \leq d\left(\tau_{m}\right) \cdot d(\pi) .
$$

Formula (4) establishes the equivalence of $R^{\omega}$ with a subrepresentation of an induced representation. This result can be sharpened and $R^{\omega}$, as in the second example, is actually equivalent to an induced representation, $R^{\omega} \sim \operatorname{Ind}_{U_{x}}^{U}\left(\tau_{m}^{\prime}\right)$. This follows from the results of $\S 5$ of our paper (Theorem 3 and Lemma 6 ). The inducing representation $\tau_{m}^{\prime}$ acts on a subspace of $J^{m}\left(\mathbf{R}^{n}\right)$, which consists of all "transversal (normal) derivatives" at $x \in \omega$ (of course this space is smaller than $J^{m}\left(\mathbf{R}^{n}\right)$ ). To describe $\tau_{m}^{\prime}$ explicitly we notice that the representation $\tau(u): \mathbf{R}^{n} \rightarrow \mathbf{R}^{n}\left(u \in U_{x}\right)$ leaves invariant the tangent space of $\omega$ at $x, T_{x}(\omega)$, considered as a subspace of $\mathbf{R}^{n}$. By Weyl's principle of complete reducibility $T_{x}(\omega)$ possesses a $\tau$-invariant complement $T_{x}^{\prime}$ in $\mathbf{R}^{n}$ : We denote by $\tau^{\prime}$ the restriction $\tau \mid T_{x}^{\prime}$ and by $\tau_{m}^{\prime}$ the extension of $\tau^{\prime}$ to the space of all "transversal $m$-jets", $J^{m}\left(T_{x}^{\prime}\right)=\bigoplus_{k=0}^{m} S_{k}\left(T_{x}^{\prime}\right)$. Here, as above, $S_{k}\left(T_{x}\right)$ denotes the space of symmetric $k$-tensors over $T_{x}^{\prime}$. Then

$$
R^{\omega} \sim \operatorname{Ind}_{U_{x}}^{U}\left(\tau_{m}^{\prime}\right)
$$

In particular, the multiplicities

$$
k\left(\pi ; R^{\omega}\right)=k\left(\pi \mid U_{x} ; \tau_{m}^{\prime}\right) \leq d\left(\tau_{m}^{\prime}\right) d(\pi),
$$

which gives better estimates than (5). Indeed, the degree of $\tau_{m}, d\left(\tau_{m}\right)=\left(\begin{array}{c}n+m \\ m\end{array}\right)$, depends on $n=\operatorname{dim} \mathbf{R}^{n}$, while $d\left(\tau_{m}^{\prime}\right)=\left(\begin{array}{c}k+m \\ m\end{array}\right)$ depends only on $k=n-\operatorname{dim} \omega$.

EXAMPLE 4. Let $A=A(X)$ be the Fourier algebra on a compact abelian group $X$ and let a closed subgroup $Y \subset X$ act by translations on $X$. The orbits $\omega$ are 
cosets in $X$. As is well known [20] any closed coset of a LCA group $X$ is a set of spectral synthesis for $A(X)$. Hence both property (S) with $m=1$ and $(\mathrm{R})$ with $c(\omega)=1$ hold. Moreover, $R^{\omega}$ is equivalent to the regular representation of $Y$ on $A(Y) \simeq A(X) / I(Y)$.

So in all the above examples we get both property $(S)$ and $(R)$. Moreover, the representation $R^{\omega}$ is induced by a finite dimensional representation and the multiplicities $k\left(\pi ; R^{\omega}\right)$ are estimated as follows:

$$
k\left(\pi ; R^{\omega}\right) \leq c \cdot d(\pi) \quad \forall \pi \in \hat{U}
$$

with some constant $c$.

Thus we are led to the following conjectures.

Let $(A, U)$ be a pair of a commutative regular and Wiener Banach algebra $A$ and a compact group $U$ acting on $A$. We assume that $A$ satisfies property $(\mathrm{F})$, $\operatorname{codim} I(x)<\infty \forall x \in X$. Then

(C1) The pair $(A, U)$ satisfies property (S).

(C2) For any $\omega \subset X$ the representation $R^{\omega}$ is induced by a finite dimensional representation.

(C3) The pair $(A, U)$ satisfies property $(\mathrm{R})$.

Keeping in mind possible applications to harmonic analysis and representation theory we are mainly interested in the case of a Fourier algebra $A$.

Property (S) (Conjecture 1) was first studied by N. Varopoulos [24] for pairs $\left(A\left(\mathbf{R}^{n}\right), S O(n)\right)$, whose result was generalized by Y. Domar [3] and Kirsch and Müller [12]. The latter proved (S) for the algebra $\mathcal{A}\left(\mathbf{R}^{n}\right)$ and closed orbits $\omega$ of any Lie group $U$ acting on $\mathbf{R}^{n}$. This seems to be the most general known result, concerning $(\mathrm{S})$.

Nor do we know if the second conjecture is true in a situation essentially different from the above examples. One apparent difficulty is the very definition of the inducing representation $\tau$ and the space of $\tau$. The third example suggests $A / I(x)$ as a candidate, but Example 2 shows that the choice is wrong once $\omega$ is not a set of spectral synthesis for $A$.

In this paper we shall be concerned mainly with the third conjecture, i.e., property (R). It has some advantages compared with the first two. Namely (Proposition $2, \S 1$ ) it is stable under various "deformations" of the Banach space norm ("dense extensions" and "dense restrictions"). This allows, in particular, substitution of algebras with "good" synthesizibility properties like algebras of smooth functions, for "bad" algebras, e.g., group $L^{1}$-algebras, Fourier algebras, etc.

The main result of the paper is Theorem 1 , which establishes property $(\mathrm{R})$ for all pairs $\left(A_{\rho}(G), U\right)$ of the weighted Fourier algebra $A_{\rho}(G)$ on a LCA group $G$ with a subpolynomial weight $\rho$ on the dual group $A=\hat{G}$ (for definition, see $\S 1$ ) and a compact group $U$ acting by so-called affine transformations on $G$, i.e. combinations of automorphisms and translations.

Moreover, the multiplicities of $R^{\omega}$ are shown to admit the estimate (7),

$$
k\left(\pi ; R^{\omega}\right) \leq c(n, \rho) \cdot d(\pi) \quad \forall \pi \in \hat{U}
$$

with the constant $c(n, \rho)$ depending only on the dimension of the vector component $\mathbf{R}^{n}$ of $G$ and the weight $\rho$.

Theorem 1 has several applications $(\S 6)$. One of them concerns topologically irreducible representations of motion groups $G=A \rtimes U$ and maximal one-sided 
ideals of group algebras $L_{\rho}^{1}(G)$ on motion groups. The second one deals with primary ideals of invariant subalgebras $Z=L_{\rho}^{1}(A)^{U}$ on LCA groups $A$ and more general invariant subalgebras, which appear in the context of so-called $[F C]_{B}^{-}$groups (see $[\mathbf{8}, \mathbf{1 5}])$.

Let us sketch some basic ideas behind the proof of Theorem 1. For Euclidean (or more general compactly generated) groups $A$, i.e., Lie groups $G=\hat{A}$, the theorem was proved in $[\mathbf{1 0}]$. The argument based on Whitney's Theorem is essentially outlined in Example 3. To complete the details let us notice that for any polynomial weight $\rho$ on $A$, the Fourier algebra $A_{\rho}(G)$ (the Fourier transform of $L_{\rho}^{1}(A)$ ) contains a dense subalgebra $C_{0}^{m}(G)$ for sufficiently large $m$, depending on $\rho$ and $\operatorname{dim} G$. Using some easily verified properties of FM-representations (Proposition 2, §1) one can substitute $C_{0}^{m}(G)$ for $\mathcal{A}_{\rho}(G)$ and then proceed as in Example 3.

An opposite class of groups are LCA groups which contain an open compact subgroup (like $p$-adics $\mathbf{Q}_{p}^{n}$ ). Here the argument requires a different sort of ideas: a certain "reduction lemma" (cf. Lemma 4, §4), "inductive limits" (§1.2), and "synthesizibility of closed subgroups" (cf. Example 4 and Lemma 2, §3).

The general result (arbitrary LCA group) is a synthesis of both kinds of ideas. Namely, we study the structure of a LCA group $A$ (respectively dual group $G=\hat{A}$ ), upon which a compact group acts by affine transformations and "approximate" $G$ by Lie groups $G_{\alpha}$ (Lemma 4 ),

$$
G=\lim _{\alpha} \operatorname{pr} G_{\alpha}
$$

This implies that the representation $R^{\omega}=\lim _{\alpha}$ ind $R^{\omega_{\alpha}}$, where $R^{\omega_{\alpha}}$ is obtained from the action of $U$ on the Lie group $G_{\alpha}$. The problem is thus reduced to the "Lie-group" case. However at this point the main technical difficulty arises. We can no longer use Whitney's Theorem for $G_{\alpha}$ and substitute the algebra of smooth functions $C_{0}^{m}\left(G_{\alpha}\right)$ for the Fourier algebra $A_{\rho}\left(G_{\alpha}\right)$. Indeed, the degree of smoothness $m$, and therefore the multiplicities $k\left(\pi ; R^{\omega_{\alpha}}\right)$ (cf. Example 3) depend on $\operatorname{dim} G_{\alpha}$ and go to $\infty$ as $\operatorname{dim} G_{\alpha} \rightarrow \infty$. So there is no way to control the multiplicity of $\pi$ in $R^{\omega}$. Now instead of the algebra $C_{0}^{m}(G)$ with "nice" synthesizibility properties we have to consider the algebra $C_{0}^{m}\left(\mathbf{R}^{n} ; B\right)$ of all $m$-smooth $B$-valued functions on $\mathbf{R}^{n}$, where $B=A_{\rho}(X)$ is the Fourier algebra on the compact component $X$ of $G\left(G=\mathbf{R}^{n} \times X\right)$.

A crucial step in the proof of Theorem 1 is Lemma 3, which gives a Whitney-type characterization of minimal ideals $I(\omega)$ of the algebra $C_{0}^{m}\left(\mathbf{R}^{n} ; B\right), \omega \subset \mathbf{R}^{n} \times X$.

Let us notice that Whitney's Theorem extends to vector-valued functions with values in a-finite dimensional space B (see [16, Chapter 2]). However, the "Banach" verson is not known in general and probably not true.

In $\S 5$ we prove this result for a special class of submodules $\mathcal{M} \subset C_{0}^{m}\left(\mathbf{R}^{n} ; B\right)$ (essentially, for $\mathcal{M}=I(\omega)$ ). The lengthy argument of $\S 5$ exploits a variety of techniques: "jets", "Whitney functions", "vector bundles", "induced representations", etc. Some of them are known in the classical Whitney's theory (cf. $[\mathbf{1 6}, \mathbf{2 6}]$ ), some others are new in this context.

Let us briefly describe the organization of the paper. $\S 1$ contains preliminary definitions and results, such as weights, Beurling (weighted) group algebras and Fourier algebras. We also discuss two important classes of representations of compact groups, induced representations and finitely multiplied (FM) representations. 
Then ( $§ 2)$ we formulate the main Theorem 1.

Most of the paper ( $\S 3-5)$ deals with the proof of Theorem 1 . In $\S 3$ we prove it for compactly generated groups $A$, respectively Lie groups $G=\hat{A}$.

Then (§4) we study the action of a compact group $U$ on a LCA group $A$, and complete the proof of Theorem 1 via certain limit procedures. We also indicate an extension of Theorem 1 to the case when a compact group $U$ acts by automorphisms of the Fourier algebra $A(G)$.

An important Lemma 3 (Whitney-type characterization of minimal ideals $I(\omega) \subset$ $\left.C_{0}^{m}\left(\mathbf{R}^{n} ; \beta\right)\right)$ is proved in $\S 5$, some results of which are of independent interest.

In the last section $(\S 6)$ we give applications of Theorem 1 to Banach irreducible representations of motions groups (Burnside type theorem) and primary ideals of invariant subalgebras.

Two appendices $A$ and $B$ include some technical results needed in $\S 5$.

We would like to thank a referee for a number of helpful suggestions.

\section{Preliminaries.}

1.1. Weights, Beurling and Fourier algebras, finiteness conditions. Let $G$ be a locally compact group. By a weight on $G$ we mean a measurable locally bounded submultiplicative function $\rho: G \rightarrow[1, \infty)$, i.e.,

$$
\rho(x \cdot y) \leq \rho(x) \rho(y) \quad \forall x, y \in G .
$$

We also assume that $\rho$ is symmetric $\rho(x)=\rho\left(x^{-1}\right)(x \in G)$.

The space $L_{\rho}^{1}(G)$ of all $\rho$-summable functions $f$ on $G$ with norm

$$
\|f\|=\int_{G}|f(x)| \rho(x) d x
$$

( $d x$ is a Haar measure on $G$ ) and convolution as multiplication, forms a Banach algebra called the weighted (or Beurling) group algebra. The usual $L^{1}$-group algebras $L^{1}(G)$ correspond to the trivial weight $\rho, \rho(x)=1 \forall x \in G$.

These algebras naturally appear in Banach representation theory. Let $x \mapsto$ $T_{x}(x \in G)$ be a strongly continuous Banach representation of $G$. Then the function $\rho(x)=\left\|T_{x}\right\|$ is obviously a semicontinuous weight on $G$. The representation $T$ extends to a representation of the group algebra $L_{\rho}^{\mathbf{1}}(G)$,

$$
f \mapsto T_{f}=\int_{G} f(x) T_{x} d x .
$$

Moreover, there is a 1-1 correspondence between nondegenerate representations $T$ of the Beurling algebra $L_{\rho}^{1}(G)$ and those representations $T$ of $G$, whose growth is dominated by $\rho$, i.e., $\left\|T_{x}\right\| \leq c \cdot \rho(x)(x \in G)$.

Given a pair of weights $\rho$ and $\rho^{\prime}$ on $G$ we say that $\rho^{\prime}$ is stronger than $\rho$, if $L_{\rho^{\prime}}^{1}(G) \subset L_{\rho}^{1}(G)$, or equivalently $\rho(g) \leq c \cdot \rho^{\prime}(g)$ a.e. on $G$ for some constant $c>0$. If $L_{\rho^{\prime}}^{1}(G)=L_{\rho}^{1}(G)$ we call $\rho$ and $\rho^{\prime}$ equivalent.

Further on we shall often deal with a situation when a compact group $U$ acts on a locally compact group $G$. The typical actions will be the action of $U$ by automorphisms of $G$ or the action of a subgroup $U \subset G$ by left (right) translations on $G$.

In both cases we can assume the weight $\rho$ on $G$ to be $U$-invariant, i.e.;

(I) $\rho\left(g^{u}\right)=\rho(g) \forall g \in G, u \in U$, when $U$ acts by automorphisms. 
(II) $\rho(u g v)=\rho(g) \forall g \in G, u, v \in U$, when $U$ acts by translations.

In the second case any weight $\rho$ on $G$ is equivalent to a $U$-biinvariant one, while in the first case this condition is necessary and sufficient in order that the action of $U$ on $G$ gives rise to a Banach representation of $U$ on the space $L_{\rho}^{1}(G)$.

Let $A$ be a locally compact abelian group with the dual group $G=\hat{A}$. A sufficient condition on $\rho$ for the group algebra $L_{\rho}^{1}(A)$ to be regular and Wiener is the nonquasianalyticity condition [2]

$$
\sum_{-\infty}^{\infty} \frac{\log \rho(n a)}{1+n^{2}}<\infty \quad \forall a \in A .
$$

It will be assumed elsewhere in the sequel.

We denote by $\mathcal{\exists}: f(a) \mapsto \hat{f}(x)$ the Fourier transform on $A$,

$$
\mathcal{F}: f \mapsto \hat{f}(x)=\int_{A} f(a)\langle\overline{x, a}\rangle d a \quad(x \in G)
$$

The map $₹$ takes $L_{\rho}^{1}(A)$ into the algebra $C_{\infty}(G)$ of all continuous functions vanishing at $\infty$, and the image $\mathcal{F} L_{\rho}^{1}(A)=A_{\rho}(G)$, equipped with the norm of $L_{\rho}^{1}(A)$, is called a weighted Fourier algebra on $G$.

We discuss some "finiteness" conditions on the weight $\rho$, which assure property (F) of the Fourier algebra. The translations $W_{y}: \hat{f}(x) \mapsto \hat{f}(x+y)$ form a transitive family of isometric automorphisms of the Fourier algebra $A_{\rho}(G)$. In particular, all quotients $\tilde{A}(x)=A_{\rho}(G) / I(x)$ are isomorphic (here $I(x)$ is the minimal primary ideal of $\mathcal{A}_{\rho}(G)$ at $\left.\{x\}\right)$ and the dimensions $m(x)=\operatorname{dim} \tilde{A}(x)$ are equal for all $x \in G$,

$$
m(x)=m(0)=m^{\prime}(\rho) \text {. }
$$

We shall call a weight $\rho$ finite if the number $m^{\prime}(\rho)<\infty$. Given an open subgroup $B \subset A$, we denote by $\rho_{B}$ the restriction of $\rho$ on $B$ and by $m(\rho, B)$ the number $m^{\prime}\left(\rho_{B}\right)$. The weight $\rho$ on $A$ is called uniformly finite, if the number

$$
m(\rho)=\sup \{m(\rho, B) \mid B \subset A\}<\infty .
$$

The sup is taken over all compactly generated open subgroups $B \subset A$.

A simple "inductive limit" argument (cf. Proposition 3) shows that each uniformly finite weight is finite and $m^{\prime}(\rho) \leq m(\rho)$. It seems plausible that both conditions are equivalent and $m^{\prime}(\rho)=m(\rho)$ but we cannot prove this in general. The trivial weight $\rho(a) \equiv \mathbf{1}$ is uniformly finite by Ditkin-Helson's Theorem (see $[\mathbf{1 1}$, Chapter 10]). Here $m^{\prime}(\rho)=m(\rho)=1$.

In the special case $A=\mathbf{R}^{n}, \mathbf{Z}^{k}(k<\infty)$ or any compactly generated group $A$ the polynomial growth condition suffices for uniform finiteness,

$$
\forall a \in A, \quad \rho(n a)=O\left(|n|^{\alpha}\right) \quad \text { as } n \rightarrow \infty \text { for some } \alpha=\alpha(a)
$$

but in general neither condition (1.1.6) or (1.1.7) implies the other. So we must often impose both of them in order to get a required result.

Precisely, by the well-known structure theorem [19] any LCA group $A$ splits into the direct sum of a vector group $L \cong \mathbf{R}^{n}$ and a group $D$ which contains an open compact subgroup $K \subset D$. Given a weight $\rho$ on $A$ we denote by $\rho^{\prime}$ and $\rho^{\prime \prime}$ its restrictions on $L$ and $D$ respectively. 
DEFINITION 1. The weight $\rho$ is called subpolynomial if $\rho^{\prime}=\rho \mid L$ is polynomial and $\rho^{\prime \prime}=\rho \mid D$ is uniformly finite.

One can show that this condition has an equivalent form: the restriction $\rho^{\prime}=$ $\rho \mid L$ is polynomial and the weight $\rho$ on the whole group $A$ is uniformly finite. In particular, the subpolynomial growth condition is independent of a particular choice of components $L$ and $D$ in $A$ (they are not uniquely determined, in general).

With any subpolynomial weight $\rho$ we associate two invariants: $m\left(\rho^{\prime \prime}\right)$, the degree of finiteness of $\rho^{\prime \prime}(1.1 .6)$ and

$$
\alpha\left(\rho^{\prime}\right)=\inf \left\{\alpha \mid \rho^{\prime}(x)=O\left(|x|^{\alpha}\right) \text { as } x \rightarrow \infty\left(x \in \mathbf{R}^{n}\right)\right\},
$$

the exponent of $\rho^{\prime}$.

Let us remark that finiteness of the weight (i.e. property $(\mathrm{F})$ of the algebra $A_{\rho}(G)$ ) is generically (in the sense of the action of $U$ on $G$ ) a "necessary" condition in order to have results of the type of Theorem 1 . We require a close but little stronger condition ("uniform finiteness" and "polynomial growth") for technical reasons.

1.2. Finitely multiplied representations. We shall introduce an important class of representations of compact groups needed further on.

Let $U$ be a compact group, $\hat{U}$ its dual object, the set of all equivalence classes of irreducible representations of $U$. For an irreducible representation $\pi \in \hat{U}$ we denote its space by $E_{\pi}$, its degree by $d(\pi)=\operatorname{dim} E_{\pi}$ and its character by $\chi_{\pi}=\operatorname{tr} \pi$.

Let $u \mapsto T_{u}$ be a Banach representation of $U$ on the space $E$. As usual, we define a primary projection

$$
P_{\pi}=d(\pi) \int \bar{\chi}_{\pi}(u) T_{u} d u
$$

and a primary (isotypical) subspace $E(\pi)=P_{\pi} E$. The subspace $E(\pi)$ is the maximal invariant subspace of $T$ such that the restriction $T \mid E(\pi)$ is equivalent to a multiple of $\pi$. Let us denote by $k_{T}(\pi)$ the multiplicity of $\pi$ in $T$,

$$
k_{T}(\pi)=\operatorname{dim} E(\pi) / d(\pi) .
$$

A representation $T$ is called finitely multiplied (or FM-representation) if $k_{T}(\pi)<$ $\infty, \pi \in \hat{U}$.

The well-known examples of FM-representations are induced representations $T=\operatorname{ind}_{V}^{U}(\tau)$, where $V \subset U$ is a closed subgroup and $\tau$ is a finite dimensional representation of $V$.

Throughout this paper we shall often use a notion of induced representation in Banach space setting. So let us briefly discuss some basic definitions and results. We refer to $[\mathbf{5}, \mathbf{1 7}, \mathbf{2 1}]$ for more details.

Induced representations are naturally defined in terms of Banach vector bundles (or continuous fields of Banach spaces) (see [5]). By the latter we mean triples $(\mathcal{E}, p, X)$ of topological spaces $\mathcal{E}$ and $X$ (base) and a continuous projection $p: \mathcal{E} \rightarrow$ $X$, such that all fibres $E_{x}=p^{-1}(x)(x \in X)$ are Banach spaces. The triple $(\mathcal{E}, p, X)$ satisfies some natural continuity assumptions (see [5, Chapter II]), in particular there is a notion of a continuous cross-section $f: X \rightarrow \mathcal{E}\left(f(x) \in E_{x} \forall x \in X\right)$ and the space of all continuous sections has "enough" elements [5, Appendix].

One naturally defines morphisms, e.g., homomorphism, isomorphisms, etc. of Banach bundles. In particular, the group $U$ acts by automorphisms of $(\mathcal{E}, p, X)$ if 
to each element $u \in U$ there corresponds a pair of homeomorphisms $u: X \rightarrow X$ and $\tilde{u}: \mathcal{E} \rightarrow \mathcal{E}$ such that:

(1) $p\left(\xi^{\tilde{u}}\right)=p(\xi)^{u} ; \forall x \in X ; \xi \in E_{x} ; u \in U$.

(2) The map $\tau(x, u): E_{x} \rightarrow E_{x^{u}}\left(\tau(x, u) \xi=\xi^{\tilde{u}}\right)$ is a linear isomorphism of Banach spaces $E_{x}$ and $E_{x^{u}}$.

(3) For any $\xi \in \mathcal{E}$, the function $u \rightarrow \xi^{\tilde{u}}$, from $U$ to $\mathcal{E}$ is continuous.

The action of $U$ on the bundle $(\mathcal{E}, p, X)$ induces a representation of $U$ on the space of all continuous sections $\Gamma(X, \mathcal{E})$,

$$
\left(T_{u} f\right)(x)=\tau(x, u)^{-1} f\left(x^{u}\right), \quad f \in \Gamma(X, \mathcal{E}), u \in U .
$$

We shall call $(\mathcal{E}, p, X)$ a homogeneous Banach bundle if the base space $X$ is a homogeneous $U$-space, $X \cong V \backslash U$.

One can show (cf. $[\mathbf{5}, \mathbf{2 1}])$ that a homogeneous Banach bundle $(\mathcal{E}, p, X)$, and consequently the representation $T$ in (1.2.2), is uniquely (up to equivalence) determined by a single fibre $E_{x}(x \in X)$ and the equivalence class of a representation $v \mapsto \tau(x, v)$ of a stabilizer $V=U_{x}$ on the space $E_{x}$. The corresponding representation $T$ of $U$ is called induced and denoted by $\operatorname{Ind}_{V}^{U}(\tau)(\tau=\tau(x, \cdot))$. More general induced representations, in particular, representations in spaces of measurable sections $\left(L^{p}(X, \mathcal{E})\right.$, etc. $)$ were studied in $[\mathbf{5}, \mathbf{1 7}, \mathbf{2 1}]$.

Here we shall be concerned only with induced representations of compact groups in spaces of continuous sections and later on $(\S 5)$ in spaces of smooth sections.

A decomposition of an induced representation into the sum of isotypical components is described by the well-known Frobenius reciprocity theorem (see $[\mathbf{1 7}$, 21]).

Given a pair of Banach representations $T$ and $S$ of a compact group $U$ we denote by $\operatorname{Hom}_{U}(T ; S)$ the space of all bounded intertwining operators and by $k(T ; S)$ the intertwining number,

$$
k(T ; S)=\operatorname{dim} \operatorname{Hom}_{U}(T ; S)=\sum_{\pi \in \hat{U}} k_{T}(\pi) \cdot k_{S}(\pi) .
$$

Proposition 1 (FrobeniUs RECIPROCITY THEOREM). If $T=\operatorname{Ind}_{V}^{U}(\tau)$ is induced and $S$ is a Banach representation of a compact group $U$, then the spaces $\operatorname{Hom}_{U}\left(S ; \operatorname{Ind}_{V}^{U}(\tau)\right)$ and $\operatorname{Hom}_{V}(S \mid V ; \tau)$ are naturally isomorphic. In particular, if $S=\pi \in \hat{U}$ is irreducible, the multiplicity

$$
k_{T}(\pi)=k(\pi ; T)=k(\pi \mid V ; \tau) .
$$

Since the right-hand part of (1.2.4) is finite for a finite dimensional $\tau$, the induced representation $T=\operatorname{Ind}_{V}^{U}(\tau)$ is finitely multiplied.

Propostion 1 is well known in the case of measurable sections $[\mathbf{1 7}, \mathbf{2 1}]$. The "continuous" version goes along the same lines and the proof becomes even easier owing to continuity. We omit details.

Let us mention some properties of FM-representations needed further on.

PROPOSITION 2. Let $T, S$ be a pair of Banach representations and $W \in$ $\operatorname{Hom}_{U}(S, T)$ be an intertwining operator.

(a) If the range of $W$ is dense, then the finite multiplicity of $S$ implies the finite multiplicity of $T$ and $k_{T}(\pi) \leq k_{S}(\pi) \forall \pi \in \hat{U}$.

(b) If the kernel of $W$ is zero, then the inverse is true and $k_{S}(\pi) \leq k_{T}(\pi)$. 
If in particular both assumptions (a) and (b) hold, i.e., $T$ and $S$ are Naimarkequivalent, then $k_{S}(\pi)=k_{T}(\pi), \pi \in \hat{U}$.

(c) If $S$ is an FM-representation of a closed subgroup $V \subset U$ and $T=\operatorname{Ind}_{V}^{U}(S)$, then $T$ is an FM-representation of $U$.

(d) The tensor (Kronecker) product of an FM-representation $T$ and a finite dimensional representation $\sigma, T \otimes \sigma$, is also $F M$.

The proof of this statement is easy and can be found in [10, Lemma 1].

The following proposition concerns inductive limits of FM-representations. Let $\Sigma=\{\alpha, \beta, \ldots\}$ be a partially ordered set and $\left\{E_{\alpha}\right\}_{\alpha \in \Sigma}$ be an inductive family of Banach $U$-modules. This means that we are given a representation $T^{\alpha}$ of $U$ on each $E_{\alpha}$ and a family of intertwining operators $W_{\alpha}^{\beta} \in \operatorname{Hom}_{U}\left(T^{\alpha} ; T^{\beta}\right), \alpha<\beta$, such that

$$
W_{\beta}^{\gamma} \cdot W_{\alpha}^{\beta}=W_{\alpha}^{\gamma} \quad \forall \alpha<\beta<\gamma .
$$

We shall call a Banach $U$-module $E$ an inductive limit of the family $\left\{E_{\alpha}\right\}_{\alpha \in \Sigma}$, if there exists a family of intertwining operators $\left\{W_{\alpha} \in \operatorname{Hom}_{U}\left(E_{\alpha}, E\right)\right\}_{\alpha}$, whose ranges span a dense subspace in $E$, such that $W_{\alpha}=W_{\beta} \cdot W_{\alpha}^{\beta} \forall \alpha<\beta$.

PROPOSITION 3. If all representations $\left\{T^{\alpha}\right\}_{\alpha \in \Sigma}$ are $F M$ and the multiplicities $\left\{k\left(\pi ; T^{\alpha}\right)\right\}_{\alpha}$ are uniformly bounded, i.e.,

$$
k\left(\pi ; T^{\alpha}\right) \leq m(\pi) \quad \forall \pi \in \hat{U}, \alpha \in \Sigma,
$$

then the inductive limit $T=\lim _{\alpha}$ ind $T_{\alpha}$ is also $F M$, and $k(\pi ; T) \leq m(\pi) \forall \pi \in \hat{U}$.

PROOF. Let us consider $\pi$-primary components $E(\pi)$ and $E_{\alpha}(\pi)$ of the representations $T$ and $T^{\alpha}$. Obviously, $W_{\alpha}\left(E_{\alpha}(\pi)\right) \subseteq E(\pi)$ and the inductive family of finite dimensional subspaces $\left\{F_{\alpha}=W_{\alpha}\left(E_{\alpha}(\pi)\right)\right\}_{\alpha}\left(\operatorname{dim} F_{\alpha} \leq \operatorname{dim} E_{\alpha}(\pi)\right)$ span a dense subspace in $E(\pi)$. But the dimensions of $\left\{F_{\alpha}\right\}_{\alpha}$ are uniformly bounded.

$$
\operatorname{dim} F_{\alpha} \leq \operatorname{dim} E_{\alpha}(\pi)=d(\pi) \cdot k\left(\pi ; T^{\alpha}\right) \leq d(\pi) \cdot m(\pi) .
$$

Hence $\operatorname{dim} E(\pi) \leq d(\pi) \cdot m(\pi)$, i.e., $k(\pi ; T) \leq m(\pi) \forall \pi \in \hat{U}$. Q.E.D.

2. The main theorem. Let $G$ be a LCA group with dual group $\hat{G}$ and let $\rho$ be a subpolynomial weight on $\hat{G}$. We decompose $\hat{G}$ into the sum $\mathbf{R}^{n} \oplus D$, where $D$ has a compact-open subgroup, and write $\rho^{\prime}$ and $\rho^{\prime \prime}$ for restrictions $\rho^{\prime}=\rho \mid \mathbf{R}^{n}$ and $\rho^{\prime \prime}=\rho \mid D$. By definition, $\rho^{\prime}$ is polynomial, i.e.,

$$
\rho^{\prime}(x)=O\left(|x|^{\alpha}\right) \quad \text { as } x \rightarrow \infty \text { for some } \alpha \geq 0
$$

while $\rho^{\prime \prime}$ is uniformly finite. As above we denote by $m\left(\rho^{\prime \prime}\right)$ the degree of finiteness of $\rho^{\prime \prime}$ and by $\alpha\left(\rho^{\prime}\right)$ the exponent of $\rho^{\prime}$, i.e., the infimum of all $\alpha$ satisfying (2.1.1).

We consider the action of a compact group $U$ by affine transformations on $G$. Recall that an affine transformation is a continuous map $\tilde{u}: G \rightarrow G$, which satisfies

$$
(a+b-c)^{\tilde{u}}=a^{\tilde{u}}+b^{\tilde{u}}-c^{\tilde{u}} \quad \forall a, b, c \in G .
$$

So $\tilde{u}$ is uniquely determined by a pair $(u, l)$ of an automorphism $u \in \operatorname{Aut}(G)$ and a translation with an element $l \in G$,

$$
a^{\tilde{u}}=a^{u}+l \quad(a \in G) .
$$

The group of affine transformations $\operatorname{Aff}(G)$ is isomorphic to a semidirect product $G \rtimes \operatorname{Aut}(G)$. Hence an affine action of $U$ on $G$ consists of a pair of an automorphic 
action $u: a \rightarrow a^{u}(a \in G, u \in U)$ and a cocycle $l(u)$, i.e. a continuous function $l: U \rightarrow G$ s.t.

$$
l\left(u_{1} u_{2}\right)=l\left(u_{1}\right)^{u_{2}}+l\left(u_{2}\right) \quad \forall u_{1}, u_{2} \in U .
$$

Obviously the action of $U$ by automorphisms of $G$ as well as the action of a subgroup $Y \subset G$ by translations on $G$ are special cases of affine actions. Another important example arises as follows.

EXAMPLE 5. Let $X$ be a subgroup of a LCA group $B$ and let the group $U$ act by automorphisms of $B$, which leave $X$ invariant. Then $U$ acts also on the factor group $\dot{B}=B / X$. Let $\dot{b} \in B$ be a $U$-stable coset, i.e., $\dot{b}^{u}=\dot{b}(u \in U)$. We choose an element $b$ in the coset $\dot{b}=b+X$ and define a cocycle $l: U \rightarrow X$, to be

$$
l(u)=b^{u}-b \quad(u \in U) .
$$

Obviously $l$ is continuous and satisfies equation (2.1.3). The automorphic action of $U$ on the coset $\dot{b}=b+X$ and its cocyclic action on $X$ are naturally related. Indeed, the translation $W: x \rightarrow x+b(x \in X)$ from $X$ onto $\dot{b}$ permutes these actions,

$$
W\left(x^{\tilde{u}}\right)=\left(x^{u}+b^{u}-b\right)+b=(x+b)^{u}=[W(x)]^{u} .
$$

Notice that each affine transformation defines an automorphism of the Fourier algebra $\mathcal{A}_{\rho}(G)$,

$$
\tilde{u}: \hat{f}(x) \rightarrow \hat{f}\left(x^{\tilde{u}}\right) \quad\left(u \in U ; \hat{f} \in \mathcal{A}_{\rho}(G)\right) .
$$

The main result of this paper is the following

THEOREM 1. Let a compact group $U$ act by affine transformations on a LCA group $G$ and let $A_{\rho}(G)$ be a Fourier algebra on $G$ with a subpolynomial weight $\rho$ on $A=\hat{G}$. Then for any orbit $\omega \subset G$ the representation $R^{\omega}$ is finitely multiplied. Moreover, the multiplicities $k\left(\pi ; R^{\omega}\right)$ are estimated as

$$
k\left(\pi ; R^{\omega}\right) \leq c(n, \rho) d(\pi), \quad \forall \pi \in \hat{U}
$$

with the constant $c(n, \rho)=m\left(\rho^{\prime \prime}\right)\left(\begin{array}{c}m+n \\ m\end{array}\right)$, where $m\left(\rho^{\prime \prime}\right)$ is the degree of finiteness of $\rho^{\prime \prime}, n$ is the dimension of the vector component $\mathbf{R}^{n}$ of $A$ and $m=[n+1+\alpha]$ with $\alpha=\alpha\left(\rho^{\prime}\right)$, the exponent of $\rho^{\prime}$.

Obviously $c(n, \rho)$ depends only on $n$ and $\rho$.

The appearance of the number $m=[n+1+\alpha]$ and the binomial coefficient $\left(\begin{array}{c}m+n \\ n\end{array}\right)$ in estimate (2.1.5) will become clear later on. Their "geometrical" meaning is the following: $m$ is the smallest integer such that the space $C_{0}^{m}\left(\mathbf{R}^{n}\right)$ is contained in the Fourier algebra $A_{\rho^{\prime}}\left(\mathbf{R}^{n}\right)$, while $\left(\begin{array}{c}m+n \\ m\end{array}\right)=d\left(\tau_{m}\right)$ is the degree of the representation $\tau_{m}$ constructed in Example 3 of the Introduction.

\section{Compactly generated-Lie group case.}

3.1. We start the proof of Theorem 1 with the case of a compactly generated group $A$, respectively Lie group $G=\hat{A}[\mathbf{1 9}]$. By Lie group we mean a (not necessarily connected) locally compact group $B$, whose identity component $B_{0}$ is a connected Lie group and such that the factor group $B / B_{0}$ is discrete.

In this section we shall prove the following version of Theorem 1. 
LEMMA 1. Let a compact group $U$ act by affine transformations on an abelian Lie group $G$. If $A_{\rho}(G)$ is a Fourier algebra on $G$ with a subpolynomial weight $\rho$ on $A=\hat{G}$, then for any orbit $\omega \subset G$ the representation $R^{\omega}$ is finitely multiplied and its multiplicities are estimated as

$$
k\left(\pi ; R^{\omega}\right) \leq c(n, \rho) d(\pi) \quad \forall \pi \in \hat{U},
$$

with the constant $c(n, \rho)$ of Theorem 1 .

For convenience we divide the proof of Lemma 1 into 4 steps. We start with the simplest case of affine action, i.e., the action of a closed subgroup $Y$ of a compact group $X$ by translations on $X$ (Lemma 2). Here $A=A_{\rho}(X), \omega=Y+x_{0}$ is a coset, and the multiplicities

$$
k\left(\mu ; R^{\omega}\right) \leq m(\rho) \quad(\text { the degree of finiteness of } \rho) .
$$

Next (3.3) we consider a Lie group $\mathbf{R}^{n} \oplus X$ with the following actions of $U$ on components: affine action on $\mathbf{R}^{n}$ and translations with elements of a subgroup $Y=l(U)$ on $X$. Here $l: U \rightarrow X$ is a group homomorphism. We show that the Fourier algebra

$$
A_{\rho}\left(\mathbf{R}^{n} \oplus X\right)=A_{\rho^{\prime}}\left(\mathbf{R}^{n}\right) \hat{\oplus} A_{\rho^{\prime \prime}}(X)
$$

can be replaced by a dense subalgebra

$$
A=C_{0}^{m}\left(\mathbf{R}^{n} ; B\right), \quad B=A_{\rho^{\prime \prime}}(X),
$$

of all $m$-smooth compactly supported $B$-valued functions on $\mathbf{R}^{n}$.

Then we formulate an important Lemma 3 , a Whitney-type characterization of minimal ideals $I(\omega) \subset \mathcal{A}=C_{0}^{m}\left(\mathbf{R}^{n} ; B\right)$ with hulls $\omega \subset \mathbf{R}^{n} \oplus X$, whose proof is given in $\S 5$.

Lemma 3 enables us to embed the representation $R^{\omega}$ on $A / I(\omega)(A=$ $\left.C_{0}^{m}\left(\mathbf{R}^{n} ; B\right)\right)$ into a certain induced representation $T$, composed of the representation $\tau_{m}$ of Example 3 of the Introduction and the representation of Lemma 2. The multiplicities of the latter are estimated as $k(\pi ; T) \leq c(n, \rho) d(\pi)$ with the constant $c(n, \rho)$ of Theorem 1 .

In the next two steps we use structure results of $\S 4$ to reduce a general affine action on the Lie group $G=\mathbf{R}^{n} \oplus X$ (X compact) to the action of Lemma 3 (3.4). Finally in (3.5) we proceed to a general Lie group $G$.

3.2. LEMMA 2. Let $\rho$ be a uniformly finite weight on a discrete abelian group $A$ dual to a compact group $X$. If $Y \subset X$ is a closed subgroup, then the representation $R^{Y}$ of $Y$ on the quotient space $A_{\rho}(X) / I(Y)$ is FM. Moreover, we get the estimate of multiplicities

$$
k\left(\lambda, R^{Y}\right) \leq m(\rho) \quad \forall \lambda \in \hat{Y} .
$$

PROOF. Let us notice that for the trivial weight $\rho$, i.e. the Fourier algebra $A(X)$, Lemma 3 follows from the well-known result [20], by H. Reiter (see Example 4). Indeed, according to [20], any closed subgroup $Y$ of a LCA group $X$ is a set of synthesis, i.e., $J(Y)=I(Y)$. So the quotient algebra is isomorphic to the Fourier algebra $A(Y)$ and the representation $R^{Y}$ is equivalent to the regular representation of $Y$ on the space $A(Y)$. The regular representation $R$ of a compact abelian group $Y$ on a translation invariant space $A(Y)$ is well known to decompose into the direct 
sum of characters, each of them occurring in $R$ with multiplicity 1 . So the equality in (3.2.1) holds with $m=1$. But $m=1$ is precisely the degree of finiteness of the trivial weight $\rho$ by Helson-Ditkin's Theorem [11, Chapter 10].

One can expect that a similar result holds for weighted Fourier algebras $A_{\rho}(X)$. Namely, the representation $R^{Y}$ of $Y$ in the quotient space $A_{\rho}(Y) / I(Y)$ is equivalent to a multiple of the regular representation $R$ on $A_{\rho}(X) / I(Y) \cong A_{\rho}(Y)$, i.e.,

$$
R^{Y} \sim R \otimes m^{\prime}
$$

for some $m^{\prime}$ depending on $\rho$ and $Y$. Of course, this would immediately imply (3.2.1).

Actually we shall prove somewhat less. We shall prove the factorization formula (3.2.2) for another Fourier algebra $A_{1}=A_{\rho_{1}}\left(X_{1}\right)$ on a different group $X_{1}$, which is densely embedded into $A=A_{\rho}(X)$. Then the "density" argument of Proposition 2 will give us the estimate (3.2.1) for the Fourier algebra $A=A_{\rho}(X)$ itself.

Let $X$ be a compact abelian group, $Y \subset X$ be a closed subgroup and $Z=X / Y$ be the factor group. We denote by $A=\hat{X}, B=\hat{Y}$ and $C=\hat{Z}$ their dual (discrete) groups, $A \supset C$ and $A / C \cong B$.

Given a weight $\rho$ on $A$ let us define the weights $\rho^{\prime}$ and $\rho^{\prime \prime}$ on $C$ and $B$ as $\rho^{\prime}=\rho \mid C$ and $\rho^{\prime \prime}(b)=\inf \{\rho(a+c) \mid c \in C\}(b=a+C \in B)$.

We shall consider a discrete abelian group $A_{1}=B \oplus C$ and the weight $\rho_{1}=\rho^{\prime} \times \rho^{\prime \prime}$ on $A_{1}, \rho_{1}(b, c)=\rho^{\prime \prime}(b) \cdot \rho^{\prime}(c)(b \in B, c \in C)$.

The group algebra $L_{\rho_{1}}^{1}\left(A_{1}\right)$ factors into the product $L_{\rho^{\prime}}^{1}(C) \otimes L_{\rho^{\prime \prime}}^{1}(B)$ with the strongest (projective) cross-norm.

Correspondingly the Fourier algebra $B_{1}$ of $L_{\rho_{1}}^{1}\left(A_{1}\right)$ factors as

$$
B_{1}=A_{\rho^{\prime}}(Z) \hat{\otimes} A_{\rho^{\prime \prime}}(Y) \text {. }
$$

The group $Y$ acts by translations on both Fourier algebras $B_{1}=A_{\rho_{1}}(Y \oplus Z)$ and $B=A_{\rho}(X)$ :

$$
y_{0} \mapsto R_{y_{0}}: \hat{f}(y, z) \rightarrow \hat{f}\left(y+y_{0}, z\right) \quad\left(y, y_{0} \in Y, z \in Z\right)
$$

Correspondingly it acts by multiplication with a character $\left\langle y_{0}, b\right\rangle$ on the group algebras $L_{\rho_{1}}^{1}(B \oplus C)$ and $L_{\rho}^{1}(A)$ :

$$
f(b, c) \mapsto\left\langle y_{0}, b\right\rangle \cdot f(b, c), \quad f \in L_{\rho_{1}}^{1}(B \oplus C) .
$$

The subalgebra $A_{\rho^{\prime}}(Z)$ acts on the Fourier algebras $B_{1}$ and $B$ by multiplication,

$$
\hat{\varphi}: \hat{f} \mapsto \hat{\varphi}(z) \cdot \hat{f}(y, z), \quad \hat{f} \in B_{1} \text { or } \hat{B}, \hat{\varphi} \in A_{\rho^{\prime}}(Z) .
$$

For any $\varepsilon>0$ we can choose a system of coset representatives $\left\{a_{b} \mid b \in B\right\} \subset A$ with the property

$$
\rho\left(a_{b}\right) \leq \rho^{\prime \prime}(b)+\varepsilon \quad \forall b \in B .
$$

Given such a system we define a linear operator $W: L_{\rho_{1}}^{1}\left(A_{1}\right) \rightarrow L_{\rho}^{1}(A)$ to be $(W f)(a)=f(b ; c), f \in L_{\rho_{1}}^{1}\left(A_{1}\right)$, where $a=a_{b}+c(b \in B, c \in C)$ is a unique decomposition of an element $a \in A$.

The operator $W$ is easily seen to have the following properties.

(a) $\|W f\|_{\rho} \leq(1+\varepsilon)\|f\|_{\rho_{1}}$.

(b) The range of $W$ is dense in $L_{\rho}^{1}(A)$. 
(c) $W: B_{1} \rightarrow B$ commutes with the actions (3.2.3) and (3.2.5) of the subgroup $Y$ and the subalgebra $A_{\rho^{\prime}}(Z)$ on $B_{1}$ and $B$.

Now let us consider the minimal ideal $I(Y)$ of the algebra $B_{1}=A_{\rho^{\prime \prime}}(Y) \hat{\otimes} A_{\rho^{\prime}}(Z)$. We claim that

$$
I(Y)=A_{\rho^{\prime \prime}}(Y) \hat{\otimes} I(0)
$$

$I(0)$ being the minimal primary ideal of $\mathcal{A}_{\rho^{\prime}}(Z)$ at $\{0\}$. Indeed, the right-hand side of (3.2.6) is obviously included in $I(Y)$. Conversely, if a function $\hat{f} \in B_{1}$ vanishes on a neighborhood of $Y$, there exists $\hat{\varphi} \in \mathcal{A}_{\rho^{\prime}}(Z)$ vanishing on a neighborhood of $0 \in Z$ such that $\hat{\varphi} \hat{f}=\hat{f}(\hat{\varphi}$ considered as a function on $Y \oplus Z)$. By the definition of tensor products,

$$
\hat{f}=\lim _{k \rightarrow \infty}\left(\sum_{j=1}^{k} \hat{f}_{j} \otimes \hat{g}_{j}\right) \quad\left(\hat{f}_{j} \in A_{\rho^{\prime \prime}}(Y), \hat{g}_{j} \in A_{\rho^{\prime}}(Z)\right) .
$$

Multiplying both sides of (3.2.7) by $\hat{\varphi}$ we get $\hat{\varphi} f=\lim \sum\left(\hat{f}_{j} \otimes \hat{\varphi} \hat{g}_{j}\right)$. Of course each element $\hat{f}_{j} \otimes \hat{\varphi} \hat{g}_{j} \in A_{\rho^{\prime \prime}}(Y) \hat{\otimes} I(0)$. So both ideals in (3.2.6) are equal. In particular, the quotient algebra $\tilde{B}_{1}(Y)=B_{1} / I(Y)$ is isomorphic to the product

$$
A_{\rho^{\prime \prime}}(Y) \hat{\otimes}\left(\mathcal{A}_{\rho^{\prime}}(Z) / I(0)\right)
$$

and the representation $R^{Y}$ in $\tilde{B}_{1}(Y)$ is a multiple of the regular representation $R$ of $Y$ in $A_{\rho^{\prime \prime}}(Y)$,

$$
R^{Y} \sim R \otimes m^{\prime}
$$

with multiplicity $m^{\prime}=\operatorname{dim} A_{\rho^{\prime}}(Z) / I(0)=m\left(\rho^{\prime}\right)$.

We claim that the operator $W$ takes the minimal ideal $I(Y)$ of $B_{1}$ into the minimal ideal $I(Y)$ of $B$. Indeed, if an element $\hat{f} \in B_{1}$ vanishes on a neighborhood of $Y$ and $\hat{\varphi} \in A_{\rho^{\prime}}(Z)$ is chosen as above, we get by (c)

$$
W(\hat{f})=W(\hat{\varphi} \hat{f})=\hat{\varphi} W(\hat{f})
$$

and the function $\hat{\varphi} W(\hat{f})$ vanishes on a neighborhood of $Y$. Thus $W$ defines a bounded dense range operator $\tilde{W}: B_{1} / I(Y) \rightarrow B / I(Y)$ which intertwines the representations $R^{Y}$ in these spaces. By Proposition 2(a),

$$
k\left(\lambda ; R^{Y} \mid \tilde{B}(Y)\right) \leq k\left(\lambda ; R^{Y} \mid \tilde{B}_{1}(Y)\right) \leq m\left(\rho^{\prime}\right) \leq m(\rho) .
$$

The lemma is proved.

3.3. Let now a LCA group $A=\mathbf{R}^{n} \oplus D$ be the sum of the vector group $\mathbf{R}^{n}$ and a finitely generated discrete group $D$. Then the dual group $G=\mathbf{R}^{n} \oplus X$ is a Lie group ( $X$ is a compact Lie group).

Given a weight $\rho$ on $A$ we can always replace it by a stronger one $\bar{\rho}=\rho^{\prime} \times \rho^{\prime \prime}$.

Indeed, the natural embedding $L_{\bar{\rho}}(A) \rightarrow L_{\rho}^{1}(A)$ defines a dense range bounded operator $W: A_{\bar{\rho}}(G) / I(\omega) \rightarrow \mathcal{A}_{\rho}(G) / I(\omega)$ which intertwines the representations $R^{\omega}$ in these spaces. By Proposition 2(a) the multiplicities $k\left(\pi ; R^{\omega}\right)$ can only increase after such a substitution.

So let us assume that $\rho=\rho^{\prime} \times \rho^{\prime \prime}$. Then the group algebra $L_{\rho}^{1}(A)$ factors into the tensor product

$$
L_{\rho}^{1}(A)=L_{\rho^{\prime}}^{1}\left(\mathbf{R}^{n}\right) \hat{\otimes} L_{\rho^{\prime \prime}}^{1}(D) .
$$

Respectively, $A_{\rho}(G)=A_{\rho^{\prime}}\left(\mathbf{R}^{n}\right) \hat{\otimes} A_{\rho^{\prime \prime}}(X)$. 
To proceed with the proof of Lemma 1 we introduce the algebra $A=C_{0}^{m}\left(\mathbf{R}^{n} ; B\right)$ $\left(B=A_{\rho^{\prime \prime}}(X)\right)$ of all $B$-valued $m$-smooth compactly supported functions $f: \mathbf{R}^{n} \rightarrow B$, with the usual sequence of seminorms,

$$
\|f\|_{m, Q}=\sum_{|\nu| \leq m}\left\|\left(\partial^{\nu} f\right)(x)\right\|_{L^{\infty}(Q)} .
$$

Here $\nu$ denotes a multi-index $\left(\nu_{1}, \ldots, \nu_{n}\right)$ with $|\nu|=\nu_{1}+\cdots+\nu_{n}, \partial^{\nu}$ a corresponding partial derivative and $Q$ runs over all compact subsets in $\mathbf{R}^{n}$. We consider the weight $\rho$ on $A$ which factors into the product $\rho^{\prime} \times \rho^{\prime \prime}$ with a polynomial constituent $\rho^{\prime}$ on $\mathbf{R}^{n}$.

As in the scalar case $(B=\mathbf{C})$, the Fourier algebra $A_{\rho}(G)=A_{\rho^{\prime}}\left(\mathbf{R}^{n}\right) \hat{\otimes} B$ is easily verified to contain $C_{0}^{m}\left(\mathbf{R}^{n}, B\right)$ as a dense subalgebra for $m=[n+1+\alpha]$, where $\alpha$ is the exponent of $\rho^{\prime}$.

We assume that a compact group $U$ acts by affine transformations on $\mathbf{R}^{n}$ and by translations with elements of a subgroup $Y=l(U)$ on $X$, where $l: U \rightarrow X$ is a group homomorphism. Of course this represents a special case of an affine action of $U$ on $G=\mathbf{R}^{n} \oplus X$.

Since $U$ acts by automorphisms of the Fourier algebra $A_{\rho}\left(\mathbf{R}^{n} \oplus X\right)$ and the dense subalgebra $A=C_{0}^{m}\left(\mathbf{R}^{n} ; B\right)$ is invariant under this action it suffices by Proposition 2 to prove finite multiplicity and estimate (3.1.1) for representations $R^{\omega}$ on $C_{0}^{m}\left(\mathbf{R}^{n} ; B\right){ }^{1}$

For this we have to study minimal ideals $I(\omega)$ of $A=C_{0}^{m}\left(\mathbf{R}^{n} ; B\right)$. They will be shown to have the following "Whitney-type" characterization.

Let $\omega$ be an orbit of $U$ in $\mathbf{R}^{n} \oplus X$, the Gelfand space of $\mathcal{A}$. We denote by $p$ and $q$ coordinate projections : $\mathbf{R}^{n} \oplus X \rightarrow \mathbf{R}^{n}$ and $q: \mathbf{R}^{n} \oplus X \rightarrow X$.

The image $\Omega=p(\omega)$ is an orbit of $U$ in $\mathbf{R}^{n}$, and for any point $a \in \Omega$, a subset $q\left(p^{-1}(a) \cap \omega\right)=\omega_{a}$ is an orbit of a stability subgroup $U_{a}$ in $X$. The orbit $\omega$ stratifies over its image $\Omega, \omega=\bigcup_{a \in \Omega}\left(a, \omega_{a}\right)$.

LEMMA 3. Let a compact group $U$ act by affine transformations on $\mathbf{R}^{n}$ and by translations with elements $\{y \in Y=l(U)\}$ (the image of $U$ under a group homomorphism $l: U \rightarrow X$ ) on $X$. For any orbit $\omega \subset \mathbf{R}^{n} \oplus X$, the minimal ideal $I(\omega)$ of the algebra $A=C_{0}^{m}\left(\mathbf{R}^{n} ; B\right)\left(B=A_{\rho^{\prime \prime}}(X)\right)$ has the characterization

$$
I(\omega)=\left\{f \in \mathcal{A}\left|\left(\partial^{\nu} f\right)(a) \in I\left(\omega_{a}\right) \forall a \in \Omega,\right| \nu \mid \leq m\right\} .
$$

Here $I\left(\omega_{a}\right)$ denotes the minimal ideal of $B$ with hull $\omega_{a} \subset X$.

The proof of Lemma 3 is long and requires a number of prerequisites We postpone it until §5. And now let us apply Lemma 3 to complete the proof of Lemma 1.

Lemma 3 can be reformulated in terms of so-called $m$-jets of smooth $B$-valued functions $f \in C_{0}^{m}\left(\mathbf{R}^{n} ; B\right)$. By an $m$-jet of $f \in C_{0}^{m}\left(\mathbf{R}^{n} ; B\right)$ we mean as usual a tuple of its partial derivatives

$$
J^{m}(f, x)=\left\{\left(\partial^{\nu} f\right)(x)\right\}_{|\nu| \leq m}
$$

considered as a continuous map from $\mathbf{R}^{n}$ into the product $B \otimes J^{m}$, where $J^{m}$ is a finite dimensional space of scalar $m$-jets, or equivalently, all polynomials in variables $x_{1}, \ldots, x_{n}$ of degree $\leq m$. Obviously, $\operatorname{dim} J^{m}=\left(\begin{array}{c}n+m \\ n\end{array}\right)$.

\footnotetext{
${ }^{1}$ Though $C_{0}^{m}\left(\mathbf{R}^{n} ; B\right)$ is not a Banach algebra on the whole space $\mathbf{R}^{n}$, one can always restrict it to a sufficiently large compact subset $Q \subset \mathbf{R}^{n}$, where $\|f\|_{m, Q}$ becomes a Banach norm.
} 
Formula (3.3.1) then becomes

$$
I(\omega)=\left\{f \in C_{0}^{m}\left(\mathbf{R}^{n} ; B\right) \mid J^{m}(f, x) \in I\left(\omega_{x}\right) \otimes J^{m} \forall x \in \Omega\right\} .
$$

The group $U$ acts by automorphisms on $\mathbf{R}^{n}$ and we denote this representation by $\tau, \tau(a)=a^{u}\left(a \in \mathbf{R}^{n}, u \in U\right)$. The representation $\tau$ extends to the space of all homogeneous polynomials (symmetric tensors) on $\mathbf{R}^{n}$ of degree $k(k=0,1, \ldots, m)$ and thus gives rise to a representation $\tau_{m}$ of $U$ on the space $J^{m}, d\left(\tau_{m}\right)=\left(\begin{array}{c}n+m \\ n\end{array}\right)$ (see Example 3 of the Introduction).

Notice that for a smooth function $f$,

$$
J^{m}\left(f\left(x^{\tilde{u}}\right), x\right)=\tau_{m}(u) J^{m}\left(f, x^{\tilde{u}}\right), \quad u \in U .
$$

Indeed, $x^{\tilde{u}}=x^{u}+l(u)$, the first term is linear in $x$, while the second vanishes after differentiation.

We apply Lemma 3 (in the form (3.3.2)) to embed the representation $R^{\omega}$ into some induced representation. To construct the induced representation let us consider the following $U$-homogeneous vector bundles over $\Omega$ :

(1) The Banach bundle $\left(\mathcal{E}^{\prime}, \Omega\right)$ with fibres $E^{\prime}(x)=B / I\left(\omega_{x}\right)(x \in \Omega)$ and a cocycle $R(x, u): \tilde{\varphi}(x) \rightarrow\left(R_{u} \varphi\right)^{\sim}\left(x^{\tilde{u}}\right)$.

Here $\tilde{\varphi}(x)$ denotes the image of an element $\varphi \in B$ in the quotient space $B / I\left(\omega_{x}\right)$, $R_{u}$ is the representation of $U$ on $B=A_{\rho^{\prime \prime}}(X)$ obtained from its action on $X$,

$$
\left(R_{u} \varphi\right)(y)=\varphi(y+l(u)) \quad(\varphi \in B, u \in U) .
$$

The topology on $\mathcal{E}^{\prime}$ is "induced" by the subspace of cross sections $\Gamma=\{\tilde{\varphi}(x) \mid$ $\left.(x \in \Omega) \forall \varphi \in C_{0}^{m}\left(\mathbf{R}^{n} ; B\right)\right\}$, which satisfies the following assumptions:

(I) The numerical functions $x \rightarrow\|\tilde{\varphi}(x)\|$ are continuous on $\Omega$ for all $\tilde{\varphi} \in \Gamma$.

(II) For each $x \in \Omega, \Gamma(x)=\{\tilde{\varphi}(x) \mid \forall \tilde{\varphi} \in \Gamma\}$ is equal to a fibre space $E^{\prime}(x)$.

Then $\left[5\right.$, Proposition 10.4] there exists a unique topology on $\mathcal{E}^{\prime}$ for making $\left(\mathcal{E}^{\prime}, \Omega\right)$ a Banach bundle such that all sections $\tilde{\varphi} \in \Gamma$ are continuous.

(2) The finite dimensional trivial bundle $\left(\mathcal{E}^{\prime \prime}, \Omega\right), \mathcal{E}^{\prime \prime}=J^{m} \times \Omega$, with the fibre space $J^{m}$ and a cocycle. $\tau_{m}(x, u)=\tau_{m}(u) \forall x \in \Omega$.

As usual one can form a tensor product bundle $(E, \Omega)=\left(\mathcal{E}^{\prime} \otimes \mathcal{E}^{\prime \prime}, \Omega\right)$, whose fibres are spaces $E(a)=\left(B / I\left(\omega_{a}\right)\right) \otimes J^{m}(a \in \Omega)$ and the cocycle $\tau(a, u)=R(a, u) \otimes \tau_{m}(u)$.

Now let us construct an intertwining operator $W$ from the space $A=C_{0}^{m}\left(\mathbf{R}^{n}, B\right)$ to the space of continuous sections $\Gamma(\Omega ; \mathcal{E})$ of the bundle $(\mathcal{E}, \Omega)$ as

$$
W: f \rightarrow F(x)=J^{m}(f, x) /\left(I\left(\omega_{x}\right) \otimes J^{m}\right) \in \Gamma(\Omega, \mathcal{E}) .
$$
of $A$.

By Lemma 3 (formula (3.3.2)) the kernel of $W$ is equal to the minimal ideal $I(\omega)$

Let us check that $W$ intertwines the representation $T$ of $U$ on $A$ and the induced representation $L=\operatorname{Ind}(U ; \tau, \mathcal{E})$ on $\Gamma(\Omega, \mathcal{E})$. The image of a function $f(x) \in$ $C_{0}^{m}\left(\mathbf{R}^{n} ; B\right)$ under the action of $T_{u}$ is equal to

$$
\left(T_{u} f\right)(x)=g(x)=R_{u}^{-1}\left[f\left(x^{\tilde{u}}\right)\right] .
$$

Computing the $m$-jet of $g(x)$ at the point $x \in \Omega$ we find

$$
\partial^{\nu}[g(x)]=R_{u}^{-1} \partial^{\nu}\left[f\left(x^{\tilde{u}}\right)\right]=R_{u}^{-1} \sum_{|\mu|=m} \tau_{\nu, \mu}(u)\left(\partial^{\mu} f\right)\left(x^{\tilde{u}}\right),
$$


$\left\{\tau_{\nu, \mu}\right\}_{|\nu|=|\nu|=m}$ being matrix entries of the representation $\tau_{m}$ in the "natural basis" of $J^{m}=\bigoplus_{k=0}^{m} S_{k}\left(\mathbf{R}^{n}\right)$. We can rewrite (3.3.4) in the vector form as

$$
J^{m}[g(x)]=\left(R_{u}^{-1} \otimes \tau_{m}(u)\right)\left[f\left(x^{\tilde{u}}\right)\right]
$$

which shows the intertwining relation.

Therefore $W$ defines an intertwining embedding of the representation $R^{\omega}$ (the quotient of $T$ modulo $I(\omega))$ into the induced representation $L=\operatorname{Ind}_{U_{x}}^{U}\left(R^{\omega} \otimes \tau_{m}\right)$.

On each fibre space $B / I\left(\omega_{a}\right) \otimes J^{m}$ we get a representation $R^{\omega_{a}} \otimes \tau_{m}$ of the stability subgroup $U_{a}$.

By Proposition 2 the multiplicities of $R^{\omega}$ are bounded by those of $L$,

$$
k\left(\sigma ; R^{\omega}\right) \leq k(\sigma ; L) \quad \forall \sigma \in \hat{U}
$$

and by the Frobenius reciprocity theorem (Proposition 1)

$$
k(\sigma ; L)=k\left(\sigma \mid U_{a} ; R^{\omega_{a}} \otimes \tau_{m}\right) .
$$

But the representation $R^{\omega_{a}}$ is of the type considered in Lemma 2 and hence FM while $\tau_{m}$ is finite dimensional. Then by Proposition $2, R^{\omega_{a}} \otimes \tau_{m}$ and therefore $L$ and $R^{\omega}$ ("embedded" in $L$ ) are also FM.

In order to estimate the multiplicities in the right-hand side of (3.3.6) we shall use

PROPOSITION 4. Let $R$ be an FM-representation of a compact group $V$ and $\sigma, \tau$ be finite dimensional representations. The intertwining number $k(\sigma ; R \otimes \tau)$ is estimated as

$$
k(\sigma ; R \otimes \tau) \leq \sup \left\{k(\mu ; R) \mid \mu \subset \sigma \otimes \tau^{*}\right\} d(\sigma) d(\tau),
$$

the supremum is taken over all irreducible $\mu \in \hat{V}$ which occur in the product $\sigma \otimes \tau^{*}$ ( $\sigma$ tensored with contragredient of $\tau$ ).

We decompose $R$ into the sum $\bigoplus_{\mu} k(\mu ; R) \otimes \mu$ of irreducible components $\mu \in \hat{V}$. Then the intertwining number $k(\sigma ; R \otimes \tau)$ is equal to

$$
k(\sigma ; R \otimes \tau)=\sum_{\mu} k(\mu, R) k(\sigma ; \mu \otimes \tau) \leq \sup \{k(\mu ; R)\} \sum_{\mu} k(\sigma ; \mu \otimes \tau) ;
$$

the summation is taken over all $\mu \in \hat{V}$ such that $k(\sigma ; \mu \otimes \tau)>0$. But $k(\sigma, \mu \otimes \tau)=$ $k\left(\mu ; \sigma \otimes \tau^{*}\right)$, since the spaces $\operatorname{Hom}_{V}(\sigma ; \mu \otimes \tau)$ and $\operatorname{Hom}_{V}\left(\mu ; \sigma \otimes \tau^{*}\right)^{*}$ are naturally isomorphic.

Since

$$
\sum_{\mu} k\left(\mu ; \sigma \otimes \tau^{*}\right) \leq \sum_{\mu} k\left(\mu ; \sigma \otimes \tau^{*}\right) d(\mu)=d(\sigma) d(\tau),
$$

we immediately get (3.3.7).

Let us apply Proposition 4 to the representations $R=R^{\omega_{a}}, \sigma=\sigma \mid U_{a}$ and $\tau=\tau_{m}$ of the group $V=U_{a}$.

We recall that a compact group $U$, consequently $U_{a}$, acts on the algebra $B=$ $A_{\rho^{\prime \prime}}(X)$ by translations with elements $\{y=l(v) \mid v \in U\}$. Let $Y_{0}=l\left(U_{a}\right)$. Each $U_{a}$-orbit is a coset $x+Y_{0}$ in $X$. Obviously, all $\mu$, which occur in $R^{\omega_{a}}$, are characters of $Y_{0}$ "pulled back" to $U_{a}$ and by Lemma 2 the multiplicities $k\left(\mu ; R^{\omega_{a}}\right) \leq m\left(\rho^{\prime \prime}\right)$. 
Therefore by (3.3.5), (3.3.6) and (3.3.7),

$$
\begin{aligned}
k\left(\sigma ; R^{\omega}\right) & \leq k\left(\sigma \mid U_{a} ; R^{\omega_{a}} \otimes \tau_{m}\right) \leq m\left(\rho^{\prime \prime}\right) d\left(\tau_{m}\right) d(\sigma) \\
& =c(n, \rho) d(\sigma) \quad \forall \sigma \in \hat{U} .
\end{aligned}
$$

This proves Lemma 1 in the case of a Lie group $G=\mathbf{R}^{n} \oplus X$ and a compact group $U$ acting by affine transformations on $\mathbf{R}^{n}$ and by translations on $X$.

3.4. Now we shall reduce the general affine action on $G=\mathbf{R}^{n} \oplus X$ to the above particular case.

But first let us study the automorphic and affine actions of a compact group $U$ on a compact abelian Lie group $X$.

PROPOSITION 5. Let a compact group $U$ act by affine transformations on a compact abelian Lie group $X$. There exists an open normal subgroup $V \subset U$, which acts on $X$ by translations, i.e.,

$$
v: x \rightarrow x+l(v) \quad(x \in X, v \in V),
$$

where $l: V \rightarrow X$ is a group homomorphism.

ProOF. First we note that the group of automorphisms of the compact abelian Lie group $X$ is discrete. Indeed, $X=\mathbf{T}^{k} \oplus F$, where $F$ is finite and the torus $\mathbf{T}^{k}=X_{0}$ is characteristic. So any automorphism of $X$ is uniquely determined by a triple $\left(u_{1}, h, u_{2}\right)$, where

$$
u_{1} \in \operatorname{Aut}^{k}=\operatorname{GL}(k, \mathbf{Z}), \quad u_{2} \in \operatorname{Aut} F, h \in \operatorname{Hom}\left(F ; \mathbf{T}^{k}\right) \cong F^{k} .
$$

Obviously, Aut $X=$ Aut $F \times\left(F^{k} \rtimes \mathrm{GL}(k, \mathbf{Z})\right)$ is countable and therefore discrete.

Let a compact group $U$ act on $X$ by automorphisms $x \rightarrow x^{u}$ and by affine transformations $x \rightarrow x^{\tilde{u}}=x^{u}+l(u)$. The kernel $V$ of a homomorphism $U \rightarrow \operatorname{Aut} X$ is a normal subgroup of finite index in $U$. Since the automorphic action of $V$ is trivial, the cocycle $l: U \rightarrow X$ restricted to $V$ turns into a homomorphism of $V$ into a compact abelian group $X$. We denote by $Y$ the image of $V$ in $X, Y$ is a closed subgroup of $X$. Obviously, the affine action of a subgroup $V \subset U$ on $X$ is given by translations by elements $\{y=l(v) \in Y\}$,

$$
x^{\tilde{v}}=x+l(v) \quad \forall x \in X, v \in V . \text { Q.E.D. }
$$

We shall make another simple observation.

PROPOSITION 6. Let $\omega \cong U_{1} \backslash U$ be a $U$-homogeneous space of a group $U$ and $V$ be a normal subgroup of finite index in $U$. Then $\omega$ splits into the union of disjoint $V$-orbits, $\omega=\bigcup_{s \in \omega^{\prime}} \omega_{s}$. Moreover, the set $\omega^{\prime}$ is finite and the group $U$ (actually the finite factor-group $U / V$ ) acts on $\omega^{\prime}$ transitively, i.e., $\omega^{\prime}$ is a finite U-homogeneous space.

The proof is obvious.

Now let $G=\mathbf{R}^{n} \oplus X$ be the sum of the vector group $\mathbf{R}^{n}$ and a compact Lie group $X$. Denote by $a \rightarrow a^{u}$ the automorphic action of $U$ on $G$ and by $a \rightarrow a^{\tilde{u}}=a^{u}+l(u)$ its affine action.

Later (Lemma 4, §4) we shall prove the existence of a $U$-invariant decomposition $G=\mathbf{R}^{n} \oplus X$ with respect to the automorphic action. Then the cocycle $l: U \rightarrow G$ splits into the sum of two cocycles $l_{1}: U \rightarrow \mathbf{R}^{n}$ and $l_{2}: U \rightarrow X$. 
Take an affine orbit $\omega$ of $U$ in $\mathbf{R}^{n} \oplus X$. By Proposition 5 there exists an open normal subgroup $V \subset U$ which acts on $X$ by translations with elements $\left\{l_{2}(v) \mid\right.$ $v \in V\}$. Then by Proposition $6, \omega=\bigcup_{s \in \omega^{\prime}} \omega_{s}$ is a finite union of disjoint $V$-orbits $\left\{\omega_{s}\right\}_{s \in \omega^{\prime}}$.

The quotient space $A / I(\omega)\left(\mathcal{A}=\mathcal{A}_{\rho}\left(\mathbf{R}^{n} \oplus X\right)\right)$ decomposes into the direct sum $\bigoplus_{s \in \omega^{\prime}} A / I\left(\omega_{s}\right)$, since $\left\{\omega_{s}\right\}_{s}$ are disjoint closed subsets of $G$.

The union $\mathcal{E}=\bigcup_{s \in \omega^{\prime}}\left(\mathcal{A} / I\left(\omega_{s}\right)\right)$ has a natural structure of a $U$-homogeneous vector bundle over the finite base $\omega^{\prime}$ and the representation $R^{\omega}$ is induced,

$$
R^{\omega}=\operatorname{Ind}_{U_{s}}^{U}\left(R^{\omega_{s}}\right)
$$

where $R^{\omega_{s}}$ is a representation of a stability subgroup $U_{s}$ on the quotient space $A / I\left(\omega_{s}\right)$.

Notice that the action of a subgroup $V$ on $\mathbf{R}^{n} \oplus X$ satisfies all the assumptions of Lemma 3. Namely, $V$ acts by affine transformations on $\mathbf{R}^{n}$ and by translations with elements $\{l(v) \mid v \in V\}$ on $X$. The representation $R^{\omega_{s}} \mid V$ on the quotient space $A / I\left(\omega_{s}\right)$ is obtained from this action, since $\omega_{s}$ is also a $V$-orbit.

Therefore, estimate (3.3.10) applies to $R^{\omega_{s}} \mid V$,

$$
k\left(\sigma ; R^{\omega_{s}} \mid V\right) \leq c(n, \rho) d(\sigma) \quad \forall \sigma \in \hat{V} .
$$

To get a similar estimate for the representation $R^{\omega}$ of the whole group $U$ we use

PROPOSITION 7. Let $S$ be a representation of a subgroup $U_{0} \subset U$. If there exists a subgroup $V \subset U_{0}$ such that the multiplicities

$$
k(\sigma ; S \mid V) \leq c d(\sigma) \quad \forall \sigma \in \hat{V},
$$

with some constant $c$, then a similar estimate holds for the induced representation $R=\operatorname{Ind}_{U_{0}}^{U}(S)$, i.e.,

$$
k(\pi ; R) \leq c d(\pi) \quad \forall \pi \in \hat{U}
$$

with the same constant $c$.

Indeed, by the Frobenius reciprocity theorem (Proposition 1) and the inclusion $V \subset U_{0}$ it follows that

$$
k(\pi ; R)=k\left(\pi \mid U_{0} ; S\right) \leq k(\pi|V ; S| V) .
$$

Decompose $\pi \mid V$ into the sum of isotypical components, $\pi \mid V=\bigoplus_{j} \sigma_{j} \otimes k_{j}\left(\sigma_{j} \in\right.$ $\hat{V})$ and substitute them into the right-hand side of (3.4.3). Then

$$
\begin{aligned}
k(\pi ; R) & \leq k(\pi|V ; S| V)=\sum_{j} k_{j} k\left(\sigma_{j} ; S \mid V\right) \\
& \leq c \sum_{j} k_{j} d\left(\sigma_{j}\right) \leq c d(\pi) . \quad \text { Q.E.D. }
\end{aligned}
$$

Proposition 7 applied to the representations $S=R^{\omega_{s}}$ and $R^{\omega}=\operatorname{Ind}_{U_{s}}^{U}\left(R^{\omega_{s}}\right)$ yields the estimate

$$
k\left(\pi ; R^{\omega}\right) \leq c(n, \rho) d(\pi) \quad \forall \pi \in \hat{U},
$$

and thus proves Lemma 1 for all Lie groups $G$ of the form $\mathbf{R}^{n} \oplus X$, with a compact Lie group $X$. 
3.5. We are now able to prove Lemma 1 for all compactly generated groups $A$, respectively Lie groups $G=\hat{A}$. As above a compact group $U$ acts on $G$ by automorphisms $a \rightarrow a^{u}$ and affine transformations $a \rightarrow a^{\tilde{u}}=a^{u}+l(u)$.

In the next section (Lemma 4) we shall establish the existence of subgroups $\mathbf{R}^{n}$ and compact $X$ of $G$ invariant under the automorphic action of $U$ and such that the factor group $D=G /\left(\mathbf{R}^{n} \oplus X\right)$ is discrete. In particular, $U$ acts by automorphisms on $D$.

Note that if a subgroup $B=\mathbf{R}^{n} \oplus X \subset G$ is stable under automorphisms of $U$, then the cocycle $l$ on $G$ projects on the factor group $D=G / B, i(u)=l(u) / B(u \in$ $U)$. So we also get an affine action of $U$ on $D$,

$$
u: d \rightarrow d^{\tilde{u}}=d^{u}+i(u) \quad(d \in D) .
$$

Two affine actions on $G$ and $D$ commute with the natural homomorphism $p: G \rightarrow$ $D=G / B$.

Let $\omega \subset G$ be an affine orbit of $U$. Its image $\omega^{\prime}=p(\omega)$ is a finite affine orbit of $U$ in $D$. As above we get a finite partition $\omega=\bigcup_{s \in \omega^{\prime}} \omega_{s}$ with $\omega_{s}=\omega \cap p^{-1}(s)$ an orbit of a stability subgroup $U_{s}$ in a coset $B_{s}=p^{-1}(s)=B+d$. Hence the representation $R^{\omega}$ is induced, $R=\operatorname{Ind}_{U_{s}}^{U}\left(R^{\omega_{s}}\right)$.

The translation with an element $d \in B_{s}$ takes a coset $B_{s}$ onto the subgroup $B$ and transforms the affine action of $U_{s}$ on $B_{s}$ into the new affine action on $B$ (cf. Example 5). Namely, $u: b \rightarrow b^{\tilde{u}}+\left(d^{u}-d\right)$.

Let us remark that the transformed action of $U_{s}$ on $B$ would be affine even if we started with an automorphic action on $G$.

But the group $B$ splits into the sum of $\mathbf{R}^{n}$ and a compact group $X$. So the previous result (3.4.4) applies and we get the estimate of multiplicities,

$$
k\left(\sigma, R^{\omega_{s}}\right) \leq c(n, \rho) d(\sigma) \quad \forall \sigma \in \hat{U}_{s} .
$$

By Proposition 7 the same estimate holds for the induced representation $R^{\omega}$,

$$
k\left(\pi ; R^{\omega}\right) \leq c(n, \rho) d(\pi) \quad \forall \pi \in \hat{U} .
$$

This completes the proof of Lemma 1.

4. Actions of compact groups on LCA groups (reduction lemma) and the proof of the main theorem.

4.1. After we have proved Theorem 1 for compactly generated groups $A$ the general case will be handled by a certain limit procedure. Here an important tool is the following "reduction lemma", which describes the structure of a LCA group $A$ upon which a compact group $U$ acts by automorphisms.

In a somewhat different setting a similar result was proved in $[8,1.1$. Theorem].

LEMMA 4. Let a compact group $U$ act by automorphisms on a LCA group A. Then: D.

(a) There exists a decomposition $A=\mathbf{R}^{n} \oplus D$ with $U$-stable subgroups $\mathbf{R}^{n}$ and

(b) The subgroup $D$ contains an open $U$-stable compact subgroup $K$. Therefore, $U$ acts by automorphisms on $\mathbf{R}^{n}, D, K$, and on the discrete factor group $\Gamma=D / K$.

(c) The group $\Gamma=D / K$ is equal to $\lim _{\alpha}$ ind $\Gamma_{\alpha}$ where $\Gamma_{\alpha}(\alpha \in \Sigma)$ runs over the family of all finitely generated $U$-stable subgroups of $\Gamma$. Consequently $D=$ 
$\lim _{\alpha}$ ind $D_{\alpha}$, where $D_{\alpha}=p^{-1}\left(\Gamma_{\alpha}\right)$ and $p$ denotes the natural homomorphisms $D \rightarrow$ $\Gamma=D / K$.

Proof. ${ }^{2}$ The identity component $A_{0}$ of $A$ is characteristic in $A$, hence $U$ invariant, and so is the maximal compact subgroup (unique!) $K_{0}$ of $A_{0}$. By structure theory [19], $A_{0}=K_{0} \oplus \mathbf{R}^{n}$, and so $\hat{A}_{0}=\hat{K}_{0} \oplus \mathbf{R}^{n}$, with $\mathbf{R}^{n}\left(=K_{0}^{\perp}\right)$ characteristic, hence $U$-invariant. We need to show that this splitting of $\hat{A}_{0}$ (and the dual splitting of $A_{0}$ ) can be made $U$-invariant.

This is really a problem in continuous cohomology. More generally, suppose $U$ acts on a LCA group $H$, leaving a closed subgroup $E$ invariant, and suppose $H=E \oplus F$ as topological groups. Then $U$ acts on $H / E=F$, but may not preserve the copy of $F$ inside $H$. However, one can define a cocycle $\varphi: U \times F \rightarrow E$ by

$$
b^{u}=b^{\alpha(u)}+\varphi(u, b) \text { for } u \in U, b \in F,
$$

where $b^{u}$ means the action of $u$ on $b$ viewed as an element of $H$, and where $\alpha(u)$ is the action of $u$ on $F$ identified with $H / E$. One can check that $\varphi \in Z^{1}(U, \operatorname{Hom}(F, E))$, and that $F$ can be adjusted to be $U$-invariant provided the cocycle $\varphi$ is a coboundary. Here Hom means "continuous Hom".

To come back to our situation, take $H=\hat{A}_{0}$ and $E=\mathbf{R}^{n}, F=\hat{K}_{0}$ (a discrete group). Then $\operatorname{Hom}(F, E)$ is a quasicomplete locally convex topological vector space, so as is well known, $H^{k}(U, \operatorname{Hom}(F, E))=0$ for $k>0$. (See, for instance, A. Guichardet, Cohomologie des groupes topologiques et des algèbres de Lie, CEDIC/Nathan, Paris, 1980, p. 186.) In particular, $K_{0}^{\perp}$ has a $U$-invariant complement $\left(\cong \mathbf{R}^{n}\right)$ in $A_{0}$.

The same argument can be repeated to show that this $U$-invariant copy of $\mathbf{R}^{n}$ must have a $U$-invariant complement $D=A / \mathbf{R}^{n}$ in $A$, with of course $D \supset K_{0}$. Here we use the fact from the structure theory that $A=\mathbf{R}^{n} \oplus\left(A / \mathbf{R}^{n}\right)$ as LCA groups. This proves (a).

Now note that $D / K_{0}$ is totally disconnected, hence has a neighbourhood base for the identity $\left\{N_{\alpha}\right\}$ consisting of compact-open subgroups. If we fix $\alpha$ then for $N_{\beta}$ sufficiently small, the image of $N_{\beta}$ under the action of $U$ must still be contained in $N_{\alpha}$. Hence $U\left(N_{\beta}\right)$ generates a compact-open $U$-invariant subgroup of $D / K_{0}$, and thus $D / K_{0}$ has arbitrarily small $U$-invariant compact-open subgroups.

In particular, we can fix a $U$-stable compact-open subgroup $K$ of $D$. Let $\Gamma=$ $D / K$, which is discrete. $\Gamma$ is the inductive limit of its finitely generated subgroups, and since the $U$-orbit of any element of $\Gamma$ is finite we can take these $U$-stable without losing finite generation. This proves (b) and (c).

Lemma 4 applied to the dual group $G$ of $A$ and the dual action of $U$ on $G$ yields

COROLlaRY 1. (a) $G$ admits a $U$-invariant decomposition $G=\mathbf{R}^{n} \oplus \hat{D}$.

(b) $\hat{D}$ contains an open compact $U$-invariant subgroup $X=K^{\perp} \cong \hat{\Gamma}$.

(c) If $Y_{\alpha}=D_{\alpha}^{\perp}(\alpha \in \Sigma)$, then $\left\{Y_{\alpha}\right\}_{\alpha}$ are small $U$-stable subgroups of $X$, i.e., factor-groups $X_{\alpha}=X / Y_{\alpha}$ and $\hat{D}_{\alpha}=\hat{D} / Y_{\alpha}$ are Lie groups (not necessarily connected). Moreover

$$
X=\lim _{\alpha} \operatorname{pr} X_{\alpha}, \quad \hat{D}=\lim _{\alpha} \operatorname{pr} \hat{D}_{\alpha}
$$

${ }^{2}$ This short proof of Lemma 4 was proposed by the referee. 
and

$$
G=\lim _{\alpha} \operatorname{pr} G_{\alpha}, \quad \text { where } G_{\alpha}=\mathbf{R}^{n} \oplus \hat{D}_{\alpha} .
$$

4.2. Now let us study the structure of orbits $\omega \subset G$ under the affine action of $U$ on $G$.

We fix a $U$-stable decomposition of Lemma 4 and Corollary $1, G=\mathbf{R}^{n} \oplus \hat{D}$ with $\hat{D} \supset X=K^{\perp}$ and $X \supset Y_{\alpha}$. Denote by $\left\{p_{\alpha}\right\}_{\alpha \in \Sigma}$ and $p$ the natural homomorphisms

$$
\begin{gathered}
p_{\alpha}: G=\mathbf{R}^{n} \oplus \hat{D} \rightarrow G_{\alpha} \cong \mathbf{R}^{n} \oplus \hat{D} / Y_{\alpha}=\mathbf{R}^{n} \oplus \hat{D}_{\alpha}, \\
p: G \rightarrow \hat{K} \cong G /\left(\mathbf{R}^{n} \oplus X\right) .
\end{gathered}
$$

Notice that all these homomorphisms commute with the automorphic action of $U$ on an appropriate group $\left(G, \hat{K}, \mathbf{R}^{n}, X, \hat{D}\right.$, etc. $)$.

Furthermore since all the subgroups $\left(\mathbf{R}^{n}, X, Y_{\alpha}\right.$, etc.) of $G$ are invariant under the automorphic action the cocycle $l(u)$ projects on factor groups $\left(\hat{K}, G_{\alpha}\right.$, etc.) and thus gives rise to affine actions of $U$ on those, which commute with natural homomorphisms $p$ and $\left\{p_{\alpha}\right\}$.

We denote by $B$ the subgroup $\mathbf{R}^{n} \oplus X \subset G$ and for $s \in \hat{K}$ we denote by $B_{s}$ the coset $p^{-1}(s)=B+d\left(d \in p^{-1}(s)\right)$.

Let $\omega \subset G$ be an affine orbit of $U$. The image $\omega^{\prime}=p(\omega)$ is an orbit of $U$ in the discrete group $\hat{K}$, so $\omega^{\prime}$ is finite. Fix a point $s \in \omega^{\prime}$ and let $\omega_{s}=\omega \cap B_{s}$. Obviously $\omega_{s}$ is an orbit of a stability subgroup $U_{s}$ in the coset $B_{s}$.

Similarly the set $\omega_{s, \alpha}=p_{\alpha}\left(\omega_{s}\right)$ is an orbit of $U_{s}$ in a coset $B_{s, \alpha}=p_{\alpha}\left(B_{s}\right)=$ $d_{\alpha}+B_{\alpha}$, where $B_{\alpha}=\mathbf{R}^{n} \oplus \hat{D}_{\alpha}, d_{\alpha}=p_{\alpha}(d)$.

It is easy to see that the compact set $\omega_{s}$ is a projective limit of its images $\omega_{s, \alpha} \subset B_{s, \alpha}$,

$$
\omega_{s}=\lim _{\alpha} \operatorname{pr} \omega_{s, \alpha}
$$

4.3. Now we are able to complete the proof of Theorem 1. Since $\omega=\bigcup_{s \in \omega^{\prime}} \omega_{s}$ is a disjoint union of closed subsets we get as in the proof of Lemma 1 (§3.4) a decomposition

$$
A / I(\omega)=\bigoplus_{s \in \omega^{\prime}} A / I\left(\omega_{s}\right), \quad A=A_{\rho}\left(\mathbf{R}^{n} \oplus \hat{D}\right) .
$$

The union $\mathcal{E}=\bigcup_{s \in \omega^{\prime}} A / I\left(\omega_{s}\right)$ has a structure of a $U$-homogeneous vector bundle over the finite base $\omega^{\prime}$ and the representation $R^{\omega}$ is induced by the representation $R^{\omega_{s}}$ of $U_{s}$ on the quotient space $A / I\left(\omega_{s}\right)$,

$$
R^{\omega}=\operatorname{Ind}_{U_{s}}^{U}\left(R^{\omega_{s}}\right) .
$$

We shall realize $R^{\omega_{s}}$ as a Banach inductive limit of the representations $R^{\omega_{s, \alpha}}$ on factor groups $G_{\alpha}$. Denote by $B, B_{\alpha}(\alpha \in \Sigma)$ the Fourier algebras $A_{\rho}\left(\mathbf{R}^{n} \oplus \hat{D}\right)$ and $A_{\rho}\left(\mathbf{R}^{n} \oplus \hat{D}_{\alpha}\right)$, the second considered as a subalgebra of $B$ under the natural "pull-back" embedding

$$
p_{\alpha}^{*}: \hat{\phi} \rightarrow \hat{\phi} \circ p_{\alpha} \quad\left(\hat{\phi} \in B_{\alpha}\right)
$$

By (4.2.1) the monomorphism $p_{\alpha}^{*}$ takes the minimal ideal $I\left(\omega_{s, \alpha}\right)$ of $B_{\alpha}$ into the minimal ideal $I\left(\omega_{s}\right)$ of $B$ (this is true for any closed subset $Q=\lim \operatorname{pr}_{\alpha} Q_{\alpha}, Q_{\alpha} \subset$ $\left.G_{\alpha}\right)$. So $p_{\alpha}^{*}$ defines a bounded operator

$$
W_{\alpha}: B_{\alpha} / I\left(\omega_{s, \alpha}\right) \rightarrow B / I\left(\omega_{s}\right)
$$


The operator $W_{\alpha}$ intertwines the representations $R^{\omega_{s, \alpha}}$ and $R^{\omega_{s}}$ of the group $U_{s}$ in these spaces.

Moreover, the linear span of images $\left\{\operatorname{Im} W_{\alpha}\right\}_{\alpha}$ is obviously dense in $B / I\left(\omega_{s}\right)$. So $R^{\omega_{s}}$. So $R^{\omega_{s}}$ is a Banach inductive limit of representations $\left\{R^{\omega_{s, \alpha}}\right\}_{\alpha}$ in the sense of Proposition 3 ( $\S 1.2)$.

Each representation $R^{\omega_{s, \alpha}}$ is obtained from the action of $U$ on the Lie group $G_{\alpha}=\mathbf{R}^{n} \oplus \hat{D}_{\alpha}$. By Lemma 1 the representation $R^{\omega_{s, \alpha}}$ is FM and its multiplicities are estimated as

$$
k\left(\pi ; R^{\omega_{s, \alpha}}\right) \leq c(n, \rho) d(\pi) \quad \forall \pi \in \hat{U}_{s}
$$

with the constant $c(n, \rho)$ depending only on $n=\operatorname{dim} \mathbf{R}^{n}$ and $\rho$. Then Proposition 3 implies the same estimate for the Banach inductive limit $R^{\omega_{s}}=\lim _{\alpha}$ ind $R^{\omega_{s, \alpha}}$, and by Proposition 7 it remains true for the induced representation $R^{\omega}=\operatorname{Ind}_{U_{s}}^{U}\left(R^{\omega_{s}}\right)$.

The theorem is proved.

REMARK 1. The above argument suggests that Theorem 1 should be true for more general actions of $U$ on $G$, namely the action by automorphisms of the Fourier algebra $A_{\rho}(X)$. Obviously this group contains all affine transformations $\operatorname{Aff}(G)$ but may be bigger than the latter.

While groups of automorphisms of weighted Fourier algebras are unknown in general they were systematically studied in the case of the trivial weight $\rho \equiv 1$, i.e., the Fourier algebra $A(G)$ (see, for instance [22]). Any automorphism $u$ of $A(G)$ is given by a so-called piecewise-affine transformation $u: G \rightarrow G$. By definition, for any such $u$ there exists a partition $G=\bigcup_{\nu} B_{\nu}$ into the union of open disjoint cosets $B_{\nu}$ such that the map $u: B_{\nu} \rightarrow B_{u(\nu)}$ is affine, i.e.,

$$
u(a+b-c)=u(a)+u(b)-u(c) \quad \forall a, b, c \in B_{\nu} .
$$

Of course, an affine action of a group $V$ on a coset $B_{\nu}=B+d$ in $G$ defines (via translation) another affine action on an open subgroup $B$ of $G$ (see Example 5).

If we had a joint "open-coset" partition of $G$ for all elements $u \in U$ we could easily (via Propositions 5,6) reduce the action of $U$ by automorphisms of $A(G)$ to the case of an affine action of $V=U_{x}$ on an open subgroup $B \subset G$.

We do not know if for any compact group of piecewise affine transformations on $G$ there exists a joint "open-coset" partition.

This is obviously true for any finite family of transformations $\left(u_{1}, \ldots, u_{n}\right)$ and therefore for any topologically finitely generated group $U$ (i.e., a group which contains a finitely generated dense subgroup), in particular for any compact Lie group $U$.

We can formulate partial generalization of Theorem 1 along these lines.

THEOREM 2. If a topologically-finitely generated compact group $U$ acts by automorphisms of the Fourier algebra $A(G)$ on a $L C A$ group $G$, then for any orbit $\omega \subset G$, the representation $R^{\omega}$ is $F M$ and the multiplicities $k\left(\pi ; R^{\omega}\right)$ satisfy

$$
k\left(\pi ; R^{\omega}\right) \leq\left(\begin{array}{c}
2 n+1 \\
n+1
\end{array}\right) d(\pi) \quad \forall \pi \in \hat{U},
$$

$n$ being the dimension of the vector component of $X$.

Indeed, the weights $\rho^{\prime}$ and $\rho^{\prime \prime}$ are trivial here. Hence $m\left(\rho^{\prime \prime}\right)=1, \alpha\left(\rho^{\prime}\right)=0, m=$ $n+1$ and the constant $c(n, \rho)=\left(\begin{array}{c}2 n+1 \\ n+1\end{array}\right)$. 


\section{A generalized version of Whitney's theorem and the proof of} Lemma 3.

5.1. Let $B$ be a Banach space. We consider the space $A=C_{0}^{m}\left(\mathbf{R}^{n} ; B\right)$ of all $m$-smooth compactly supported functions $f: \mathbf{R}^{n} \rightarrow B$, equipped with the usual sequence of Sobolev's $m$-seminorms,

$$
\|f\|_{m, Q}=\sum_{|\nu| \leq m}\left\|\partial^{\nu} f\right\|_{L^{\infty}(Q)},
$$

$Q$ running over all compact subsets in $\mathbf{R}^{n}$. Given a function $f \in C^{m}\left(\mathbf{R}^{n}, B\right)$ we define its $m$-jet at a point $x \in \mathbf{R}^{n}$ as a tuple of partial derivatives

$$
J^{m}(f, x)=\left\{\left(\partial^{\nu} f\right)(x)\right\}_{|\nu| \leq m} \in J^{m} \otimes B .
$$

Here $J^{m}=J^{m}\left(\mathbf{R}^{n}\right)$ denotes the finite dimensional space of all scalar $m$-jets, equivalently all polynomials in variables $\left(x_{1}, \ldots, x_{n}\right)$ of degree $\leq m$.

Multiplication with scalar functions $\phi \in C_{0}^{m}\left(\mathbf{R}^{n}\right)$ defines a $C_{0}^{m}$-module structure on the space $A=C_{0}^{m}\left(\mathbf{R}^{n} ; B\right)$.

Let $\mathcal{M}$ be a submodule of $A$. For any point $x \in \mathbf{R}^{n}$ we denote by $\mathcal{M}(x)$ the localization of $\mathcal{M}$ at $x$,

$$
\mathcal{M}(x)=\left\{J^{m}(f, x) \mid \forall f \in \mathcal{M}\right\} \subset J^{m} \otimes B,
$$

and by $\bar{M}(x)$ the closure of $\mathcal{M}(x)$ in $J^{m} \otimes B$. We set

$$
\tilde{\mathcal{M}}=\left\{f \in \mathcal{A} \mid J^{m}(f, x) \in \overline{\mathcal{M}}(x) \forall x \in \mathbf{R}^{n}\right\} .
$$

A generalized version of Whitney's theorem is formulated as follows.

CONJECTURE. The closure $\overline{\mathcal{M}}$ of any submodule $\mathcal{M} \subset A$ is equal to $\tilde{\mathcal{M}}$. In particular any closed submodule $\mathcal{M} \subset \mathcal{A}$ admits the characterization

$$
\mathcal{M}=\left\{f \in \mathcal{A} \mid J^{m}(f, x) \in \overline{\mathcal{M}}(x) \forall x \in \mathbf{R}^{n}\right\} .
$$

This result was first proved in $[\mathbf{2 6}]$ for scalar functions $(\operatorname{dim} B=1)$ and later for vector-valued functions with values in a finite dimensional vector space $B$ (see, for instance [16, Chapter 2]).

Let us notice that the finite dimensional argument [16, Chapter 2] carries over verbatim to prove a weaker version of (5.1.4), namely: if $\mathcal{M}$ is closed, then

$$
\mathcal{M}=\left\{f \in \mathcal{A} \mid J^{m}(f, x) \in \mathcal{M}(x) \forall x \in \mathbf{R}^{n}\right\}
$$

(here localizations $\mathcal{M}(x)$ are substituted for their closures $\bar{M}(x)$ ).

But simple examples show that localizations $\{\mathcal{M}(x)\}_{x \in \mathbf{R}^{n}}$ may not be closed (even for closed $\mathcal{M}$ ) in the infinite dimensional case ${ }^{3}$ (they trivially are, if $\operatorname{dim} B<$ $\infty)$. This principal difference between two cases makes the first (strong) version of Whitney's theorem for Banach vector-valued functions much more difficult. We cannot prove it in general.

What we are able to prove here is a rather special case, yet sufficient for our purpose (Lemma 3 of $\S 3$ ). Namely, we consider a commutative regular and Wiener Banach algebra $B$ with the Gelfand space $X$. Then $A=C_{0}^{m}\left(\mathbf{R}^{n}, B\right)$ is also a commutative algebra with the Gelfand space $\mathbf{R}^{n} \times X$. We assume that a compact group $U$ acts by affine transformations on $\mathbf{R}^{n}$ and by automorphisms on $B$.

\footnotetext{
${ }^{3}$ This will typically happen in our setting, as explained below.
} 
The class of modules to be considered are the minimal ideals $\mathcal{M}=I(\omega)$ of the algebra $A$ corresponding to orbits $\omega \subset \mathbf{R}^{n} \times X$. Under certain assumptions on $B$ and the action of $U$ on $B$ (to be specified later on) we shall prove the equality $I(\omega)=$ $(I(\omega))^{\sim}$ for these modules. This result formulated as Lemma 3 was important in the proof of Theorem 1.

Lemma 3 will follow from the results of this section. The first three of them (Theorem 3, Lemmas 5 and 6) reduce the problem from the space of functions $C^{m}\left(\mathbf{R}^{n} ; B\right)$ to certain spaces of smooth cross-sections of some $U$-homogeneous vector bundles over the orbit $\Omega \subset \mathbf{R}^{n}$. Roughly speaking we show that for any function $f \in C^{m}\left(\mathbf{R}^{n} ; B\right)$ its $m$-jet $J^{m}(f, x)$ restricted on $\Omega$ is uniquely determined by the set of independent "normal derivatives" of $f$. Then in (5.5) we reformulate the problem for vector bundles on which a Lie group $U$ acts smoothly. Here invariant differential operators (infinitesimal operators of induced representations) play the role of partial derivatives $\partial^{\nu}$. Under certain assumptions (see $\S 5.6$ ) we prove the equality of modules $I(\omega)$ and $I(\omega))^{\sim}$ realized in spaces of smooth sections (Theorem 4). Finally (5.7) we verify the assumptions of Theorem 4 in the setting of Lemma 3 , i.e., when $B=A_{\rho}(X)$ is the Fourier algebra on a compact abelian Lie group $X$ and $U$ acts on $X$ by translations.

Let us remark that our argument is quite different from the one known in the finite dimensional case (cf. [26 and 16, Chapter 2]). It is heavily based upon some peculiar features of our setting, particularly the following two:

(a) A homogeneity of spaces, bundles, modules, etc. with respect to the action of a compact Lie group $U$.

(b) A possibility to study explicitly local (nonclosed) modules $\{\mathcal{M}(x) \mid x \in \Omega\}$ for $\mathcal{M}=I(\omega)$ and $\mathcal{M}=(I(\omega))^{\sim}$.

This study (§5.6) also reveals why localizations $\mathcal{M}(x)$ are not closed for $\mathcal{M}=I(\omega)$ in general, the reason being an "interplay" between two actions of $U$. The action on $\mathbf{R}^{n}$ results in an induced representation $L$ on spaces of smooth cross-sections over $\Omega$, while the action on $X$ gives rise to a representation $R$ on $B$. In a somewhat inaccurate form we can say that the interplay between $R$ and $L$ causes "local values" $\left(\partial^{\nu} f\right)(a) \in B$ to be "smooth" with respect to $R$, i.e. $\left(\partial^{\nu} f\right)(a)$ an element of a dense subspace $I\left(\omega_{a}\right) \cap B_{m}$, whose $B$-closure is $I\left(\omega_{a}\right)$. Here $B_{m}$ denotes the space of $m$-smooth vectors of the representation $R$ (see $\S 5.3$ ).

5.2. We shall start with a construction of some smooth vector bundles over orbits $\Omega$ of the group $U$ in $\mathbf{R}^{n}$ or more generally over smooth manifolds $\Omega \subset \mathbf{R}^{n}$ and discuss spaces of smooth cross-sections on these bundles. These bundles will be used to decompose the trivial jet-bundle over $\Omega$ into "tangential" and "normal" components. We shall also show that "purely" normal components uniquely determine the jet $\left(\partial^{\nu} f\right)_{|\nu| \leq m}$ restricted on $\Omega$.

The purpose of this study is to enable us to reformulate the problem (Whitney's theorem) in terms of smooth sections of normal bundles.

Let $\Omega \subset \mathbf{R}^{n}$ be a smooth closed submanifold. We consider $\mathbf{R}^{n}$ equipped with the natural inner product

$$
a \cdot x=\sum_{1}^{n} a_{i} x_{i} \quad\left(a, x \in \mathbf{R}^{n}\right) .
$$


Since tangent spaces $T_{a}=T_{a}(\Omega)(a \in \Omega)$ are embedded in $\mathbf{R}^{n}$, the inner product (5.2.1) induces a Riemannian metric on $\Omega$.

We consider the following smooth vector bundles over $\Omega$ :

(1) The trivial bundle $\Omega \times \mathbf{R}^{n}$ equipped with the metric (inner product) (5.2.1).

(2) The tangent bundle $(\tau, \Omega)$ with fibres $T_{a}$ (the tangent space of $\Omega$ at $a \in \Omega$ ), considered as a subbundle of $\Omega \times \mathbf{R}^{n}$

(3) The normal bundle $(\mathcal{N}, \Omega)$ whose fibres $N_{a}$ are orthogonal complements of $T_{a}$ in $\mathbf{R}^{n}$ with respect to the inner product (5.2.1).

Obviously, $\Omega \times \mathbf{R}^{n}$ is a direct orthogonal sum

$$
\Omega \times \mathbf{R}^{n}=(\tau \oplus \mathcal{N}, \Omega) .
$$

We shall use the same notations $\tau, \mathcal{N}$ also for complexifications $\left(\tau \otimes_{\mathbf{R}} \mathbf{C}, \Omega\right)$ and $\left(\mathcal{N} \otimes_{\mathbf{R}} \mathbf{C}, \Omega\right)$ considered as subbundles of $\Omega \times \mathbf{C}^{n}$.

Let $S_{k}\left(\mathbf{R}^{n}\right)$ denote the space of all symmetric $k$-tensors over $\mathbf{R}^{n}$ and $S_{k}(\tau)$, $S_{k}(\mathcal{N})$ the $k$ th symmetric tensor power of the bundles $\tau$ and $\mathcal{N}$. Obviously, the trivial bundle $\Omega \times S_{m}\left(\mathbf{R}^{n}\right)$ decomposes into the direct sum

$$
\Omega \times S_{m}\left(\mathbf{R}^{n}\right)=\bigoplus_{k+l=m} S_{k}(\tau) \otimes S_{l}(\mathcal{N}) .
$$

The sum in (5.2.3) is orthogonal with respect to the metric ("unitary structure") induced on each fibre space $S_{k}\left(\mathbf{R}^{n}\right)$ by the inner product (5.2.1) on $\mathbf{R}^{n}$. The trivial jet-bundle $\Omega \times J^{m}\left(\mathbf{R}^{n}\right)$ is the sum $\bigoplus_{r=0}^{m} \Omega \otimes S_{r}\left(\mathbf{R}^{n}\right)$ and hence decomposes as $\bigoplus_{k+l<m} S_{k}(\tau) \otimes S_{l}(\mathcal{N})$.

We denote by $\mathcal{E}^{(r)}$ and $\mathcal{E}_{k, l}$ the bundles $\Omega \times S_{r}\left(\mathbf{R}^{n}\right)(r=0,1, \ldots, m)$ and $S_{k}(\tau) \otimes$ $S_{l}(\mathcal{N})(k, l=0,1, \ldots)$, respectively. Among those we are especially interested in normal bundles $S_{l}(\mathcal{N})$.

Given a Banach space $B$ let us define the following Banach vector bundles, each of which is a tensor product of a finite dimensional bundle $(\mathcal{E}, \Omega)$ and the trivial Banach bundle $\Omega \times B$ :

(4) $\Omega \times\left(\mathbf{C}^{n} \otimes B\right)$.

(5) Its subbundles $(\tau \otimes B, \Omega)$ and $(\mathcal{N} \otimes B, \Omega)$.

(6) "Symmetric-tensor" bundles $\left(\mathcal{E}_{k, l} \otimes B, \Omega\right)(k, l \geq 0)$ and $\left(\mathcal{E}^{(r)} \otimes B, \Omega\right)(r \geq 0)$.

If a finite dimensional bundle $(\mathcal{E}, \Omega)$ possesses a metric (unitary structure), then the product $(\mathcal{E} \otimes B, \Omega)$ is identified with the bundle $(\operatorname{Hom}(\mathcal{E}, B) ; \Omega)$, whose fibres are the spaces $H_{a}=\operatorname{Hom}\left(E_{a} ; B\right)(a \in \Omega)$. There is a natural (operator) norm in each $H_{a}$, which makes $\operatorname{Hom}(\mathcal{E} ; B)$ a Banach bundle in the sense of [5], and a smooth vector bundle in the usual ("finite dimensional") sense (cf. [25, Chapter 1]).

In this way one can identify bundles $\left(\mathcal{E}^{(r)} \otimes B, \Omega\right),\left(\mathcal{E}_{k, l} \otimes B, \Omega\right)$, etc. with bundles of symmetric multilinear forms $\left(P^{(r)}(B) ; \Omega\right)$ and $\left(P_{k, l}(B) ; \Omega\right)$ whose fibres are the spaces $P^{(r)}(a)$ and $P_{k, l}(a)$. Here a form $\phi \in P^{(r)}(a)$,

$$
\phi: \mathbf{R}^{n} \underbrace{\times \cdots \times \mathbf{R}^{n} \rightarrow B,}_{r \text { times }}
$$

is symmetric in all variables, while $\phi \in P_{k, l}(a)$,

$$
\phi: T_{a} \underbrace{\times \cdots \times}_{k \text { times }} T_{a} \times N_{a} \underbrace{\times \cdots \times}_{l \text { times }} N_{a} \rightarrow B,
$$


has a mixed $(k, l)$ symmetry (in $k$-tangent and $l$-normal variables separately).

The norm of an $r$-form $\phi: \mathbf{R}^{n} \times \cdots \times \mathbf{R}^{n} \rightarrow B$ is given by

$$
\|\phi\|=\sup _{\left\{\left\|\xi_{i}\right\| \leq 1\right\}}\left\|\phi\left(\xi_{1}, \ldots, \xi_{r}\right)\right\| \text {. }
$$

If we fix a basis $\left(e_{1}, \ldots, e_{n}\right)$ in $\mathbf{R}^{n}$ and denote by $f_{\nu} \in B$ the $\nu$ th "matrix entry" of a multilinear form $\phi, \nu=\left(\nu_{1}, \ldots, \nu_{n}\right)$, i.e.

$$
f_{\nu}=\phi(\underbrace{e_{1}, \ldots, e_{1}}_{\nu_{1} \text { times }} ; \cdots \underbrace{e_{n}, \ldots, e_{n}}_{\nu_{n} \text { times }}),
$$

then (5.2.6) is equivalent to the norm

$$
\|\phi\|=\sum_{|\nu|=r}\left\|f_{\nu}\right\|
$$

Since all the above Banach vector bundles are smooth in the obvious sense we can introduce the following spaces of smooth sections: $\Gamma^{m}\left(\Omega \times \mathbf{C}^{n}\right)=C^{m}\left(\Omega ; \mathbf{R}^{n}\right)$; $\Gamma^{m}(\Omega ; \tau) ; \Gamma^{m}\left(\Omega ; \mathcal{E}^{(r)} \otimes B\right) ; \Gamma^{m}\left(\Omega ; \mathcal{E}_{k, l} \otimes B\right)$, etc. $(m=0,1, \ldots, \infty)$.

These spaces have a natural structure of modules over the algebra of $m$-smooth functions $C^{m}(\Omega)$. As in (5.2.4), (5.2.5) they can also be characterized in terms of multilinear "module-maps". For instance, $\Gamma^{m}\left(\Omega ; \mathcal{E}^{(r)} \otimes B\right)$ is identified with the space of all $m$-smooth symmetric multilinear forms from the $C^{m}(\Omega)$-module $\Gamma^{m}\left(\Omega ; \mathbf{R}^{n}\right)$ into the $C^{m}(\Omega)$-module $C^{m}(\Omega ; B)$,

$$
\phi: \Gamma^{m}\left(\Omega ; \mathbf{R}^{n}\right) \underbrace{\times \cdots \times}_{r \text { times }} \Gamma^{m}\left(\Omega ; \mathbf{R}^{n}\right) \rightarrow C^{m}(\Omega ; B),
$$

s.t.

$$
\phi\left(f \xi_{1} ; \ldots ; \xi_{r}\right)=f \phi\left(\xi_{1}, \ldots, \xi_{r}\right) \quad \forall f \in C^{m}(\Omega), \xi_{1} \cdots \xi_{r} \in \Gamma^{m}\left(\Omega, \mathbf{R}^{n}\right)
$$

and

$$
\phi\left(\ldots \xi_{i}, \ldots, \xi_{j} \ldots\right)=\phi\left(\ldots \xi_{j} \ldots \xi_{i} \ldots\right) \quad \forall i<j .
$$

Similarly elements $\phi \in \Gamma^{m}\left(\Omega ; \mathcal{E}_{k, l} \otimes B\right)$ map

$$
\Gamma^{m}(\Omega ; \tau) \underbrace{\times \cdots \times}_{k \text { times }} \Gamma^{m}(\Omega ; \tau) \times \Gamma^{m}(\Omega ; \mathcal{N}) \underbrace{\times \cdots \times}_{l \text { times }} \Gamma^{m}(\Omega ; \mathcal{N}) \rightarrow C^{m}(\Omega, B)
$$

and have a $(k, l)$-mixed symmetry

As in (5.2.3) we get a decomposition

$$
\Gamma^{m}\left(\Omega ; \mathcal{E}^{(r)} \otimes B\right)=\bigoplus_{k+l=r} \Gamma^{m}\left(\Omega ; \mathcal{E}_{k, l} \otimes B\right)
$$

A $(k, l)$-component of a section $\phi \in \Gamma^{m}\left(\Omega ; \mathcal{E}^{(r)} \otimes B\right)$ is obtained by restriction of the form $\phi$ onto the subspace of all tuples $\left(\xi_{1}, \ldots, \xi_{k}, \eta_{1}, \ldots, \eta_{l}\right)$ of $k$ tangent fields $\xi_{i} \in \Gamma^{m}(\Omega ; \tau)$ and $l$ normal fields $\eta_{j} \in \Gamma^{m}(\Omega ; \mathcal{N})$.

5.3. Next we want to introduce "partial derivatives" and Sobolev's $m$-norms in spaces of smooth sections. There are different ways of doing this.

The standard definition uses "local coordinates" on the bundle $(\Omega ; \mathcal{E})$ and a partition of unity on $\Omega$. Precisely, a local trivialization on $(\Omega, \mathcal{E})$ defines a "pullback" isomorphism between a subspace $\Gamma_{0}^{m}(\Delta ; \mathcal{E})$ of all sections supported in a 
local neighborhood $\Delta \subset \Omega$ and the space $C_{0}^{m}\left(\mathbf{R}^{k} ; E\right), k=\operatorname{dim} \Omega ; E \cong E_{x}(x \in \Omega)$. Then the $m$-norm of $C_{0}^{m}\left(\mathbf{R}^{k} ; E\right)$ is "pushed forward" on $\Gamma_{0}^{m}(\Delta ; \mathcal{E})$.

We write "global" $m$-seminorms using a partition of unity $\left\{\psi_{i}\right\}_{i}$ subordinated to an open covering $\left\{\Delta_{i}\right\}_{i}$ of a compact $Q \subset \Omega$, i.e.

$$
\|\phi\|_{m, Q}=\sum_{i}\left\|\psi_{i} \phi\right\|_{\Gamma^{m}\left(\Delta_{i}, \mathcal{E}\right)} .
$$

One can show that different coverings and partitions of unity define equivalent $m$-seminorms.

Another way to define seminorms is to use covariant derivatives $\nabla_{\xi}$ on the bundle $(\mathcal{E}, \Omega)(\xi$ is a tangent vector field on $\Omega)$. Choosing a collection of vector fields $\xi_{1}, \ldots, \xi_{m}$ on $Q$ s.t. $\xi_{i}(a) \neq 0 \forall a \in Q$, we set

$$
\|\phi\|_{m, Q}=\sum_{0 \leq k \leq m} \sup _{x \in Q}\left\|\nabla_{\xi_{1}} \cdots \nabla_{\xi_{k}}(\phi)(x)\right\|_{E_{x}} .
$$

Here $\|\cdot\|_{E_{x}}$ means norm (metric) on the fibre $E_{x}$.

Once again different choices of vector fields give equivalent seminorms, and those are equivalent to (5.3.1).

Let us describe explicitly covariant derivatives in our setting, as we shall need them later on. We denote by $\partial_{1}, \ldots, \partial_{n}$ the basic partial derivatives on $\mathbf{R}^{n}$ restricted on $\Omega$, considered as elements (sections) of the trivial bundle $\Omega \times \mathbf{C}^{n}$. They form a basic of free generators of the $C^{m}(\Omega)$-module $\Gamma^{m}\left(\Omega \times \mathbf{C}^{n}\right)$ i.e. any $\phi \in$ $\Gamma^{m}\left(\Omega \times \mathbf{C}^{n}\right)$ splits uniquely into a linear combination $\sum_{1}^{n} c_{j}(x) \partial_{j}$, with $c_{j} \in C^{m}(\Omega)$. Then

$$
\nabla_{\xi}(\phi)=\sum_{1}^{n} \partial_{\xi}\left(c_{j}\right) \partial_{j}, \quad \xi \in \Gamma(\Omega ; \tau),
$$

where $\partial_{\xi}$ denotes a Lie derivative of a function $c_{j}$ along $\xi$. Taking tangential or normal components of $\nabla_{\xi}(\phi)$ for $\phi \in \Gamma^{m}(\Omega, \tau)$ or $\Gamma^{m}(\Omega, \mathcal{N})$ respectively, we get covariant derivatives on the tangent and normal bundles $\tau$ and $\mathcal{N}$, which then can be extended to all "tensor-bundles" $\varepsilon_{k, l}$ and $\mathcal{E}^{(r)}$. Notice that for $\mathcal{E}^{(r)}$-sections, realized as multilinear forms, the covariant derivative is given by

$$
\begin{array}{r}
\left(\nabla_{\xi} \phi\right)\left(x ; \eta_{1}, \ldots, \eta_{r}\right)=\nabla_{\xi}\left[\phi\left(x ; \eta_{1}, \ldots, \eta_{r}\right)\right]-\sum_{i=1}^{r} \phi\left(x ; \cdots \nabla_{\xi}\left(\eta_{i}\right) \cdots\right), \\
x \in \Omega, \eta_{1}, \ldots, \eta_{r} \in \Gamma\left(\Omega ; \mathbf{C}^{n}\right) .
\end{array}
$$

In the case when $\Omega$ is an orbit of a compact Lie group $U$, acting on $\mathbf{R}^{n}$, we shall need yet another characterization of $\Gamma^{m}(\Omega, \mathcal{E} \otimes B)$ and $m$-seminorms. Any such $U$ acts smoothly on all the above vector bundles $\mathcal{E}=\mathcal{E}_{k, l} ; \mathcal{E}^{(r)}$ etc. and consequently on the spaces of cross-sections on $\mathcal{E}$ by the induced representation $L$. The infinitesimal generators of $L$, elements of the Lie algebra $\mathfrak{H}$ of $U$, are given by certain 1st order differential operators.

We fix a basis $\left\{h_{1}, \ldots, h_{k}\right\}$ in $\mathfrak{H}(k=\operatorname{dim} \mathfrak{H})$ and consider corresponding differential operators and all their (noncommutative) polynomials, elements of the universal enveloping algebra $\mathfrak{U}(\mathfrak{H})$. These operators will play the role of partial derivatives in the Euclidean case. The space $\Gamma^{m}(\Omega ; \mathcal{E} \otimes B)$ will be characterized in terms of smooth vectors of the induced representation of $U$ and the $m$-norm will be defined using the above basis $\left(h_{1}, \ldots, h_{k}\right)$. 
Let us first recall the definition of $m$-smooth vectors for a general Banach representation $R$ of a Lie group. Let $u \mapsto R_{u}$ be a representation of $U$ on a Banach space $B$. A vector $\varsigma \in B$ is called $m$-smooth if the function $u \mapsto R_{u}(\varsigma)$ from $U$ to $B$ is $m$-smooth. We denote by $B_{m}$ the subset of all $m$-smooth vectors in $B$. It is well known that each $B_{m}$ is a dense $U$-invariant subspace of $B$, the spaces $B_{m}(m=0,1, \ldots)$ form a decreasing chain $B=B_{0} \supset B_{1} \supseteq B_{2} \supseteq \ldots$, and their intersection $B_{\infty}=\bigcap_{m=1}^{\infty} B_{m}$ known as the Garding space of the representation is also dense in $B$. Elements of the Lie algebra $\mathfrak{H}$ act on $B$ by infinitesimal operators

$$
R_{h}(\varsigma)=\left.\frac{d}{d t}\right|_{0} R_{\exp t h}(\varsigma) \quad\left(\varsigma \in B_{1}, h \in \mathfrak{H}\right)
$$

Obviously $R_{h}\left(B_{j}\right) \subset B_{j-1} \forall j \geq 1, h \in \mathfrak{H}$. Moreover, each element $p=p\left(h_{1}, \ldots, h_{k}\right)$ of the universal enveloping algebra $\mathfrak{U}(\mathfrak{H})$ maps $B_{j}$ into $B_{j-\operatorname{deg} p}$ provided $j \geq \operatorname{deg} p$.

Let us fix a basis $\left\{h_{1}, \ldots, h_{k}\right\}$ in $\mathfrak{H}$. For a multi-index $\nu=\left(\nu_{1}, \ldots, \nu_{k}\right)$ we denote by $h^{\nu}$ the monomial $\prod_{1}^{k} h_{i}^{\nu_{i}} \in \mathfrak{U}(\mathfrak{H})$. Though the elements $\left\{h_{1}, \ldots, h_{k}\right\} \subset \mathfrak{H}$ may not commute they satisfy the commutation relation $h_{i} h_{j}-h_{j} h_{i}=\left[h_{i}, h_{j}\right]$ and by the well-known Poincare-Birkhoff-Witt Theorem [23, Chapter 3] any element $p \in \mathfrak{U}(\mathfrak{H})$ is uniquely represented as a linear combination

$$
p=\sum_{|\nu| \leq m} c_{\nu} h^{\nu} \quad(m=\operatorname{deg} p) .
$$

We define the "abstract" $m$-norm on the space $B_{m}$ as

$$
\|\zeta\|_{m}=\sum_{|\nu| \leq m}\left\|R_{h^{\nu}}(\zeta)\right\|
$$

Since all operators $R_{h}(h \in \mathfrak{H})$ are closed as generators of strongly continuous groups one can show that $B_{m}$ is a Banach space with respect to the $m$-norm. It is invariant under the representation $R$ and the restriction $u \rightarrow R_{u} \mid B_{m}$ is a continuous (in $m$-norm) Banach representation of $U$.

By its definition (5.3.4) the $m$-norm depends upon a particular choice of basis $\left\{h_{1}, \ldots, h_{k}\right\}$ in $\mathfrak{H}$. But in fact one easily shows using the Poincare-Birkhoff-Witt Theorem, that different bases give equivalent $m$-norms.

Now we shall describe explicitly infinitesimal generators $L_{h}(h \in \mathfrak{H}), m$-smooth vectors and $m$-norms for induced representations $L$.

Let $(\mathcal{E}, \Omega)$ be a smooth $U$-homogeneous finite dimensional vector bundle with fibres $\left\{E_{a} \mid a \in \Omega\right\}$ and a cocycle $\tau(a, u)$,

$$
\tau(a, u): E_{a} \rightarrow E_{a^{u}} \quad(a \in \Omega ; u \in U) .
$$

We consider a Banach space $B$ and define a smooth Banach vector bundle $(\mathcal{E} \otimes B, \Omega)$, the action of $U$ on $B$ being trivial.

Let $D=\Gamma(\Omega ; \mathcal{E} \otimes B)$ denote the space of all bounded continuous sections of $(\mathcal{E} \otimes B, \Omega)$ equipped with $L^{\infty}$-norm.

The action of $U$ on $(\mathcal{E} \otimes B, \Omega)$ defines an induced representation on the space $D$,

$$
\left(L_{u} f\right)(a)=\left(\tau(a, u)^{-1} \otimes \mathbf{1}\right)\left[f\left(a^{u}\right)\right] \quad(u \in U, f \in D),
$$

where 1 stands for the trivial representation of $U$ on $B$. The representation $L$ is a tensor product of the induced representation on the space $\Gamma(\Omega ; \mathcal{E}$ ) (which will also 
be denoted by $L$, if it does not cause confusion) and the trivial representation on $B$. Indeed, the space $D$ decomposes into the product

$$
D=\Gamma(\Omega ; \mathcal{E} \otimes B)=\Gamma(\Omega, \mathcal{E}) \otimes^{\prime} B
$$

with a uniform $\left(L^{\infty}\right)$ cross-norm,

$$
\left\|\sum_{j} f_{j} \otimes b_{j}\right\|=\sup _{x \in \Omega}\left\|\sum_{j} f_{j}(x) b_{j}\right\| \quad\left(f_{j} \in \Gamma(\Omega ; \varepsilon), b_{j} \in B\right),
$$

i.e. $D$ coincides with the closure of all linear combinations $\sum f_{j} \otimes b_{j}$ in the norm (5.3.6).

The infinitesimal operators $L_{h}(h \in \mathfrak{H})$ of the induced representation $L$ are first order differential operators on the bundle $(\mathcal{E}, \Omega)$ (cf. [25, Chapter 1$])$, respectively elements $p \in \mathfrak{A}(\mathfrak{H})(\operatorname{deg} p=r)$ define $r$ th order differential operators. Let us notice that in the case of the trivial bundle $\mathcal{E}=\Omega \times E$ and the trivial cocycle $(\tau(x, u)=1 \quad \forall x, u)$, the induced representation has a form

$$
\left(L_{u} f\right)(x)=f\left(x^{u}\right), \quad f \in \Gamma(\Omega, \mathcal{E})=C(\Omega ; E) .
$$

Hence infinitesimal operators $L_{h}(h \in \mathfrak{H})$ are differentiations along $U$-invariant tangent vector fields $\xi=\xi(h) \in \Gamma^{\infty}(\Omega ; \tau)$, the images of elements $h \in \mathfrak{H}$ in $\Gamma^{\infty}(\Omega, \tau)$

$$
\left(L_{h} f\right)(x)=\left(\partial_{\xi(h)} f\right)(x)=\left.\frac{d}{d t}\right|_{0} f\left(x^{\exp t h}\right) .
$$

We remind the reader that the action of $U$ on $\Omega$ defines a homomorphism of $\mathfrak{H}$ into the Lie algebra $\Gamma^{\infty}(\Omega, \tau)$.

When the bundle $\mathcal{E}=\Omega \times E$ is trivial but the cocycle $\tau(x, u)$ is not we get

$$
\left(L_{h} f\right)(x)=\left(\partial_{\xi(h)} f\right)(x)+B(h, x) f(x) .
$$

Here the first-order term is a tangential derivative $\partial_{\xi(h)}$ and the zero-order term is a multiplication with $\operatorname{End}(E)$-valued $C^{\infty}$-function $B(h, \cdot)$, which depends on $h \in \mathfrak{H}$ and the cocycle $\tau(x, u)$. Finally if both $(\mathcal{E}, \Omega)$ and the cocycle $\tau(x, u)$ are nontrivial, the "tangential derivative" $\partial_{\xi}$ along $\xi=\xi(h) \in \Gamma^{\infty}(\Omega ; \tau)$ is replaced in (5.3.8) by a covariant derivative $\nabla_{\xi}$ on the bundle $\mathcal{E}$, i.e. (5.3.8) takes a form

$$
\left(L_{h} \phi\right)(x)=\left(\nabla_{\xi(h)} \phi\right)(x)+B(h, x) \phi(x), \quad \phi \in \Gamma^{1}(\Omega, \mathcal{E}) .
$$

The following lemma gives a characterization of $\Gamma^{m}(\Omega ; \mathcal{E} \otimes B)$ and $m$-norms (5.3.1), (5.3.2) in terms of $L$.

LEMMA 5. Let $(\mathcal{E}, \Omega)$ be a smooth finite dimensional $U$-homogeneous vector bundle over $\Omega$. The space of smooth sections $\Gamma^{m}(\Omega ; \mathcal{E} \otimes B)$ is equal to the space $D_{m}$ of all $m$-smooth vectors of the induced representation $L$ on the space of continuous sections $D=\Gamma(\Omega ; \mathcal{E} \otimes B)$. The $m$-norms on $\Gamma^{m}(\Omega ; \mathcal{E} \otimes B)$ defined by (5.3.1), (5.3.2) and by $L,(5.3 .4)$, are equivalent.

The proof of Lemma 5 is fairly standard, but for convenience we include it in Appendix A.

5.4. After all necessary preparations in the preceding sections (5.2)-(5.3) we are able to start the reduction procedure of the main problem. The first step is 
to reduce the problem from the space of $m$-smooth $B$-valued functions on $\mathbf{R}^{n}$ to "jet-functions" on $\Omega$.

Precisely, let $\Omega$ be a closed subset of $\mathbf{R}^{n}, I_{0}(\Omega, B)$ denote the (nonclosed) submodule of $A=C^{m}\left(\mathbf{R}^{n} ; B\right)$, whose elements, viewed as $B$-valued functions on $\mathbf{R}^{n}$, vanish at a neighbourhood of $\Omega$, and let $I(\Omega, B)$ be the closure of $I_{0}(\Omega ; B)$ in $A$, the minimal $C^{m}$-module with "hull $\Omega$ ".

Notice that for closed subset $\Omega \subset \mathbf{R}^{n}$, the submodule $\mathcal{M}=I(\Omega ; B)$ of $A$ has the "Whitney-type" characterization

$$
I(\Omega ; B)=\left\{f \in C^{m}\left(\mathbf{R}^{n} ; B\right) ; J^{m}(f, a)=0 \forall a \in \Omega\right\} .
$$

Indeed, the localizations of $\mathcal{M}$ are closed,

$$
\mathcal{M}(a)=0 \quad \forall a \in \Omega \quad \text { and } \quad \mathcal{M}(a)=J^{m} \otimes B \quad \forall a \notin \Omega .
$$

The first equality in (5.4.2) is obvious, while the second follows from the existence for any point $a \notin \Omega$ and a tuple $\left(b_{\nu}\right)_{|\nu| \leq m} \in J^{m} \otimes B$ of a smooth function $f \in$ $C^{m}\left(\mathbf{R}^{n} ; B\right)$ with the given jet $\left\{\left(\partial^{\nu} f\right)(a)=b_{\nu}\right\}_{|\nu| \leq m}$ at $\{a\}$, and such that $f$ vanishes at a neighborhood of $\Omega$.

As we mentioned earlier, the "finite dimensional" argument of Whitney's theorem (see [16, Chapter 2]) applies to any module $\mathcal{M}$ with closed localizations $\mathcal{M}(x)\left(x \in \mathbf{R}^{n}\right)$. So we get the equality $\overline{\mathcal{M}}=\tilde{\mathcal{M}}$, which in our case has the form (5.4.1).

We shall consider a quotient space $A / I(\Omega ; B)$. Any element $f \in A / I(\Omega ; B)$ can be identified with a tuple of partial derivatives restricted on $\Omega$,

$$
f_{\nu}(a)=\partial^{\nu} f|\Omega, \quad a \in \Omega,| \nu \mid \leq m .
$$

From Taylor's Theorem it follows that $B$-valued continuous functions $\left\{f_{\nu} \in\right.$ $C(\Omega, B),|\nu| \leq m\}$ satisfy the relations

$$
f_{\nu}(x)-\sum_{|\mu| \leq m-|\nu|} \frac{1}{\mu !} f_{\nu+\mu}(a)(x-a)^{\mu}=o\left(|x-a|^{m-|\nu|}\right) \quad \forall x, a \in \Omega,|\nu| \leq m
$$

Following [16], we call any tuple $F=\left(f_{\nu}\right)_{|\nu| \leq m}$ of continuous $B$-valued functions $\left\{f_{\nu}\right\}$ on a closed set $\Omega$ satisfying (5.4.4) a Whitney function on $\Omega$. The left-hand side of (5.4.4) is called a $\nu$-remainder of $F$ and denoted by $\left(R^{\nu} F\right)(x, a)$.

On the space $\mathbf{C}^{m}(\Omega ; B)$ of all Whitney functions on $\Omega$ one introduces a sequence of seminorms (see [16, Chapter 2])

$$
\|F\|_{m, Q}^{\prime}=\sum_{|\nu| \leq m}\left\|f_{\nu}\right\|_{L^{\infty}(Q)}+\sup \left\{\frac{\left\|\left(R^{\nu} F\right)(x, a)\right\|}{|x-a|^{m-\nu}} ; x, a \in Q\right\}
$$

where $Q$ runs over all compact subsets of $\Omega$. If $\Omega$ itself is compact, then $Q=\Omega$ in (5.4.5) defines as usual a Banach norm $\|F\|_{m}^{\prime}$ on $\mathbf{C}^{m}(\Omega, B)$.

We have already seen that any $m$-smooth function $f \in C^{m}\left(\mathbf{R}^{n} ; B\right)$ gives rise to a Whitney function $F=\left(f_{\nu}\right)_{|\nu| \leq m}$ by $(5.4 .3)$.

Conversely, in the scalar $(B=\mathbf{C})$ and finite-dimensional $(\operatorname{dim} B<\infty)$ cases the well-known Extension Theorem of Whitney asserts that any Whitney function $F=\left(f_{\nu}\right)_{|\nu| \leq m}$ is obtained from some $f \in C^{m}\left(\mathbf{R}^{n} ; B\right)$. Moreover, the "extension" $F \rightarrow f$ can be made a bounded linear operator. Precisely, 
PROPOSITION 8. There exists a linear operator $W: \mathbf{C}^{m}(\Omega ; B) \rightarrow C^{m}\left(\mathbf{R}^{n} ; B\right)$, s.t.

(1) $\left.\partial^{\nu}(W F)\right|_{\Omega}=f_{\nu} \forall|\nu| \leq m$,

(2) $\|W F\|_{m, Q} \leq C\|F\|_{m, Q}^{\prime} \forall F \in \mathbf{C}^{m}(\Omega ; B), Q \subset \Omega$.

The "finite dimensional" argument of this theorem (cf. [16, Chapter 1]) carries over verbatim to Banach vector-valued functions. Thus we get an isomorphism

$$
C^{m}\left(\mathbf{R}^{n} ; B\right) / I(\Omega ; B) \cong \mathbf{C}^{m}(\Omega ; B),
$$

for any closed subset $\Omega \subset \mathbf{R}^{n}$. Let us notice that the isomorphism (5.4.6) is topological.

Now we shall assume that $\Omega$ is a smooth submanifold of $\mathbf{R}^{n}$. The following important theorem gives a characterization of the quotient space $A / I(\Omega, B)$, equivalently the space of Whitney functions $\mathbf{C}^{m}(\Omega, B)$, for closed submanifolds $\Omega \subset \mathbf{R}^{n}$, in terms of normal bundles over $\Omega$.

THEOREM 3. (I) If $\Omega$ is a closed submanifold of $\mathbf{R}^{n}$, then there is a natural isomorphism of the spaces

$$
\mathbf{C}^{m}(\Omega ; B)=A / I(\Omega ; B) \cong \bigoplus_{l=0}^{m} \Gamma^{m-l}\left(\Omega ; \mathcal{E}_{l} \otimes B\right),
$$

where $\mathcal{E}_{l}=\mathcal{E}_{0, l}$ denotes the lth symmetric tensor power of the normal bundle $\mathcal{E}_{l}=$ $S_{l}(\mathcal{N})$ and $\Gamma^{k}\left(\Omega ; \mathcal{E}_{l} \otimes B\right)$ the space of all $k$-smooth sections of the bundle $\left(\mathcal{E}_{l} \otimes B, \Omega\right)$.

(II) Moreover, if $\Omega$ is an orbit of a Lie group $U$ acting smoothly on $\Omega$ and on the normal bundle $(\Omega, \mathcal{N})$, then the isomorphism (5.4.7) is topological, i.e. the sum of $(m-l)$-norms (5.3.4) on the spaces $\Gamma^{m-l}\left(\Omega, \mathcal{E}_{\rho} \otimes B\right)$ is equivalent to the norm (5.4.5) on the space of Whitney functions, the latter in its turn being equivalent to ordinary (without the "remainder") m-norm,

$$
\|F\|_{m, Q}^{\prime} \sim\|F\|_{m, Q}=\sum_{|\nu| \leq m}\left\|f_{\nu}\right\|_{L^{\infty}(Q)} .
$$

For convenience the proof of Theorem 3 will be given in Appendix B. Here we shall only describe explicitly the isomorphism (5.4.7) and mention some important facts derived in the argument.

Given a jet-function $\left\{f_{\nu}(x)\right\}_{|\nu| \leq m}$ on $\Omega$ we construct a tuple of symmetric $r$ forms $\phi^{(r)} \in \Gamma\left(\Omega ; \mathcal{E}^{(r)} \otimes B\right), r=0,1, \ldots, m$, whose "matrix entries" are functions $\left(f_{\nu}\right)_{|\nu|=r}$, i.e. a symmetric multilinear form $\phi^{(r)}$ evaluated on a tuple of basic vector fields

is equal to

$$
\underbrace{\partial_{1}, \ldots, \partial_{1}}_{\nu_{1} \text {-times }} ; \underbrace{\partial_{2}, \ldots, \partial_{2}}_{\nu_{2} \text {-times }} ; \ldots ; \underbrace{\partial_{n}, \ldots, \partial_{n}}_{\nu_{n} \text {-times }}, \quad \nu=\left(\nu_{1}, \nu_{2}, \ldots, \nu_{n}\right),
$$

$$
\phi^{(r)}\left(x ; \partial_{1}, \ldots, \partial_{1} ; \ldots ; \partial_{n}, \ldots\right)=f_{\nu}(x), \quad x \in \Omega .
$$

By multilinearity and symmetry $\phi^{(r)}$ extends to all other tuples $\xi_{1}, \ldots, \xi_{r} ; \xi_{i}=$ $\sum_{j=1}^{n} c_{i j}(x) \partial_{j} \in \Gamma\left(\Omega \times \mathbf{C}^{n}\right)$, hence defines an element of $\Gamma\left(\Omega ; \mathcal{E}^{(r)} \otimes B\right)$.

It turns out that the forms $\left\{\phi^{(r)}\right\}_{r=0}^{m}$ constructed from a jet $\left(f_{\nu}\right)_{|\nu| \leq m}$ are $(m-r)$ smooth,

$$
\phi^{(r)} \in \Gamma^{m-r}\left(\Omega ; \mathcal{E}^{(r)} \otimes B\right),
$$


and related in the following way; for any tangent field $\xi \in \Gamma(\Omega ; \tau)$ and a tuple $\eta_{1}, \ldots, \eta_{r} \in \Gamma\left(\Omega \times \mathbf{C}^{n}\right)$,

$$
\nabla_{\xi}\left[\phi^{(r)}\right]\left(\eta_{1}, \ldots, \eta_{r}\right)=\phi^{(r+1)}\left(\xi ; \eta_{1}, \ldots, \eta_{r}\right), \quad r=0,1, \ldots, m-1 .
$$

In differential-geometric terms the right-hand side of (5.4.10) means the contraction $i_{\xi}$ of the tensor-field $\phi^{(r+1)}$. So (5.4.10) can also be stated as the equality $\nabla_{\xi} \phi^{(r)}=$ $i_{\xi} \phi^{(r+1)}$.

Expanding $\phi^{(r)}$ into the sum of $(k, l)$-components $\phi^{(r)}=\bigoplus_{k+l=r} \phi_{k, l}$ according to (5.2.4) and using (5.4.9), (5.4.10) one can show that all $\left\{\phi_{k, l}\right\}_{k \geq 0}$ are uniquely determined by the normal components $\left\{\phi_{0, l}\right\}_{l=0}^{m}$ and $\left\{\phi_{0, l}\right\}$ are $(m-l)$-smooth (see Appendix B for details).

5.5. Now we return to the problem posed at the beginning of the section, i.e., the study of minimal ideals $I(\omega)$ of the algebra $A=C_{0}^{m}\left(\mathbf{R}^{n} ; B\right)$ with hull $\omega \subset \mathbf{R}^{n} \times X$, an orbit of the compact group $U$ acting on $\mathbf{R}^{n}$ and $B$.

Denote by $\Omega=p(\omega)$ the projection of $\omega$ on $\mathbf{R}^{n}$ (the orbit of $U$ in $\mathbf{R}^{n}$ ) and for any $a \in \Omega$ denote by $\omega_{a}$ the fibre $p^{-1}(a) \cap \omega$, identified with the orbit in $X$ of the stabilizer $U_{a}$ of $a \in \Omega$. We have to prove the equality

$$
I(\omega)=\left\{f \in \mathcal{A} \mid J^{m}(f, a) \in J^{m} \otimes I\left(\omega_{a}\right) \forall a \in \Omega\right\},
$$

where $I\left(\omega_{a}\right)$ is the minimal closed ideal of $B$ with the hull $\omega_{a} \subset X$. We denote by $\tilde{I}(\omega)$ the right-hand side of $(5.5 .1)$.

The results of $\S \S 5.3-5.4$ will be used in order to reformulate the problem in terms of smooth sections over $\Omega$. For this it is more convenient to consider nonclosed minimal ideals $I_{0}\left(\omega_{a}\right)(a \in \Omega)$ of the algebra $B$, and $I_{0}(\omega)$ of the algebra $A$. By definition they consist of all functors vanishing at a neighborhood of $\omega_{a}$ and $\omega$. The minimal ideals $I\left(\omega_{a}\right) \subset B$ and $I(\omega) \subset A$ are closures of $I_{0}\left(\omega_{a}\right)$ and $I_{0}(\omega)$ in $B$ and $A$ respectively.

The corresponding equality for nonclosed ideals should be

$$
I_{0}(\omega)=\left\{f \in \mathcal{A} \mid J^{m}(f, a) \in J^{m} \otimes I_{0}\left(\omega_{a}\right) \forall a \in \Omega\right\}
$$

which is obviously false as the left-hand side is included in the right, but not vice versa. However both sides of (5.5.2) become equal after factorization modulo the minimal ideal $I(\Omega ; B)$ (notice that both sides of $(5.5 .1)$ contain $I(\Omega ; B)$ ). Precisely, the quotient $I_{0}(\omega) / I(\Omega ; B)$ is equal to a subspace $\mathcal{M}_{0}=\left\{F \in \mathbf{C}^{m}(\Omega ; B) \mid F(a) \in\right.$ $\left.J^{m} \otimes I_{0}\left(\omega_{a}\right), a \in \Omega\right\}$ in the space of Whitney functions.

Another way to state the equality

$$
I_{0}(\omega) / I(\Omega ; B)=M_{0}
$$

is to say that each Whitney function $F=\left(f_{\nu}\right)_{\nu \leq m}$ on $\Omega$ with the property $f_{\nu}(a) \in$ $I_{0}\left(\omega_{a}\right) \forall a \in \Omega$, extends to a smooth function $f(x) \in C^{m}\left(\mathbf{R}^{n} ; B\right)$, vanishing at a neighborhood of $\omega$. In such a form (5.5.3) follows from the explicit construction of an extension operator $W: F \rightarrow f$ in Whitney's Theorem [16, Chapter 1, §3]. Namely, the value of $f=W F$ at $x$ is obtained as a linear combination of "Taylor series" $\sum(1 / \nu !) f_{\nu}(a)(x-a)^{\nu}$ at "nearby" points $\{a\} \subset \Omega$. Taking $x$ sufficiently close to $\Omega$ all Taylor coefficients $\left\{f_{\nu}(a)\right\}_{\nu \leq m}$ at "nearby" $a$ 's have a common open "hull" $\Delta \subset X$ (a neighborhood of $\omega_{a}$ ), i.e. $\left\{f_{\nu}(a)\right\}_{\nu}$, consequently the value of $f$ at $x, f(x) \in B$, belong to the minimal ideal $I(\Delta)$ of $B$. The "size" of $\Delta$ depends only 
on the distance between $x$ and $\Omega$, hence the function $f(x)=W F \in \mathcal{A}$ vanishes at a neighborhood of $\omega$.

After (5.5.3) the problem can be reformulated in terms of modules in the space of Whitney functions on $\Omega$. Along with $\mathcal{M}_{0}$ we consider a closed submodule

$$
\mathcal{M}=\left\{F \in \mathbf{C}^{m}(\Omega ; B) \mid F(a) \in J^{m} \otimes I\left(\omega_{a}\right) \forall a \in \Omega\right\} .
$$

Then (5.5.3) is equivalent to

$$
\mathcal{M}=\bar{M}_{0} \quad\left(\text { the closure in } \mathbf{C}^{m}(\Omega ; B)\right) .
$$

Next we shall apply the isomorphism (5.4.7) of Theorem 3,

$$
\mathbf{C}^{m}(\Omega ; B) \cong \bigoplus_{l=0}^{m} \Gamma^{m-l}\left(\Omega ; \mathcal{E}_{l} \otimes B\right), \quad \mathcal{E}_{l}=S_{l}(\mathcal{N}),
$$

to write $\mathcal{M}_{0}$ and $\mathcal{M}$ as direct sums of submodules in the spaces of smooth sections.

LEMMA 6. (1) The images of modules $\mathcal{M}_{0}$ and $\mathcal{M}$ under the isomorphism (5.4.7) decompose into the direct sum

$$
\mathcal{M}_{0}=\bigoplus_{l=0}^{m} \mathcal{M}_{0, l}, \quad \mathcal{M}=\bigoplus_{l=0}^{m} \mathcal{M}_{l}
$$

where $\mathcal{M}_{0, l}, \mathcal{M}_{l} \subset \Gamma^{m-l}\left(\Omega ; \mathcal{E}_{l} \otimes B\right)$.

(2) The components $\mathcal{M}_{0, l}$ and $\mathcal{M}_{l}(l=0, \ldots, m)$ are characterized as

$$
\begin{aligned}
\mathcal{M}_{0, l} & =\left\{\phi \in \Gamma^{m-l}\left(\Omega ; \mathcal{E}_{l} \otimes B\right) \mid \phi(a) \in S_{l}\left(N_{a}\right) \otimes I_{0}\left(\omega_{a}\right), \forall a \in \Omega\right\}, \\
\mathcal{M}_{l} & =\left\{\phi \in \Gamma^{m-l}\left(\Omega ; \mathcal{E}_{l} \otimes B\right) \mid\left(L^{\nu} \phi\right)(a) \in S_{l}\left(N_{a}\right) \otimes I\left(\omega_{a}\right),\right. \\
& \forall a \in \Omega,|\nu| \leq m-l\} .
\end{aligned}
$$

Here $L^{\nu}=L_{h} \nu$ are differential operators of the induced representation $L$ on the bundle $\left(\mathcal{E}_{l} \otimes B ; \Omega\right)$ and $L_{h}, h \in \mathfrak{H}$, are given by (5.3.9).

ProOF. To check the first equality (5.5.5) we observe that the left-hand side is included in the right-hand side. Indeed for $F \in \mathcal{M}_{0}$ local $B$-values of its coefficients $f_{\nu}(a) \in I_{0}\left(\omega_{a}\right) \forall|\nu| \leq m$, hence forms $\left\{\phi^{(r)}(a)\right\}_{r=0}^{m}$ with matrix-entries $\left\{f_{\nu}(a)\right\}_{|\nu|=r}$ belong to $S_{r}\left(\mathbf{R}^{n}\right) \otimes I_{0}\left(\omega_{a}\right)$ (vanish at a neighbourhood of $\omega_{a}$ ) as well as their normal components, $\phi_{0, l}(a) \in S_{l}\left(N_{a}\right) \otimes I_{0}\left(\omega_{a}\right)$.

Conversely, if a tuple of $(0, l)$-forms has local values vanishing at a neighbourhood of $\omega_{a}, \phi_{0, l}(a) \in S_{l}\left(N_{a}\right) \otimes I_{0}\left(\omega_{a}\right)$, then one can show (cf. Appendix B) that the forms $\phi^{(r)}$ obtained from $\phi_{0, l}$ by iterated "differentiation (5.4.10)" will have the same property,

$$
\phi^{(r)}(a) \in S_{r}\left(\mathbf{R}^{n}\right) \otimes I_{0}\left(\omega_{a}\right), \quad \forall a \in \Omega .
$$

Then of course all matrix entries of $\phi^{(r)},\left\{f_{\nu}(a)\right\}_{\nu}$ belong to $I_{0}\left(\omega_{a}\right)$ and the jet $F=\left(f_{\nu}\right)_{|\nu| \leq m} \in \mathcal{M}_{0}$.

To prove the inclusion $\mathcal{M}_{l} \subset$ "right-hand side of (5.5.5)" in the second equality it suffices to check that for any $\phi^{(r)} \in \Gamma^{m-r}\left(\Omega ; \mathcal{E}^{(r)} \otimes B\right)$ obtained from a Whitney function $F \in \mathcal{M}$, one has

$$
\left(L^{\nu} \phi^{(r)}\right)(a) \in S_{r}\left(\mathbf{R}^{n}\right) \otimes I\left(\omega_{a}\right) \quad \forall a \in \Omega,|\nu| \leq m-r .
$$


But $F \in \mathcal{M}$ implies

$$
\phi^{(r)}\left(a ; \eta_{1}, \ldots, \eta_{r}\right) \in I\left(\omega_{a}\right) \quad \forall a \in \Omega, \forall \eta_{1}, \ldots, \eta_{r} \in C^{\infty}\left(\Omega ; \mathbf{R}^{n}\right) .
$$

By (5.4.12) an infinitesimal operator $L_{h}(h \in \mathfrak{H})$ maps $\phi^{(r)}$ into the form

$$
\begin{aligned}
\left(L_{h} \phi^{(r)}\right)\left(a ; \eta_{1}, \ldots, \eta_{r}\right)= & \phi^{(r+1)}\left(a ; \xi(h), \eta_{1}, \ldots, \eta_{r}\right) \\
& +B(h, a) \phi^{(r)}\left(a ; \eta_{1}, \ldots, \eta_{r}\right),
\end{aligned}
$$

whose $B$-value at $\{a\}$ is obviously in $I\left(\omega_{a}\right)$ for all $a \in \Omega, h \in \mathfrak{H}$ and all tuples, $\eta_{1}, \ldots, \eta_{r} \in C^{\infty}\left(\Omega ; \mathbf{R}^{n}\right)$. So

$$
\left(L_{h} \phi^{(r)}\right)(a) \in I\left(\omega_{a}\right) \otimes S_{r}\left(\mathbf{R}^{n}\right) \quad(a \in \Omega) .
$$

Iterating this formula $k$ times $(k \leq m-r)$ we get

$$
\left(L_{h_{1}} L_{h_{2}} \cdots L_{h_{k}} \phi^{(r)}\right)(a) \in I\left(\omega_{a}\right) \otimes S_{r}\left(\mathbf{R}^{n}\right) \quad(a \in \Omega), \forall h_{1} \cdots h_{k} \subset \mathfrak{H} .
$$

Conversely, if a form $\phi^{(r)} \in \Gamma^{m-r}\left(\Omega ; \mathcal{E}^{(r)} \otimes B\right)$ satisfies (5.5.6) with $k=0$, the coefficients $\left(f_{\nu}\right)_{|\nu|=r}$ of the form $\phi^{(r)}$ are obviously in $I\left(\omega_{\alpha}\right)$. Thus we get: a Whitney function $F \in \mathcal{M}$ if and only if

$$
\left(L^{\nu} \phi^{(r)}\right)(a) \in I\left(\omega_{a}\right) \otimes S_{r}\left(\mathbf{R}^{n}\right) \quad \forall a \in \Omega, 0 \leq r \leq m,|\nu| \leq m-r .
$$

Formula (5.5.5) follows when we restrict each form $\phi^{(r)}, r=0,1, \ldots, m$, to its normal component $\phi_{0, l}, l=r$.

5.6. The problem we have to deal with now can be reformulated as follows. Let $(\mathcal{E}, \Omega)$ be a finite dimensional smooth $U$-homogeneous vector bundle with fibres $\{E(a): a \in \Omega\}$ and a cocycle $\tau(a, u)(a \in \Omega, u \in U)$. Given a Banach space $B$ we consider a Banach vector bundle $(\mathcal{E} \otimes B, \Omega)$ with the trivial action of $U$ on the second factor. As above we denote by $L$ the induced representation of $U$ on the spaces of smooth sections $\Gamma^{m}(\Omega ; \mathcal{E}), \Gamma^{m}(\Omega ; \mathcal{E} \otimes B)(m=0,1, \ldots)$ and by $L_{h}(h \in \mathfrak{H})$ its infinitesimal (differential) operators.

Let us also assume that we are given a representation $R$ of $U$ on $B$ and a system of (nonclosed) subspaces of $B,\left\{I_{0}(a) \mid a \in \Omega\right\}$, with the property

$$
R_{u} I_{0}(a)=I_{0}\left(a^{u}\right) \quad \forall a \in \Omega, u \in U .
$$

In our case $I_{0}\left(\omega_{a}\right)$ will play the role of $I_{0}(a)$. We denote by $I(a)$ the closure of $I_{0}(a)$ in $B$ (in our case $I(a)=I\left(\omega_{a}\right)$ ). Then we also have

$$
R_{u} I(a)=I\left(a^{u}\right) \quad \forall a \in \Omega, u \in U .
$$

Given systems of subspaces $\left\{I_{0}(a)\right\}_{a \in \Omega}$ and $\{I(a)\}_{a \in \Omega}$ we can define a pair of submodules $\mathcal{M}_{0}$ and $\mathcal{M}$ in the space of smooth sections $\Gamma^{m}(\Omega ; \mathcal{E} \otimes B)$, similar to (5.5.5),

$$
\begin{aligned}
\mathcal{M}_{0} & =\left\{f \in \Gamma^{m}(\Omega ; \mathcal{E} \otimes B) \mid f(a) \in E(a) \otimes I_{0}(a) \forall a \in \Omega\right\}, \\
\mathcal{M} & =\left\{f \in \Gamma^{m}(\Omega ; \mathcal{E} \otimes B)\left|\left(L^{\nu} f\right)(a) \in E(a) \otimes I(a) \forall a \in \Omega,\right| \nu \mid \leq m\right\} .
\end{aligned}
$$

We have to prove the equality

$$
\mathcal{M}=\bar{M}_{0} \quad\left(\text { the closure in the } m \text {-norm on } \Gamma^{m}(\Omega ; \mathcal{E} \otimes B)\right) .
$$

For this we shall impose some conditions on the spaces $\left\{I_{0}(a) ; I(a)\right\}_{a \in \Omega}$ and the action of $U$ on $(\mathcal{E}, \Omega)$ and $B$, which eventually enable us to "localize" the problem 
to a single point $a \in \Omega$ and thus to solve it. Later we shall check these conditions in the case that we are mostly interested in, i.e., when $B=A_{\rho}(X)$ is a Fourier algebra on a compact group $X$ and $U$ acts by translations on $X$.

The conditions are:

(I) If a smooth section $f \in \mathcal{M}_{0} \subset \Gamma^{m}(\Omega ; \mathcal{E} \otimes B)$, then all "partial derivatives" $\left(L^{\nu} f\right)(|\nu| \leq m)$ satisfy

$$
\left(L^{\nu} f\right)(a) \in E(a) \otimes I(a) \quad \forall a \in \Omega
$$

i.e., any "derivative" $L^{\nu} f$ of an element $f \in \mathcal{M}_{0}$ is itself in the "M-type" subspace of the space $\Gamma^{m-|\nu|}(\Omega ; \mathcal{E} \otimes B)$.

Notice that instead of infinitesimal operators $L$ one can use covariant derivatives, $\nabla_{\xi_{1}}^{\nu_{1}} \cdots \nabla_{\xi_{r}}^{\nu_{r}}$, in (5.6.5), $\xi_{i}$ being tangent vector fields. We need property (I) in order to have the inclusion $\mathcal{M}_{0} \subset \mathcal{M}$.

(II) The subspace $I_{0}(a) \cap B_{m}$ is dense in the $m$-norm of $B_{m}$ in the space

$$
I(a)_{m}=\left\{b \in B_{m}\left|R^{\nu}(b) \in I(a) \forall\right| \nu \mid \leq m\right\},
$$

for all $a \in \Omega$. Here $R^{\nu}$ are products of infinitesimal operators $R_{h_{1}}^{\nu_{1}} \cdots R_{h_{k}}^{\nu_{1}}$ corresponding to elements $h_{1}, \ldots, h_{k} \subset \mathfrak{H}^{4}{ }^{4}$

(III) For any $a \in \Omega$ the subspace $I_{0}(a)$ is stable under the primary (isotypical) decomposition of the representation $v \rightarrow R_{v}$ of the stabilizer $U_{a}$ on $B$ (see $\S 1.2$ ), i.e., if $b \in I_{0}(a)$, then $P_{\sigma}(b) \in I_{0}(a) \forall \sigma \in \hat{U}_{a}$.

(IV) The submodule $\mathcal{M}_{0}$ is stable under the primary decomposition of the representation $L \otimes R$ of the group $U$, i.e., if $f \in \mathcal{M}_{0} \subset \Gamma^{m}(\Omega ; \mathcal{E} \times B)$, then $P_{\pi}(f) \in$ $\mathcal{M}_{0} \forall \pi \in \hat{U}$.

THEOREM 4. Let $(\mathcal{E}, \Omega)$ be a finite dimensional smooth $U$-homogeneous vector bundle, $R$ a representation of $U$ on a Banach space $B$ and $\left\{I_{0}(a), I(a)\right\}_{a \in \Omega}$ a system of subspaces of $B$ satisfying conditions (I)-(II). If the submodules $\mathcal{M}_{0}$ and $\mathcal{M}$ in the spaces of sections $\Gamma^{m}(\Omega ; \mathcal{E} \otimes B)$ are defined by (5.6.3), then

$$
\left.\mathcal{M}=\bar{M}_{0} \quad \text { (the closure in the m-norm of } \Gamma^{m}(\Omega ; \mathcal{E} \otimes B)\right) .
$$

Proof. First notice that the space $A=\Gamma^{m}(\Omega ; \mathcal{E} \otimes B)$ has a natural tensor product structure, $\mathcal{A}=\Gamma^{m}(\Omega ; \mathcal{E}) \otimes^{\prime} B$ with " $m$-uniform cross-norm",

$$
\left\|\sum_{j} f_{j} \otimes b_{j}\right\|_{m}=\sum_{|\nu| \leq m} \max \left\{\left\|\sum_{j} L^{\nu}\left(f_{j}\right)(x) \otimes b_{j}\right\| ; x \in \Omega\right\}
$$

$\left(f_{j} \in C^{m}(\Omega ; \mathcal{E}), b_{j} \in B\right)$. The group $U$ acts on $A$ by tensor product of the induced representation $L$ on $\Gamma^{m}(\Omega ; \mathcal{E})$ and the representation $R$ on $B$.

The submoudles $\mathcal{M}_{0}$ and $\mathcal{M}$ of $A$ are invariant under the representation $L \otimes^{\prime} R$ on $\Gamma^{m}(\Omega, \mathcal{E}) \otimes^{\prime} B$. So they decompose into the direct sum of their $\pi$-primary components $(\pi \in \hat{U})$. This is obvious for the closed $L \otimes R$-invariant subspace $\mathcal{M}$ of $A$ and for $\mathcal{M}_{0}$ follows from assumption (IV). Since $\mathcal{M}_{0}(\pi) \subset \mathcal{M}(\pi)$ it suffices to prove the equality

$$
\overline{\mathcal{M}_{0}(\pi)}=\mathcal{M}(\pi) \quad \text { for all } \pi \in \hat{U} \text {. }
$$

\footnotetext{
${ }^{4}$ The notation $I(a)_{m}$ has a slightly different meaning from that of $B_{m}$, as $I(a)$ is not $U$ invariant, but only " $U_{a}$-". However we consider $m$-smooth vectors of $I(a)$ with respect to the whole Lie algebra $\mathfrak{H}$ of $U$.
} 
Let us first prove it for the trivial representation $\pi=\mathbf{1} \in \hat{U}$. The 1-component $A(\mathbf{1})$ consists of all $U$-invariants of the representation $L \otimes R$, i.e.,

$$
f \in \mathcal{A}(\mathbf{1}) \Leftrightarrow(L \otimes R)_{u} f=f \quad \forall u \in U .
$$

Consequently, the 1-primary components of $\mathcal{M}$ and $\mathcal{M}_{0}$ consist of all $L \otimes R$-invariants of $\mathcal{M}$ and $\mathcal{M}_{0}$ respectively.

The invariance relation (5.6.7) implies that each section $f \in A(\mathbf{1})$ is uniquely determined by its value at a single point $a \in \Omega$ and the element $f(a) \in E(a) \otimes B$ is invariant under the representation $v \rightarrow \tau(a, v) \otimes R_{v}$ of the stabilizer $U_{a}$,

$$
\left(\tau\left(a, v^{-1}\right) \otimes R_{v}\right)[f(a)]=f(a) \quad \forall v \in U_{a} .
$$

We rewrite the invariance relation (5.6.7) in infinitesimal form, i.e., for elements $h$ of the Lie algebra $\mathfrak{H}$

$$
\left[\left(L_{h} \otimes I\right) f\right](a)=-\left(I \otimes R_{h}\right)(f(a)) \quad(h \in \mathfrak{H}) .
$$

Consequently,

$$
\left[\left(L^{\nu} \otimes I\right) f\right](a)=(-1)^{|\nu|}\left(I \otimes R^{\nu}\right)[f(a)] .
$$

The latter equality shows that the vectors $\{\bar{b}=f(a) \in E(a) \otimes B, f \in A(\mathbf{1})\}$ are $m$-smooth with respect to the rerpesentation $I \otimes R$ on $E(a) \otimes B$. Conversely, any $m$-smooth vector $\bar{b} \in E(a) \otimes B_{m}$, which satisfies (5.6.7) extends to a $U$-invariant $m$-smooth section $f(x) \in A(1)$ such that $f(a)=\bar{b}$. Namely,

$$
f(x)=\tau(a, u) \otimes R_{u}^{-1}(\bar{b}),
$$

where $x=a^{u}$. By (5.6.7) $f(x)$ is independent on a particular choice of an element $u \in U$, which takes $a$ to $x$. So (5.6.9) defines a continuous section $f(x)$ satisfying the invariance relation (5.6.6). To check that $f(x)$ is $m$-smooth let us compute its "partial derivatives" $\left\{L^{\nu} f\right\}_{\nu}$. Obviously, at the point $\{a\}$

$$
\left(L_{h}^{\nu} f\right)(a)=(-1)^{|\nu|} R_{h}^{\nu}(\bar{b}),
$$

exists for all $|\nu| \leq m$ and $h=\left(h_{1}, \ldots, h_{k}\right) \subset \mathfrak{H}$. Then for any other point $x \in \Omega$ we get

$$
\begin{aligned}
\left(L^{\nu} f\right)(x) & =\left(\tau(a, u) \otimes R_{u}^{-1}\right)\left[\left(L_{\mathrm{ad}_{u}^{-1}\left(h^{\nu}\right)} f\right)(a)\right] \\
& =\left(\tau(a, u) \otimes R_{u}^{-1}\right)(-1)^{|\nu|} \times \sum_{|\mu| \leq|\nu|} \alpha_{\nu, \mu}(u) R^{\mu}(\bar{b}) .
\end{aligned}
$$

The coefficients $\alpha_{\nu, \mu}(u)$ in the sum (5.6.11) are obtained from the expansion of an element $\operatorname{ad}_{u}^{-1}\left(h^{\nu}\right) \in \mathfrak{A}(\mathfrak{H})$ in the Poincaré -Birkhoff-Witt basis $\left\{h^{\mu}\right\}_{|\mu| \leq|\nu|}$, where $h=\left(h_{1}, \ldots, h_{k}\right)$ is a basis in $\mathfrak{H}$.

So a partial derivative $L^{\nu}(f)$ exists and is continuous for all $|\nu| \leq m$, i.e., $f \in$ $\Gamma^{m}(\Omega ; \mathcal{E} \otimes B)$. Thus we get the following characterization of $A(1)$ : a section $f \in$ $A(\mathbf{1})$ iff

$$
\begin{aligned}
& \text { (a) } \bar{b}=f(a) \in E(a) \otimes B_{m} \quad \forall a \in \Omega, \\
& \text { (b) }\left(\tau(a, u)^{-1} \otimes R_{u}\right)(\bar{b})=\bar{b} \quad \forall u \in U_{a} .
\end{aligned}
$$


Moreover, $m$-norms in the spaces $E(a) \otimes B_{m}$ and $A(\mathbf{1}) \subset \Gamma^{m}(\Omega ; \mathcal{E} \otimes B)$ are equivalent. Indeed,

$$
\left\|R^{\nu}(\bar{b})\right\|=\left\|\left(L^{\nu} f\right)(a)\right\| \leq \sup \left\{\left\|\left(L^{\nu} f\right)(x)\right\|: x \in \Omega\right\} .
$$

So $\|\bar{b}\|_{m} \leq\|f\|_{m}$. The inverse inequality follows from (5.6.11).

Conditions (5.6.12) yield a similar characterization of 1 -components $\mathcal{M}(\mathbf{1}) \subset \mathcal{M}$ and $\mathcal{M}_{0}(\mathbf{1}) \subset \mathcal{M}_{0}$. Namely, $f \in \mathcal{M}_{0}(\mathbf{1})$ iff

(a) $\bar{b}=f(a) \in E(a) \otimes\left(I_{0}(a) \cap B_{m}\right) \quad \forall a \in \Omega$,

(b) $\left(\tau(a, u)^{-1} \otimes R_{u}\right) \bar{b}=\bar{b} \quad \forall u \in U_{a}$.

Correspondingly, $f \in \mathcal{M}(\mathbf{1})$ iff

(a) $(\bar{b})=f(a) \in E(a) \otimes B_{m}$ and

$$
R^{\nu}(\bar{b}) \in E(a) \otimes\left(I(a) \cap B_{(m-|\nu|)}\right) \quad \forall|\nu| \leq m,
$$

(b) $\left(\tau(a, u)^{-1} \otimes R_{u}\right) \bar{b}=\bar{b} \quad \forall u \in U_{a}$.

Now let $\bar{b} \in E(a) \otimes B_{m}$ satisfy (5.6.14). By property (II) it can be approximated by $\bar{b}_{0} \in E(a) \otimes\left(I_{0}(a) \cap B_{m}\right)$, such that $\left\|b-b_{0}\right\|_{m}<\varepsilon$. Since any Banach representation of a compact group is bounded there exists a constant $c=c(R, m)$ such that

$$
\left\|\bar{b}-R_{u}\left(\bar{b}_{0}\right)\right\|_{m}=\left\|R_{u}\left(\bar{b}-\bar{b}_{0}\right)\right\|_{m} \leq c \varepsilon \quad \forall u \in U_{a} .
$$

Averaging the function $\bar{b}-R_{u}\left(\bar{b}_{0}\right)$ over $U_{a}$ we get

$$
\left\|\bar{b}-\bar{b}_{0}(\mathbf{1})\right\|_{m} \leq c \varepsilon
$$

where $\bar{b}_{0}(\mathbf{1})=\int_{U_{a}} R_{u}\left(\bar{b}_{0}\right) d u$ is the 1-component of $\bar{b}_{0}$. By (III) $\bar{b}_{0}(\mathbf{1})$ is in $E(a) \otimes$ $I_{0}(a)$ along with $\bar{b}_{0}$, and it satisfies the invariance relation $(5.6 .12)(\mathrm{b})$.

If $f(x)$ and $f_{0}(x)$ is a pair of continuous sections in $\mathcal{M}(\mathbf{1})$ and $\mathcal{M}_{0}(\mathbf{1})$ obtained from the local values $\bar{b}$ and $\bar{b}_{0}(\mathbf{1})$ by $(5.6 .9)$, then

$$
\left\|f-f_{0}\right\|_{m} \leq c^{\prime}\left\|b-\bar{b}_{0}(\mathbf{1})\right\|_{m} \leq c^{\prime} c \varepsilon .
$$

This proves the equality $\overline{\mathcal{M}_{0}(\mathbf{1})}=\mathcal{M}(\mathbf{1})$ for 1-primary components. In order to prove it for a nontrivial $\pi \in \hat{U}$ we shall realize the spaces $\mathcal{M}_{0}(\pi)$ and $\mathcal{M}(\pi)$ as 1-primary components of a certain finite extension of the bundle $\mathcal{E} \otimes B$.

Let $d=d(\pi)=d\left(\pi^{*}\right)$ be a degree of $\pi$. We consider a smooth vector bundle $\mathcal{E}^{\prime}=\mathcal{E} \otimes \mathbf{C}^{d}\left(\mathbf{C}^{d}\right.$ is identified with the trivial bundle $\left.\Omega \times \mathbf{C}^{d}\right)$ and a cocycle

$$
\tau^{\prime}(a, u)=\tau(a, u) \otimes \pi^{*}(u) \quad(a \in \Omega, u \in U) .
$$

As above we define a Banach vector bundle $\left(\mathcal{E}^{\prime} \otimes B, \Omega\right)$, the space of sections $A^{\prime}=\Gamma^{m}\left(\Omega ; \mathcal{E}^{\prime} \otimes B\right)$ and the submodules $\mathcal{M}_{0}^{\prime}$ and $\mathcal{M}^{\prime}$ of $A^{\prime}$.

Conditions (I) through (IV) are easily verified for the new representation $L^{\prime}$ and modules $\mathcal{M}^{\prime}$ and $\mathcal{M}_{0}^{\prime}$. Notice that the space $\Gamma^{m}\left(\Omega ; \mathcal{E}^{\prime}\right)=\Gamma^{m}(\Omega ; \mathcal{E}) \otimes \mathbf{C}^{d}$ and the representation $L^{\prime} \cong L \otimes \pi^{*}$.

Let $\mathcal{A}^{\prime}(\mathbf{1}), \mathcal{M}^{\prime}(\mathbf{1})$ and $\mathcal{M}_{0}^{\prime}(1)$ be 1-primary components of the representation $L^{\prime} \otimes R$ acting on the spaces $A^{\prime}, M^{\prime}$ and $\mathcal{M}_{0}^{\prime}$ respectively. Since $A^{\prime} \cong A \otimes \mathbf{C}^{d}$ we can attach to each section $f^{\prime} \in \mathcal{A}^{\prime}$ a tuple $\left(f_{1}, \ldots, f_{d}\right)$ of sections $f_{j} \in \mathcal{A}$. Let us define a continuous linear map $W: A^{\prime} \rightarrow \mathcal{A}$ as $W: f^{\prime} \rightarrow \sum_{j=1}^{d} f_{j} . W$ is easily verified 
to map $\mathcal{A}^{\prime}(\mathbf{1}), \mathcal{M}^{\prime}(\mathbf{1})$, and $\mathcal{M}_{0}^{\prime}(\mathbf{1})$ onto $\mathcal{A}(\pi), \mathcal{M}(\pi)$ and $\mathcal{M}_{0}(\pi)$ respectively. As we have already proved $\overline{\mathcal{M}}_{0}^{\prime}(\mathbf{1})=\mathcal{M}^{\prime}(\mathbf{1})$, then of course, $\overline{\mathcal{M}_{0}(\pi)}=\mathcal{M}(\pi) \forall \pi \in \hat{U}$, which implies $\overline{\mathcal{M}}_{0}=\mathcal{M}$. The theorem is proved.

5.7. In order to apply Theorem 4 to the proof of Lemma 3 we have only to check the above conditions (I)-(IV) in the case when $B=A_{\rho}(X)$ is the Fourier algebra on the compact abelian Lie group $X$ with a finite weight $\rho$ on the dual group $A=\hat{X}$. The representation $R$ of $U$ on $B$ is given by translations

$$
\left(R_{u} \hat{f}\right)(x)=\hat{f}(x+l(u)) \quad\left(f \in A_{\rho}(X), u \in U\right),
$$

where $l: U \rightarrow X$ is a group homomorphism.

We also assume that $U$ acts by affine transformations on $\mathbf{R}^{n}$. Then for any orbit $\omega \subset \mathbf{R}^{n} \times X$ with a projection $\Omega \subset \mathbf{R}^{n}$ and fibres $\omega_{a} \subset X(a \in \Omega)$, we shall define a system of subspaces $\left\{I_{0}(a)\right\}_{a \in \Omega}$ and $\{I(a)\}_{a \in \Omega}$ as nonclosed and closed minimal ideals of $B, I_{0}(a)=I_{0}\left(\omega_{a}\right), I(a)=I\left(\omega_{a}\right)$.

Each $\omega_{a} \subset X$ is an orbit of a stabilizer $U_{a}$ acting on $X$ by translations and a subgroup $Z=l\left(U_{a}\right) \subset X$ is independent of $a \in \Omega$. Therefore all $\omega_{a}(a \in \Omega)$ are cosets of the same (fixed) subgroup $Z \subset X$.

LEMMA 7. The system of subspaces $\left\{I_{0}\left(\omega_{a}\right), I\left(\omega_{a}\right)\right\}_{a \in \Omega}$ and the representation $R$ of the group $U$ on the space $B=\mathcal{A}_{\rho}(X)$ satisfy properties (I)-(IV).

ProOF. Properties (I), (III) and (IV) are easily verified. For instance, to check (I) we note that, if for some $f \in \Gamma^{m}(\Omega ; \mathcal{E} \otimes B)$ an element $\bar{b}=f(a) \in E(a) \otimes A_{\rho}(X)$ considered as a vector-function on $X$,

$$
\bar{b}(x)=\left(b_{1}(x), \ldots, b_{k}(x)\right), \quad b_{j}(x) \in A_{\rho}(X), k=\operatorname{dim} E(a),
$$

vanishes at a neighborhood $\Delta_{a}$ of $\omega_{a}\left(\Delta_{a} \subset X\right)$, then there exists a pair of neighborhoods $\Delta^{\prime}$, a neighborhood of $a$ in $\Omega$, and $\Delta^{\prime \prime}$ a neighborhood of $\omega_{a}$ in $X$, such that $\Delta^{\prime \prime}$ contains all $\omega_{x}\left(x \in \Delta^{\prime}\right)$, i.e., all elements $f(x)\left(x \in \Delta^{\prime}\right)$ satisfy

$$
f(x) \in E(x) \otimes I_{0}\left(\Delta^{\prime \prime}\right) \quad\left(x \in \Delta^{\prime}\right) .
$$

Then, of course, all covariant partial derivatives $\left(\nabla^{\nu} f\right)(a)$ "vanish on $\Delta^{\prime}$, i.e.,

$$
\left(\nabla^{\nu} f\right)(a) \in E(a) \otimes I_{0}\left(\omega_{a}\right) \quad \forall|\nu| \leq m .
$$

Consequently, $\left(L^{\nu} f\right)(a) \in E(a) \otimes I_{0}\left(\omega_{a}\right), \forall|\nu| \leq m$. Since the latter holds for any point $a \in \Omega$ we obtain property (I).

To check (III) we take an element $\hat{b} \in \mathcal{A}_{\rho}(X)$ that vanishes at a neighborhood $\Delta$ of a coset $\omega_{a}=Z+x$ and notice that there exists a $Z$-invariant neighborhood $\Delta^{\prime} \subset \Delta$ of $\omega_{a}$. Then all isotypical components $\hat{b}_{\chi}(\chi \in \hat{Z})$ will vanish on $\Delta^{\prime}$.

To check (IV) let us note that the module $\mathcal{M}_{0}$ in our case is a union of closed $L \otimes R$-invariant submodules $\left\{\mathcal{M}_{\Delta}\right\}_{\Delta \subset X}$ defined as follows. Fix a neighborhood $\Delta$ of the subgroup $Z$ in $X$ and consider a neighborhood $\Delta_{a}=\omega_{a}+\Delta$ of an orbit $\omega_{a} \subset X$. We put $I_{\Delta}(a)=I\left(\Delta_{a}\right)$ to be the minimal closed ideal of $B$ with the hull $\Delta_{a}$. Obviously, $R_{u} I_{\Delta}(a)=I_{\Delta}\left(a^{u}\right)(a \in \Omega, u \in U)$. Define a closed submodule $\mathcal{M}_{\Delta}$ similarly to the definition (5.6.3) of $\mathcal{M}_{0}$,

$$
\mathcal{M}_{\Delta}=\left\{f \in \Gamma^{m}(\Omega, \mathcal{E} \otimes B) \mid f(a) \in E(a) \otimes I_{\Delta}(a) \forall a \in \Omega\right\} .
$$

Obviously, $\mathcal{M}_{\Delta}$ is a closed $L \otimes R$ invariant subspace of $A=\Gamma^{m}(\Omega ; \mathcal{E} \otimes B)$ and the union $\bigcup_{\Delta \supset Z} \mathcal{M}_{\Delta}=\mathcal{M}_{0}$. This implies (IV). 
Notice that until now we have not used any specific property of the Fourier algebra $B=\mathcal{A}_{\rho}(X)$. So (I), (III) and (IV) hold for any commutative regular and Wiener algebra $B$ and a representation $R$ of $U$ on $B$.

The proof of (II) however will require some specific properties of $A_{\rho}(X)$ and the above representation $R$.

We recall that $U$ acts on $\mathcal{A}_{\rho}(X)$ by translations,

$$
\left(R_{u} f\right)(x)=f(x+l(u)) \quad\left(f \in \mathcal{A}_{\rho}(X) \forall u \in U\right),
$$

where $l: U \rightarrow X$ is a homomorphism. So in fact a commutative factor group $Y=l(U)$ acts on $B$. The infinitesimal operators of this representation $R$ are partial derivatives "along directions $Y$ " lifted to the Lie algebra $\mathfrak{H}$ of $U$. Precisely, we choose a system of coordinates $\left(y_{1}, \ldots, y_{r}\right)$ in the connected component $Y_{0}$ of $Y, Y_{0} \cong \mathbf{T}^{r}(r=\operatorname{dim} Y)$ and set $\partial_{y}^{\nu}$ for a partial derivative $\partial^{|\nu|} / \partial_{y_{1}}^{\nu_{1}} \cdots \partial_{y_{r}}^{\nu_{r}}$ on $X$. Smooth vectors of the representation $R$ are functions $f \in A_{\rho}(X)$ which are $m$-times differentiable in variables $y \in Y_{0}$ and such that $\partial_{y}^{\nu} f \in A_{\rho}(X) \forall|\nu| \leq m$. The $m$-norm of $f$ is given by

$$
\|f\|_{m}=\sum_{|\nu| \leq m}\left\|\partial_{y}^{\nu} f\right\|_{\mathcal{A}_{\rho}(X)},
$$

Let us notice that the $m$-norm (5.7.1) is equivalent to a weighted $L_{\rho^{\prime}}^{1}$-norm on the dual (discrete) group $A=\hat{X}$, i.e., $B_{m}$ is equal to another Fourier algebra $A_{\rho^{\prime}}(X)$. The weight $\rho^{\prime}$ on $A$ is described as follows. We consider the annihilator subgroup $B=Y_{0}^{\perp} \subset A$ and the factor group $A / B \cong \mathbf{Z}^{r}$. For an element $z=\left(z_{1}, \ldots, z_{r}\right) \in \mathbf{Z}^{r}$ we set

$$
\rho_{m}(z)=1+\sum_{|\nu| \leq m}\left|z^{\nu}\right| \sim 1+|z|^{m},
$$

where $|z|=\left|z_{1}\right|+\cdots+\left|z_{r}\right|$.

The weight function $\rho_{m}$ "pulls back" from the factor group $\mathbf{Z}^{r}=A / B$ to the whole group $A$. We denote by $\rho^{\prime}$ the product $\rho \rho_{m}$. Then $m$-norm (5.7.1) is equivalent to $L_{\rho^{\prime}}^{1}$-norm.

Now let $X$ be a compact abelian Lie group, $Y \subset X$ a connected subgroup of $X$ and $Z \subset Y$ a closed subgroup of $Y$. We consider the Fourier algebra $B=A_{\rho}(X)$ with a finite weight $\rho$ and a dense subalgebra $B_{m} \cong A_{\rho^{\prime}}(X)\left(\rho^{\prime}=\rho \rho_{m}\right)$.

Let us define the following two ideals of $B_{m}: I^{\prime}=I^{\prime}(Z)$ the minimal closed ideal of $B_{m}=A_{\rho^{\prime}}(X)$ with hull $Z$ and

$$
\tilde{I}=\tilde{I}(Z)=\left\{f \in B_{m}\left|\partial_{y}^{\nu} f \in I(Z) \forall\right| \nu \mid \leq m\right\},
$$

where $I(Z)$ denotes the minimal closed ideal of the algebra $B=A_{\rho}(X)$ with hull $Z$. They correspond to subspaces $\overline{I_{0}(a) \cap B_{m}}$ (the closure in $B_{m}$ ) and $I(a)_{m}$ in notations of (III).

We have to prove the equality $I^{\prime}=\tilde{I}$. This is done by reducing the problem to the case of the trivial group $Z=\{0\}$, i.e., primary ideals $I^{\prime}$ and $I$. The argument is similar to Theorem 4 . We observe that both ideals are invariant under the "regular representation" of the subgroup $Z$ on $X$, and hence decompose into the sum of $\chi$-primary components $I^{\prime}(\chi) \subseteq \tilde{I}(\chi)(\chi \in \hat{Z})$.

Then we show that the equality $I^{\prime}(\chi)=\tilde{I}(\chi)$ for any $\chi$ is equivalent to the equality of the "trivial" components $I^{\prime}(\mathbf{1})=\tilde{I}(\mathbf{1})(\mathbf{1}$ is a trivial character on $Z)$. 
The transition from 1- to $\chi$-components is accomplished by multiplication with a smooth nonvanishing function $\phi \in C^{\infty}(X)$, which "transforms according to $\chi$ ",

$$
\phi(x+z)=\chi(z) \phi(x) \quad \forall x \in X, z \in Z .
$$

Obviously any such $\phi$ maps $B(\mathbf{1})$ onto $B(\chi)$ and preserves minimal nonclosed ideals. So $\phi I^{\prime}(\mathbf{1})=I^{\prime}(\chi)$ in the algebra $B_{m}=A_{\rho^{\prime}}(X)$.

To show the equality $\phi \tilde{I}(\mathbf{1})=\tilde{I}(\chi)$ for the ideal $\tilde{I}$ we use the Leibniz formula

$$
\partial_{y}^{\nu}(\phi f)=\sum_{\mu}\left(\begin{array}{l}
\nu \\
\mu
\end{array}\right) \partial_{y}^{\nu-\mu}(\phi) \partial_{y}^{\mu}(f) \subset I=I(Z) \subset B,
$$

which implies $\phi \tilde{I}(\mathbf{1}) \subset \tilde{I}(\chi)$. But the inverse function $1 / \phi \in C^{\infty}(X)$ takes $\tilde{I}(\chi)$ back into $\tilde{I}(\mathbf{1})$. So both are equal.

This reduces the problem to verification of the equality $I^{\prime}(\mathbf{1})=\tilde{I}(\mathbf{1})$. But the 1 components $I^{\prime}(\mathbf{1})$ and $\tilde{I}(\mathbf{1})$ are naturally identified with primary ideals at the point $\{0\}$ of the algebra $A_{\rho^{\prime}}\left(X_{1}\right)=A_{\rho \rho_{m}}\left(X_{1}\right)$ on the factor-group $X_{1}=X / Z$. Namely, $I^{\prime}(1)$ is the minimal primary ideal at $\{0\}$ of $A_{\rho^{\prime}}\left(X_{1}\right)$, while $\tilde{I}(\mathbf{1})$ is characterized similar to (5.7.2).

Thus we arrive at the following situation: a compact abelian Lie group $X$ with a closed connected subgroup $Y \subset X, Y \cong \mathbf{T}^{r}$, acting by translations $R_{y}$ on $X$, a pair of Banach algebras $B=A_{\rho}(X)$ and $B_{m}=A_{\rho \rho_{m}}(X)(m$-smooth vectors of $B$ with respect to $R)$, and a pair of primary ideals of $B_{m}: I^{\prime}=I^{\prime}(0)$ the minimal primary ideal at $\{0\}$ and the ideal

$$
\tilde{I}=\left\{f \in B_{m} \mid\left(\partial_{y}^{\nu} f\right) \in I(0) \text { - the minimal primary ideal of } B \text { at }\{0\} \forall|\nu| \leq m\right\} \text {. }
$$

We want to show that $I^{\prime}=\tilde{I}$. For this we shall use the following well-known characterization of minimal primary ideals of Fourier algebras $A_{\rho}(X)$ with a finite weight $\rho[6, \S 38]$.

Let $I=I(0)$ be a minimal primary ideal of $\mathcal{A}_{\rho}(X)$ at $\{0\}$. The annihilator of $I$ consists of all pseudomeasures $\psi=\sum c_{\nu} \partial^{\nu}\left(\delta_{0}\right)\left(\delta_{0}\right.$ is a Dirac measure at $\left.\{0\}\right)$ such that the inverse Fourier transform of $\partial^{\nu} \delta_{0},\left(\partial^{\nu} \delta_{0}\right)^{\wedge}(z)=(i z)^{\nu}$, belongs to the space $L_{\rho}^{\infty}(A)$, i.e., $\sup \left\{\left|z^{\nu}\right| / \rho(z) \mid z \in \mathbf{Z}^{k}\right\}<\infty$.

We decompose the dual group $A=\mathbf{Z}^{r} \oplus \mathbf{Z}^{k-r} \oplus F$, where $\left(\mathbf{Z}^{k-r} \oplus F\right)=Y^{\perp}, \mathbf{Z}^{r} \cong$ $\hat{Y}$ and $F$ is finite. By definition (5.7.3) the annihilator of the ideal $\tilde{I} \subset L_{\rho \rho_{m}}^{1}(A)$ consists of all monomials $\left\{z^{\nu+\mu}\right\}, \nu=\left(\nu_{1}, \ldots, \nu_{k}\right), \mu=\left(\mu_{1}, \ldots, \mu_{r}\right)$ with $z^{\nu} \in$ $L_{\rho}^{\infty}(A)$ and $|\mu| \leq m$. But this is precisely the set of monomials which annihilate the minimal primary ideal $I^{\prime} \subset L_{\rho \rho_{m}}^{1}(A)$. Therefore, $I^{\prime}=\tilde{I}$. Q.E.D.

Lemma 3 of $\S 3$ follows from the results of this section.

Indeed, by Theorem 3 and Lemma 6 the equality $I(\omega)=\tilde{I}(\omega)$ in the space $C^{m}\left(\mathbf{R}^{n} ; B\right)$ is equivalent to equalities $\mathcal{M}_{l}=\overline{\mathcal{M}}_{0, l}(0 \leq l \leq m)$ in the spaces of sections $\Gamma^{m-l}\left(\Omega ; S_{l}(\mathcal{N}) \otimes B\right)$, while Theorem 4 and Lemma 7 establish the equalities $\mathcal{M}_{l}=\bar{M}_{0, l}$ under assumptions of Lemma 3 .

REMARK 2. Let us note that Lemma 3 holds for any smooth action of $U$ on $\mathbf{R}^{n}$ (not necessarily affine, as formulated in $\S 3$ ). The only modification which is required is a new definition of the "normal" bundle $(\mathcal{N}, \Omega)$. Since there is no inner product on $\mathbf{R}^{n}$ which is preserved by differentials $d u$ of smooth maps $u: \mathbf{R}^{n} \rightarrow$ 
$\mathbf{R}^{n}(u \in U)$, we do not have an invariant orthogonal complement $N_{a}$ of a tangent space $T_{a}=T_{a}(\Omega) \subset \mathbf{R}^{n}(a \in \Omega)$. However (see Example 3 of the Introduction), a representation $\tau(a, \cdot)$ of a stabilizer $U_{a}$ is defined on $\mathbf{R}^{n}$ and the tangent space $T_{a}$ is invariant under $\tau(a, \cdot)$. By Weyl's "complete reducibility" principle it possesses an invariant complement $T_{a}^{\prime}$ in $\mathbf{R}^{n}$. One easily verifies that for any $u \in U$, the differential $d u$ maps $T_{a}^{\prime}$ onto $T_{a^{u}}^{\prime}$. In particular the union $\tau^{\prime}=\bigcup_{a \in \Omega} T_{a}^{\prime}$ and consequently all symmetric-tensor powers $S_{k}\left(\tau^{\prime}\right)=\bigcup_{a \in \Omega} S_{k}\left(T_{a}^{\prime}\right)$ have a natural structure of smooth $U$-homogeneous vector bundles.

REMARK 3. A version of Whitney's Theorem proved in $\S 5$ can be posed in a more abstract setting of Banach algebras. Let $B$ be a Banach algebra upon which a Lie group $G$ acts by automorphisms. As above (5.4) we denote by $R$ the representation of $G$ on $B$ and by $R^{\nu}\left(\nu=\left(\nu_{1}, \ldots, \nu_{k}\right), k=\operatorname{dim} G\right)$ its infinitesimal operators. Then all subspaces of $m$-smooth vectors $B_{m}(m=0,1,2, \ldots)$ are also Banach algebras.

We take a nonclosed ideal $I_{0} \subset B$, whose $B$-closure is denoted by $I$, and define

$$
\tilde{I}=\left\{f \in B_{m}\left|R^{\nu} f \in I \forall\right| \nu \mid \leq m\right\} .
$$

One can show that $\tilde{I}$ is a closed (in $m$-norm) ideal of $B_{m}$. An abstract form of Whitney's Theorem is the following statement: the closure of $I_{0} \cap B_{m}$ in $B_{m}$ is equal to $\tilde{I}$.

In $\S 5$ we have proved this conjecture for the Fourier algebra $B=A_{\rho}(X)$ on a compact abelian Lie group $X$, when a subgroup $Y \subset X$ acts on $X$ by translations and $I_{0}$ is a minimal (nonclosed) ideal of $B$ with hull $Z \subset Y$ (Lemma 7).

We have also shown how the problem for the algebra $C_{0}^{m}\left(\mathbf{R}^{n}, B\right)$ reduces to that for $B$. Indeed, condition (II) of Theorem 4 is exactly "Whitney's property" of $B$.

It would be interesting to find other examples, which confirm or disprove this conjecture.

\section{Applications of Theorem 1.}

6.1. Burnside type theorem for Banach representations of motion groups. Let $G=A \rtimes U$ be a motion group, i.e., a semidirect product of LCA group $A$ and a compact group $U$ acting by automorphisms on $A$.

An immediate consequence of Theorem 1 and [10, Theorem 2], is the following version of the Burnside type theorem for motion groups. Let us call a Banach representation $g \rightarrow T_{g}$ of the motion group $G$ subpolynomial if the weight $\rho(a)=$ $\left\|T_{a}\right\|(a \in A)$ is subpolynomial.

THEOREM 5. If $T$ is a subpolynomial topologically irreducible representation of a motion group $G$, then $T$ is completely irreducible and therefore [9, Theorem 1] is Naimark-equivalent to a unitary irreducible representation of $G$.

Indeed, the group algebra $L_{\rho}^{1}(G)$ has a crossed product structure $\mathcal{L}=\mathcal{L}(U ; A)$ with a commutative regular and Wiener algebra $A=L_{\rho}^{1}(A)$ and a compact group $U$ acting by automorphisms on $A$,

$$
u: f \rightarrow f\left(a^{u}\right) \quad(f \in \mathcal{A}, u \in U) .
$$

By Theorem 1 all representations $R^{\omega}(\omega \subset \hat{A})$ are FM. Then by [10, Theorem 2 ] any topologically irreducible subpolynomial representation $T$ of $G$ is completely 
irreducible and since $A$ is also symmetric $T$ is Naimark-equivalent to a unitary irreducible representation of $G[\mathbf{9}$, Theorem 1].

As in $[14,10]$, Theorem 5 implies that in the class of subpolynomial representations of motion groups (respectively subpolynomial weights on $G$ ) all notions of irreducibility are essentially equivalent and therefore all different dual objects of $\mathcal{L}=L_{\rho}^{1}(G)$ coincide.

COROLLARY 3. Let $\rho$ be a subpolynomial weight on a motion group $G, \mathcal{L}=$ $L_{\rho}^{1}(G)$. The following dual objects of $\mathcal{L}$ are identical:

$$
\operatorname{Prim}_{t} \mathcal{L}=\operatorname{Prim}_{c} \mathcal{L}=\operatorname{Prim}_{a} \mathcal{L}=\operatorname{Prim}_{*} \mathcal{L}=\hat{G}_{*}=\operatorname{Max} \mathcal{L} .
$$

Here Prim with a subscript denotes the space of all primitive ideals (i.e., kernels of irreducible representations) for topological, complete, algebraic and unitary irreducibility, $\hat{G}_{*}$ is the unitary dual object of $G$ and $\operatorname{Max} \mathcal{L}$ the space of all maximal two sided ideals of $\mathcal{L}$.

REMARK 4. In special cases the restrictions on the repesentation's growth can be dropped. We shall mention one such class of groups: $G=A \rtimes U$ with almost compact abelian group $A$ (i.e., $A$ which coincides with the union of its compact subgroups). Natural examples are nonarchimedean motion groups $G=\mathbf{F}^{n} \rtimes$ $\mathrm{SL}(n, \mathbf{Z})$, where $\mathbf{F}$ is a nonarchimedean field, $\mathbf{Z}$ is the ring of integers of $\mathbf{F}$.

For such groups Theorem 1 and therefore Theorem 5 and Corollary 3 hold for any weight $\rho$ on $G$, respectively for all Banach representations of $G$.

Indeed, the vector component $\mathbf{R}^{n}=0$ in this case and any weight on $A=D$ is uniformly finite with $m(\rho)=1$.

REMARK 5. Topologically irreducible representations naturally appear in the study of one-sided ideals of Banach algebras. Indeed, every maximal closed left (or right) ideal $\mathcal{N}$ of $\mathcal{L}$ defines a topologically irreducible representation $R$ of $\mathcal{L}$ on the quotient space $\mathcal{L} / \mathcal{N}$. The Burnside type theorem reduces the problem of characterization of all maximal left ideals from $\mathcal{L}$ to its ${ }^{*}$-primitive quotients $\mathcal{L} / \mathcal{M}$. For the latter the problem was solved in [14]. So Theorem 5 combined with [14] yields a complete characterization of maximal left ideals of the group algebra $L_{\rho}^{1}(G)$ on a motion group $G$ with a subpolynomial weight $\rho$.

6.2 Primary ideals of invariant subalgebras. Let a compact group $U$ act by automorphisms on the Banach algebra $A$. We consider the subalgebra $Z=A^{U}$ of all $U$-stable elements of $A$, i.e.,

$$
Z=\left\{f \in \mathcal{A} \mid f^{u}=f \forall u \in U\right\} .
$$

If $A=L_{\rho}^{1}(A)$ and $U$ acts by automorphisms on $A$, then $Z=L_{\rho}^{1}(A)^{U}$ consists of all $U$-invariant functions on $A$.

These algebras retain some basic properties of $\mathcal{A}$, e.g., the Wiener property, regularity, symmetry, etc., but usually they violate Ditkin's property (cf. [24]). It is still possible however that a weaker version of Ditkin's property, the property (F) of the introduction, holds.

Theorem 1 yields a positive answer to this problem for group algebras $L_{\rho}^{1}(A)$, equivalently Fourier algebras $A_{\rho}(X)$.

Indeed, since a quotient algebra $Z / I(\omega)$ ( $Z$ modulo the minimal primary ideal of $Z$ with the hull $\{\omega\})$ is identified with a subspace of $U$-invariants in $A / I(\omega)$, 
property (R) implies

$$
\operatorname{dim} Z / I(\omega)=k\left(\mathbf{1} ; R^{\omega}\right)<\infty .
$$

Thus we get the following generalization of Varopouloses' result [24].

COROLlaRY 4. Let $A$ be a LCA group and let a compact group $U$ act by automorphisms of $A$. For any subpolynomial weight $\rho$ on $A$ the subalgebra $Z=$ $\mathcal{Z}^{U} L_{\rho}^{1}(A)$ of the group algebra $L_{\rho}^{1}(A)$ satisfies property $(\mathrm{F})$. Moreover, codimensions of minimal primary ideals of $Z$ are bounded by the constant $c(n, \rho)$ of Theorem 1.

More general invariant subalgebras appear in the context of so-called $[F C]_{B^{-}}^{-}$ groups. We recall their definition (for more details, see $[8,5,18]$ ). A pair of a LC group $G$ and a subgroup $B$ of its automorphisms, which contains all inner automorphisms $I(G)$, is called a $[F C]_{B}^{-}$-group if all $B$-orbits $B[x]=\left\{x^{u} \mid u \in B\right\}$ are relatively compact in $G$.

Of course all pairs $(A, U)$ of a LCA group $A$ and a compact group $U$ acting by automorphisms of $A$ belong to this class.

In the case of $B=I(G)$ we get so-called $[F C]^{-}$-groups, i.e., groups with relatively compact conjugacy classes.

The structure of their group algebras $L_{\rho}^{1}(G)$ and harmonic analysis on them were studied in several papers (see, for instance, $[\mathbf{8}, \mathbf{1 5}, \mathbf{1 8}]$ ). The following two subalgebras of $L_{\rho}^{1}(G)$ proved to be important in this study: $Z L_{\rho}^{1}(G)$ the centre of $L_{\rho}^{1}(G)$ and a $B$-fixed subalgebra

$$
Z^{B} L_{\rho}^{1}(G)=\left\{f \in L_{\rho}^{1}(G) \mid f\left(x^{u}\right)=f(x) ; x \in G, u \in B\right\} .
$$

Obviously, $Z L_{\rho}^{1}(G)=Z^{I(G)} L_{\rho}^{1}(G) \supseteq Z^{B} L_{\rho}^{1}(G)$. These subalgebras are always commutative semisimple Banach algebras. In [15] a sufficient condition on the weight $\rho$ was given in order that $Z^{B} L_{\rho}^{1}(G)$ is regular and Wiener. In [18] Ditkin's condition and property $(\mathrm{F})$ were studied. In particular, property $(\mathrm{F})$ was proved for any $B$-invariant subalgebra $Z^{B} L_{\rho}^{1}(G)$ of a Beurling group algebra $L_{\rho}^{1}(G)$ with a polynomial weight $\rho$, under the following two assumptions.

(1) Group $G$ is compactly generated. [8]).

(2) $B$ acts on $G$ by so-called "automorphisms of bounded displacement" (see

The second condition holds for all $[F C]^{-}$-groups $G$ i.e., when $B=I(B)$, but fails in general.

The results of $\S \S 2$ and 3 (Theorem 1 and Lemma 1) enable us to drop both these restrictions, i.e., to prove property (F) for any $[F C]_{B}^{-}$-group $G$ and a $B$-invariant subalgebra $Z^{B} L_{\rho}^{1}(G)$. Of course, the polynomial growth condition must be replaced by a "subpolynomial" condition similar to Definition 1 of $\S 1$.

To define a notion of a finite and uniformly finite weight for $[F C]^{-}$groups we need some structural results concerning this class (see [8]).

(S1) Any $[F C]_{B}^{-}$-group $G$ coincides with the union of its open compactly generated $B$-invariant subgroups $\left\{G_{\alpha}\right\}_{\alpha}$, i.e., $G=\lim _{\alpha}$ ind $G_{\alpha}$.

(S2) Any compactly generated $[F C]_{B}^{-}$-group $G$ contains a maximal compact characteristic subgroup $K$ such that $G / K$ is abelian (and even torsion free).

(S3) Vector components $\left\{L_{\alpha}\right\}_{\alpha}$ of groups $\left\{G_{\alpha} / K_{\alpha}\right\}_{\alpha}$ stabilize after finitely many steps. In particular, $n=\sup \left\{\operatorname{dim} L_{\alpha} \mid \alpha\right\}<\infty$. 
Now let $\rho$ be a weight on $G$. Restricted on each compactly generated subgroup $G_{\alpha}$ it is equivalent to a $K$-invariant weight

$$
\rho_{\alpha}(a x)=\rho_{\alpha}(a) \quad \forall a \in G_{\alpha}, x \in K_{\alpha} .
$$

Therefore $\rho_{\alpha}$ factors through the weight of the commutative factor-group

$$
A_{\alpha}=G_{\alpha} / K_{\alpha}, \rho_{\alpha}(\dot{a})=\rho_{\alpha}(a) \quad\left(a \in G_{\alpha}, \dot{a}=a K_{\alpha}\right) .
$$

Let us say that $\rho$ is uniformly finite if all $\left\{\rho_{\alpha}\right\}_{\alpha}$ are finite (cf. $§ 1$ ) and the degrees of finiteness of $\left\{\rho_{\alpha}\right\}_{\alpha}$ are uniformly bounded,

$$
\sup \left\{m\left(\rho_{\alpha}\right) \mid \alpha\right\}=m<\infty .
$$

If, furthermore, each $\rho_{\alpha}$ is polynomial on the vector component of $A_{\alpha}$ we shall call $\rho$ subpolynomial.

THEOREM 6. Let $G$ be $a[F C]_{B}^{-}$-group and $\rho$ be a subpolynomial weight on $G$. The $B$-fixed subalgebra $Z=Z^{B} L_{\rho}^{1}(G)$ of the Beurling group algebra $L_{\rho}^{1}(G)$ satisfies property $(\mathrm{F})$. Moreover $\operatorname{dim} Z / I(x) \leq c(n, \rho)$, the constant of Theorem 1 , where $n=\sup \left\{\operatorname{dim} L_{\alpha} \mid \alpha\right\}$.

Proof. We consider the centre $A=Z L_{\rho}^{1}(G)$ and the $B$-invariant subalgebra $Z=Z^{B} L_{\rho}^{1}(G)$. We also introduce algebras $A_{\alpha}=Z L_{\rho}^{1}\left(G_{\alpha}\right)$ and $Z_{\alpha}=Z^{B} L_{\rho}^{1}\left(G_{\alpha}\right)$, according to $(\mathrm{S} 1)$.

The embedding $p_{\alpha}: Z_{\alpha} \rightarrow Z$ defines a continuous map of Gelfand spaces, $p_{\alpha}^{*}: \hat{Z}$ $\rightarrow \hat{Z}_{\alpha}$. Let $x \in \hat{Z}$ and $x_{\alpha}=p_{\alpha}^{*}(x)$ be its image in $\hat{Z}_{\alpha}$. The homomorphisms $p_{\alpha}$ take the minimal primary ideal $I\left(x_{\alpha}\right) \subset Z_{\alpha}$ into the minimal primary ideal $I(x)$ of $Z$. So it factors through a homomorphism of quotient-algebras

$$
W_{\alpha}: Z_{\alpha} / I\left(x_{\alpha}\right) \rightarrow Z / I(x) .
$$

The images $\left\{W_{\alpha}\left(Z_{\alpha} / I\left(x_{\alpha}\right)\right)\right\}_{\alpha}$ form a partially ordered family of subspaces in $Z / I(x)$ and their linear span is dense in $Z / I(x)$. As in the proof of Theorem 1 it suffices to show that all quotients $\left\{Z_{\alpha} / I\left(x_{\alpha}\right)\right\}_{\alpha}$ are finite dimensional and the numbers $d_{\alpha}=\operatorname{dim}\left(Z_{\alpha} / I\left(x_{\alpha}\right)\right)$ are uniformly bounded,

$$
d_{\alpha} \leq d \quad \forall \alpha .
$$

Then the "inductive limit" argument of Proposition 3 yields $\operatorname{dim} Z / I(x) \leq d$.

So let us assume that $G$ is a compactly generated $[F C]_{B}^{-}$-group. As above we define the subalgebras $A=Z L_{\rho}^{1}(G)$ and $Z=Z^{B} L_{\rho}^{1}(G)$.

We shall use the following results of $[8,15]$ concerning the structure of $A=$ $Z L_{\rho}^{1}(g)$ :

(S4) The Gelfand space $X$ of $A$ decomposes into the union of open disjoint subsets $\left\{X_{\lambda}\right\}_{\lambda \in \Lambda}$. Each $X_{\lambda}$ is identified with the dual of an abelian factor-group $H_{\lambda} / K$, where $H_{\lambda}$ is an open normal subgroup of $G$, and $K$ is a compact characteristic subgroup of $G$ (cf. (S2)).

(S5) The ideal $A_{\lambda}=\left\{f \in A \mid \operatorname{supp} \hat{f} \subset X_{\lambda}\right\}$ is canonically isomorphic to the Beurling group algebra $L_{\rho}^{1}\left(H_{\lambda} / K\right)$.

(S6) The ideals $\left\{\mathcal{A}_{\lambda}\right\}_{\lambda \in \Lambda}$ span a dense subspace in $\mathcal{A}$. 
We denote by $U$ the closure of $B$ in the group Aut $G$, equipped with the compactopen topology. The group $U$ is known to be compact (see $[8]$ ), and it acts by automorphisms on $A$,

$$
u: f(x) \rightarrow f\left(x^{u}\right) \quad(f \in \mathcal{A}, u \in U) .
$$

The subalgebra $Z \subset A$ coincides with the set of all $U$-invariants of $\mathcal{A}$. Let us also notice that the compact group $U$ acts on the discrete space $\Lambda$, i.e., $X_{\lambda}^{u}=X_{\lambda^{u}} \forall \lambda \in$ $\Lambda, u \in U$ (cf. [18]). Let $\omega \subset X$ be an orbit of $U$ in $X$. We shall prove that the representation $R^{\omega}$ of $U$ on the quotient space $A / I(\omega)$ is FM and the multiplicities $k\left(\pi ; R^{\omega}\right)$ are estimated as in Theorem 1 .

According to (S4) $\omega$ splits into the finite union $\bigcup_{\lambda \in \omega} \omega_{\lambda}$ of disjoint subsets $\omega_{\lambda}=\omega \cap X_{\lambda}$, each of which is an orbit of a stabilizer $U_{\lambda}$. As in the proof of Lemma $3(\S 3.4)$ the representation $R^{\omega}$ in the quotient space $A / I(\omega)$ is induced by $R^{\omega_{\lambda}}$ acting on the space $A_{\lambda} / I\left(\omega_{\lambda}\right)$.

The stabilizer $U_{\lambda}$ acts by automorphisms on $H_{\lambda}$ and consequently on the abelian factor group $H_{\lambda} / K$. The action of $U_{\lambda}$ on $X_{\lambda}$ is dual to its action $H_{\lambda} / K$ and the representation $R^{\omega_{\lambda}}$ arises from this (automorphic) action. Let us also notice that by (S5) the representation space $A_{\alpha}$ is isomorphic to the group algebra $L_{\rho}^{1}\left(H_{\lambda} / K\right)$. Thus all the assumptions of Lemma $1(\S 3)$ are satisfied and we get

$$
k\left(\pi ; R^{\omega_{\lambda}}\right) \leq c(n, \rho) d(\pi) \quad \forall \pi \in \hat{U}_{\lambda} .
$$

Then Proposition 7 yields the same estimate for the induced representation,

$$
k\left(\pi ; R^{\omega}\right) \leq c(n, \rho) d(\pi) .
$$

In particular, for the trivial representation $1 \in \hat{U}$ we obtain

$$
\operatorname{dim} Z / I(\omega)=k\left(\mathbf{1} ; R^{\omega}\right) \leq c(n, \rho) .
$$

The right-hand part of $(6.2 .5), c(n, \rho)=m\left(\rho^{\prime \prime}\right) d\left(\tau_{m}\right)$ depends only on $\rho$ and the dimension of the vector component of $H_{\lambda} \subset G\left(\cong G_{\alpha}\right)$. Since dimensions of vector components $L_{\alpha}$ of LCA groups $G_{\alpha} / K_{\alpha}$ are bounded, $n_{\alpha}=\operatorname{dim} L_{\alpha} \leq n$, we get the required uniform estimates (6.2.2). The theorem is proved.

Appendix A. We shall prove Lemma 5 by induction in the degree of smoothness $m$. For $m=0, \Gamma(\Omega ; \mathcal{E} \otimes B)=D_{0}=D$, by definition.

Assume that $\Gamma^{m-1}(\Omega ; \mathcal{E} \otimes B)=D_{m-1}$.

In order to characterize the spaces $\Gamma^{m}(\Omega ; \mathcal{E} \otimes B)$ and $D_{m}$ we proceed to local coordinates of the smooth vector bundle $(\mathcal{E}, \Omega)$. In local coordinates each section $f \in \Gamma(\Omega ; \mathcal{E} \otimes B)$ turns into a vector-valued function $\tilde{f}: \mathbf{R}^{k} \rightarrow B \otimes E$, where $k=\operatorname{dim} \Omega$ and $E \cong E_{a}(a \in \Omega)$.

By definition, $f \in \Gamma^{m}(\Omega ; \mathcal{E} \otimes B)$ iff $\tilde{f}$ is $m$-smooth, i.e., $\tilde{f} \in C^{m}\left(\mathbf{R}^{k} ; B \otimes E\right)$, which is equivalent to

$$
\partial_{j} \tilde{f} \in C^{m-1}\left(\mathbf{R}^{k} ; B \otimes E\right)
$$

for all partial derivatives $\partial_{j}$ on $\mathbf{R}^{k}$. On the other hand $f \in D_{m}$ iff $L_{h}(f) \in$ $D_{m-1} \forall h \in \mathfrak{H}$, which is equivalent by the inductive assumption to

$$
\left(L_{h} f\right)^{\sim} \in C^{m-1}\left(\mathbf{R}^{k} ; B \otimes E\right) \quad \forall h \in \mathfrak{H} .
$$


In order to check the equivalence of (A.1) and (A.2) we rewrite a first order differential operator $L_{h}$ on $(\mathcal{E}, \Omega)$ in local coordinates. Owing to local triviality it has the form (5.3.8), i.e.

$$
\left(L_{h} f\right)^{\sim}(x)=\sum_{j=1}^{k} \alpha_{j}(h, x) \partial_{j} \tilde{f}+B(h, x) \tilde{f}(x),
$$

where $\alpha_{j}(h, \cdot)$ are scalar and $B(h, \cdot)$ is a $\operatorname{Hom}(E ; E)$-valued $C^{\infty}$-function.

Now let $f \in \Gamma^{m}(\Omega ; \mathcal{E} \otimes B)$, which by (A1) is equivalent to $\partial_{j}^{\sim} f \in C^{m-1}, j=$ $1, \ldots, k$. Then of course, $L_{h}^{\sim} f \in C^{m-1} \forall h \in \mathfrak{H}$ which by (A.2) implies $f \in D_{m}$. Thus we have proved that

$$
\Gamma^{m}(\Omega ; \mathcal{E} \otimes B) \subseteq D_{m}
$$

Conversely, let $f \in D_{m}$ which is equivalent to

$$
\left(L_{h}^{\sim} f\right)(x)=\sum_{j=1}^{k} \alpha_{j}(h, x) \partial_{j} \tilde{f}+B(h, x) \tilde{f}(x) \in C^{m-1} \quad \forall h \in \mathfrak{H}
$$

by the inductive assumption.

Notice that any $f \in D_{m}$ obviously belongs to $D_{m-1}$ and therefore $f(x)$ and $B(h, x) f(x)$ are $(m-1)$-smooth. Since the coefficient $B(h, x)$ is $\infty$-smooth we get

$$
\sum_{j=1}^{k} \alpha_{j}(h, x) \partial_{j} \tilde{f} \in C^{m-1} \quad \forall h \in \mathfrak{H}, f \in D_{m} .
$$

Let us choose elements $\left(h_{1}, \ldots, h_{k}\right)$ in $\mathfrak{H}$ such that the matrix $A(x)=$ $\left(\alpha_{j}\left(h_{i}, x\right)\right)_{i j=1}^{k}$ is invertible at a neighborhood of $0 \in \mathbf{R}^{k}$. Applying the inverse $C^{\infty}$-matrix function $A(x)^{-1}$ to the right-hand side of (5.4.11) we get $\partial_{j} \tilde{f} \in C^{m-1}$ $\forall j=1, \ldots, k$, which means that $f \in \Gamma^{m}(\Omega ; \mathcal{E} \otimes B)$. This proves the equality $\Gamma^{r}(\Omega ; \mathcal{E} \otimes B)=D_{m}$.

The above argument also shows the equivalence of both definitions of $m$-norm (5.4.2) for $\Gamma^{m}(\Omega ; \mathcal{E} \otimes B)$ and (5.4.3) for $D_{m}$.

Appendix B: Proof of Theorem 3. We want to show the isomorphism of spaces

$$
\mathbf{C}^{m}(\Omega ; B)=C^{m}\left(\mathbf{R}^{n} ; B\right) / I(\Omega ; B) \cong \bigoplus_{l=0}^{m} \Gamma^{m-l}\left(\Omega ; \varepsilon_{0, l} \otimes B\right) .
$$

To each Whitney function $F=\left(f_{\nu}\right)_{|\nu| \leq m}$ on $\Omega$ we attach a tuple of $r$-forms $\left(\phi^{(r)}\right)_{r=0}^{m} ; \phi^{(r)} \in \Gamma\left(\mathcal{E}^{(r)} \otimes B\right)$, whose matrix entries are coefficients $\left\{f_{\nu}\right\}$,

$$
F \rightarrow\left(\phi^{(r)}\right)_{r=0}^{m} \quad \text { s.t. } \phi^{(r)}(a ; \underbrace{\partial_{1}, \ldots, \partial_{1}}_{\nu_{1}} ; \underbrace{\partial_{2}, \ldots, \partial_{2}}_{\nu_{2}} ; \ldots)=f_{\nu}(a) .
$$

Each form $\phi^{(r)}$ in its turn splits into the sum of $(k, l)$-forms according to (5.2.9),

$$
\phi^{(r)}=\bigoplus_{k+l=r} \phi_{k, l} \text {. }
$$

We want to show that each $\phi^{(r)}$ is $(m-r)$-smooth, equivalently $\phi_{k, l}$ is $(m-k-l)$ smooth, and a tuple $\left(\phi^{(r)}\right)_{r=0}^{m}$ is uniquely determined by its normal components $\left(\phi_{0, l}\right)_{l=0}^{m}$. 
Conversely, any tuple $\left(\phi_{0, l}\right)_{l=0}^{m}$ as above gives rise to $\left(\phi^{(r)}\right)_{r=0}^{m}$ and consequently to a Whitney function $F=\left(f_{\nu}\right)_{|\nu| \leq m}$ by (B.2).

An essential step in the proof will be the following characterization of Whitney functions on a smooth manifold $\Omega$.

LEMMA 9. Let $\Omega \subset \mathbf{R}^{n}$ be a smooth submanifold.

(1) A tuple of continuous $B$-valued functions $\left(f_{\nu}\right)_{|\nu| \leq m}$ on $\Omega$ defines a Whitney function $F \in \mathbf{C}^{m}(\Omega ; B)$ iff:

(a) each $f_{\nu} \in C^{m-|\nu|}(\Omega ; B)$ (i.e., $f_{\nu}$ is $(m-|\nu|)$-smooth),

(b) for any tangent vector field $\xi \in \Gamma(\Omega ; \tau)$ the derivative of $f_{\nu}$ along $\xi=$ $\sum_{1}^{n} \alpha_{j} \partial_{j}$ is given by

$$
\partial_{\xi}\left(f_{\nu}\right)=\sum_{j=1}^{n} \alpha_{j} f_{\nu+\delta_{j}} \quad \forall|\nu| \leq m-1
$$

where $\delta_{1}, \ldots, \delta_{n}$ denote the basic multi-indices $\delta_{1}=(1,0, \ldots, 0) ; \delta_{2}=(0,1,0, \ldots)$, etc.

(2) The remainder $R^{\nu} F$ is estimated by the $L^{\infty}$-norm of the highest order terms $\left(f_{\mu}\right)_{|\mu|=m}$ of $F$, namely

$$
\sup \left\{\frac{\left(R^{\nu} F\right)(x, a)}{|x-a|^{m-|\nu|}} ; x, a \in Q\right\} \leq C \sum_{|\mu|=m}\left\|f_{\mu}\right\|_{L^{\infty}(Q)}
$$

with some constant $C>0$, depending on $\Omega$.

In particular, the norm (5.4.5) on $\mathbf{C}^{m}(\Omega ; B)$ is equivalent to the usual m-norm $\|F\|_{m}^{\prime} \sim\|F\|_{m}=\sum_{|\nu| \leq m}\left\|f_{\nu}\right\|_{L^{\infty}}$.

PROOF. (1) Necessity of conditions (a) and (b) easily follows from the definition (5.4.4) of Whitney functions after proceeding to local coordinates on $\Omega$.

Let us prove sufficiency. Notice that (5.4.4) is nothing but a "curved" version of the Taylor expansion of a smooth function $f$ (if $\Omega$ were "plane" Taylor's theorem would immediately imply the result). We shall modify the argument in Taylor's theorem in such a way that geodesics on $\Omega$ will play the role of straight lines in the Euclidean case.

Let us introduce the following notations:

(1) Let $j=\left(j_{1}, \ldots, j_{r}\right)$ be a tuple of integers $1 \leq j_{k} \leq n(k=1, \ldots, r)$.

(2) Let $\nu=\nu(j)=\left(\nu_{1} \cdots \nu_{n}\right)$ be a multiindex of $j$, i.e., $\nu_{1}$ is the number of 1 's among the integers $\left(j_{1}, \ldots, j_{r}\right) ; \nu_{2}$ is the number of 2's, ., etc. $\left(|\nu|=\nu_{1}+\nu_{2}+\right.$ $\left.\cdots+\nu_{n}=r\right)$.

(3) $\Delta^{(r)}$ will denote a simplex (" $r$-interval") $\Delta^{(r)}=\left\{\left(t_{1}, \ldots, t_{r}\right): 1 \geq t_{1} \geq t_{2} \geq\right.$ $\left.\cdots \geq t_{r} \geq 0\right\}$ and $Q^{(r)}$ will denote the $r$-cube $[0,1]^{r}$.

(4) If $\gamma=\left(\gamma_{1}, \ldots, \gamma_{n}\right)$ is a family of functions of bounded variation on $[0,1]$, then $d \gamma^{(j)}(t)$ will denote the measure $d \gamma_{j_{1}}\left(t_{1}\right) \cdots d \gamma_{j_{r}}\left(t_{r}\right)$ on $Q^{(r)}$.

Take a pair of points $a, x \in \Omega$ and a geodesic $\gamma:[0,1]_{n} \rightarrow \Omega$ with endpoints $\gamma(0)=a ; \gamma(1)=x$. Denote by $\left\{\gamma_{i}(t)\right\}_{i=1}^{n}$ the Euclidean coordinates of $\gamma(t)$. We shall use the same procedure as in the proof of Taylor's theorem, i.e. write the difference $f(x)-f(a)$ as a line integral $\int_{0}^{1}(d / d t)(f \circ \gamma(t)) d t$. But instead of straight line intervals, as in the classical case we shall use curves $\gamma(t)$. After the first step 
of the procedure we get

$$
f(x)=f(a)+\int_{0}^{1} \frac{d}{d t}(f \circ \gamma)(t) d t=f(a)+\sum_{j=1}^{n} \int_{0}^{1}\left(\partial_{j} f\right) \circ \gamma(t) d \gamma_{j}(t) .
$$

Writing each partial derivative $\left(\partial_{j} f\right) \circ \gamma(t)$ as

$$
\left(\partial_{j} f\right)(a)+\left[\left(\partial_{j} f\right) \circ \gamma(t)-\left(\partial_{j} f\right)(a)\right]
$$

and applying the same procedure to the difference $\left(\partial_{j} f\right) \circ \gamma(t)-\left(\partial_{j} f\right)(a)$ yields $f(x)=f(a)+\sum_{j}\left(\partial_{j} f\right)(a) \int_{0}^{1} d \gamma_{j}(t)+\sum_{j_{1} j_{2}} \int_{0}^{1} \int_{0}^{t_{1}}\left(\partial_{j_{1} j_{2}}^{2} f\right)\left(\gamma\left(t_{2}\right)\right) d \gamma_{j_{2}}\left(t_{2}\right) d \gamma_{j_{1}}\left(t_{1}\right)$.

After $r$ iterations we get an expansion

$$
\begin{aligned}
f(x)= & f(a)+\sum_{j}\left(\partial_{j} f\right)(a) \int_{0}^{1} d \gamma_{j}(t)+\cdots \\
& +\sum_{j=\left(j_{1} \ldots j_{r}\right)}\left(\partial_{j}^{r} f\right)(a) \int_{\Delta(r)} d \gamma^{(j)}(t)+R_{r}(a, x)
\end{aligned}
$$

with the remainder

$$
R_{r}(x, a)=\sum_{j} \int_{\Delta^{(r)}}\left[\left(\partial_{j}^{r} f\right) \circ \gamma\left(t_{r}\right)-\left(\partial_{j}^{r} f\right)(a)\right] d \gamma^{(r)}(t) .
$$

In the $r$ th sum of the expansion (B.3) we collect all tuples $j$, which correspond to the same multiindex $\nu$, i.e. $\nu(j)=\nu$, and write it as

$$
\sum_{|\nu|=r}\left(\partial^{\nu} f\right)(a) \sum_{j: \nu(j)=\nu} \int_{\Delta(r)} d \gamma^{(j)}(t)
$$

Next we use the following modification of the well-known "simplex volume identity"

$$
\sum_{j: \nu(j)=\nu} \int_{\Delta^{(r)}} d \gamma^{(j)}(t)=\frac{1}{\nu !}[\gamma(1)-\gamma(0)]^{\nu}=\frac{1}{\nu !}(x-a)^{\nu} .
$$

In the classical case $d \gamma_{i}=\left(x_{i}-a_{i}\right) d t(1 \leq i \leq n)$ and (B.5) amounts to evaluating the volume of $\Delta^{(r)}$.

To show (B.5) in the general case we take any product measure $d \mu(t)=$ $\prod_{i=1}^{r} d \mu_{i}\left(t_{i}\right)$ on $[0,1]^{r}$. For a permutation $\sigma \in \Sigma_{r}$ denote by $d \mu^{\sigma}$ the measure $\prod_{i=1}^{r=1} d \mu_{\sigma(i)}\left(t_{i}\right)$. Then

$$
\sum_{\sigma} \int_{\Delta^{(r)}} d \mu^{\sigma}(t)=\int_{Q^{(r)}} d \mu(t)=\prod_{i=1}^{r}\left(\mu_{i}(1)-\mu_{i}(0)\right) .
$$

Let us apply (B.6) to the measure $d \mu=d \gamma^{(j)}$. Since some factors of $d \mu$ are identical now, certain permutations $\sigma$ will result in the same measure $d \mu$. Precisely, we divide the interval $[1,2, \ldots, r]$ into $n$ disjoint subsets of the length $\nu_{1}, \nu_{2}, \ldots, \nu_{n}$ and denote by $\Sigma(\nu)$ the stabilizer of this partition. Then each term in the sum is repeated exactly $\nu !=\#\{\Sigma(\nu)\}$-times. Moreover there is 1-1 correspondence 
between all tuples $j=\left(j_{1} \cdots j_{r}\right)$ s.t. $\nu(j)=\nu$ and all cosets of $\Sigma_{r}$ modulo $\Sigma(\nu)$. The left-hand side of (B.6) thus becomes $\nu ! \sum_{j: \nu(j)=\nu} \int_{\Delta(r)} d \gamma^{(j)}$, while the righthand side is obviously $\prod_{i=1}^{r}\left(\gamma_{j_{1}}(1)-\gamma_{j_{i}}(0)\right)=\prod_{i=1}^{n}\left(\gamma_{i}(1)-\gamma_{i}(0)\right)^{\nu_{i}}$, whence the identity (B.5) follows.

The above procedure gives another derivation of the Taylor expansion of a smooth function $f$ on $\mathbf{R}^{n}$ and would be of little interest as such. But now we shall apply it to the tuple of functions $F=\left(f_{\nu}\right)_{|\nu| \leq m}$, satisfying assumptions (a) and (b) of Lemma 9. Notice that $f_{\nu}$ are defined only on the submanifold $\Omega \subset \mathbf{R}^{n}$, and they are not assumed smooth.

Take $f_{\nu}(|\nu| \leq m-1)$ and write the line integral along $\gamma$ for the difference $f_{\nu}(x)-f_{\nu}(a)$. By assumption (b) of the lemma

$$
f_{\nu}(x)-f_{\nu}(a)=\int_{0}^{1} \sum_{1}^{n} f_{\nu+\delta_{j}} \circ \gamma(t) d \gamma_{j}(t) .
$$

Here $f_{\nu+\delta_{j}}$ play the role of partial derivatives $\partial_{j} f_{\nu}$, which no longer exist.

Writing $f_{\nu+\delta_{j}} \circ \gamma(t)=f_{\nu+\delta_{j}}(a)+\left[f_{\nu+\delta_{j}} \circ \gamma(t)-f_{\nu+\delta_{j}}(a)\right]$, using (B.5) and repeating the above procedure $r=m-|\nu|$ times we get the following "Taylor series expansion" of $f_{\nu}$ :

$$
f_{\nu}(x)=\sum_{|\nu| \leq r} \frac{1}{\mu !} f_{\nu+\mu}(a)(x-a)^{\mu}+\left(R^{\nu} F\right)(x, a),
$$

with the remainder $R^{\nu} F$ given by (B.4), i.e.

$$
\left(R^{\nu} F\right)(x, a)=\sum_{j=\left(j_{1} \ldots j_{r}\right)} \int_{\Delta^{(r)}}\left[f_{\nu+\nu(j)} \circ \gamma\left(t_{r}\right)-f_{\nu+\nu(j)}(a)\right] d \gamma^{(j)}(t) .
$$

In order to estimate each summand in the right-hand side of (B.8) we choose a small neighborhood $Q$ of $\{a\}$ in $\Omega$ such that the Euclidean distance $|\gamma(t)-a|(0 \leq$ $t \leq 1)$ increases monotonically as $t$ grows, for any geodesic $\gamma:[0,1] \rightarrow Q$, with endpoints $\gamma(0)=a ; \gamma(1)=x$.

Notice that there exists a constant $c=c(Q)$, which dominates total variations of all $d \gamma_{i}(t)(0 \leq t \leq 1)$, i.e.,

$$
d\left|\gamma_{i}\right|(t) \leq c\left|x_{i}-a_{i}\right| d t, \quad i=1, \ldots, n .
$$

The constant $c$ depends on the curvature of $\Omega$ at $Q$.

We get the following estimate of the remainder (B.8):

$$
\begin{aligned}
& \mid \int_{\Delta^{(r)}} {\left[\left(f_{\nu+\nu(j)} \circ \gamma\right)\left(t_{r}\right)-f_{\nu+\nu(j)}(a)\right] d \gamma^{(j)}(t) \mid } \\
& \leq \max \left\{\left|f_{\nu+\nu(j)}(y)-f_{\nu+\nu(j)}(a)\right|: y \in \Omega \text { s.t. }|y-a| \leq|x-a|\right\} \\
& \quad \times \int_{\Delta^{(r)}} d\left|\gamma^{(j)}\right|(t)=O(1) c^{r}|x-a|^{r} / r ! \quad \text { as }|x-a| \rightarrow 0 .
\end{aligned}
$$

Obviously, this implies for all $\nu$ that

$$
\left(R^{\nu} F\right)(x, a)=o\left(|x-a|^{m-|\nu|}\right),
$$

i.e. the tuple $F=\left(f_{\nu}\right)_{|\nu| \leq m}$ is a Whitney function. 
We have proved the first statement of the lemma. To prove the second notice that (B.9) gives an estimate of the "extra term" of $\|\cdot\|_{m}^{\prime}$-norm (5.4.5) in terms of "highest order derivatives" $\left\{f_{\mu}\right\}_{|\mu|=m}$ of the tuple $F$. Indeed, for any compact $Q \subset \Omega$ there exists a constant $c=c(Q)$ such that (B.9) holds for all $a, x \in Q$. Then

$$
\begin{aligned}
\sup \left\{\frac{\left|R_{\nu} F(x, a)\right|}{|x-a|^{m-|\nu|}}: \forall x, a \in Q\right\} \leq c^{r} \frac{n^{r}}{r !} 2 \max \left\{\left\|f_{\mu}\right\|_{L^{\infty}(Q)}:|\mu|=m\right\} & \\
& (r=m-|\nu|) .
\end{aligned}
$$

Of course, $\|F\|_{m, Q}^{\prime} \leq C^{\prime}\|F\|_{m, Q}$ which completes the proof of the lemma.

After Lemma 9 we can give a similar characterization of forms $\left(\phi^{(r)}\right)_{r=0}^{m}$ which correspond to a Whitney function $F=\left(f_{\nu}\right)_{|\nu| \leq m}$ under the monomorphism (B.1).

Given a vector field $\eta=\sum_{1}^{n} \alpha_{i} \partial_{i} \in C^{m}\left(\Omega ; \mathbf{C}^{n}\right)(m \geq 1)$ and a tangent field $\xi \in \Gamma(\Omega ; \tau)$ we denote by $\nabla_{\xi}(\eta)$ the vector field $\sum_{1}^{n} \partial_{\xi}\left(\alpha_{i}\right) \partial_{i}$ (a covariant derivative of $\eta$ along $\xi$ ) and by $\nabla_{\xi}^{\prime}(\eta), \nabla_{\xi}^{\prime \prime}(\eta)$ the tangential and normal components of $\nabla_{\xi}(\eta)$.

COROLlary 4. A tuple $F=\left(f_{\nu}\right)_{|\nu| \leq m}$ of continuous $B$-valued functions $f_{\nu} \in$ $C(\Omega ; B)$ is a Whitney function if and only if:

(a)' Each $\phi^{(r)}$ is $(m-r)$-smooth $(0 \leq r \leq m)$ i.e.,

$$
\phi^{(r)} \in \Gamma^{m-r}\left(\Omega ; \mathcal{E}^{(r)} \otimes B\right) .
$$

(b) $)^{\prime}$ For any tangent field $\xi \in \Gamma^{\infty}(\Omega, \tau)$ and a tuple $\left(\xi_{1}, \ldots, \xi_{r}\right) \subset C^{\infty}\left(\Omega ; \mathbf{R}^{n}\right)$,

$$
\nabla_{\xi}\left[\phi^{(r)}\left(\xi_{1}, \ldots, \xi_{r}\right)\right]=\phi^{(r+1)}\left(\xi, \xi_{1}, \ldots, \xi_{r}\right)+\sum_{i=1}^{r} \phi^{(r)}\left(\xi_{1}, \ldots, \nabla_{\xi}\left(\xi_{i}\right), \ldots, \xi_{r}\right) .
$$

Notice that the difference

$$
\nabla_{\xi}\left[\phi^{(r)}\left(\xi_{1}, \ldots, \xi_{r}\right)\right]-\sum_{i} \phi^{(r)}\left(\xi_{1}, \ldots, \nabla\left(\xi_{i}\right), \ldots, \xi_{r}\right)
$$

is by definition a covariant derivative $\nabla_{\xi}\left[\phi^{(r)}\right]$ "lifted" to $r$-forms. Hence (B.10) can also be written as

$$
\nabla_{\xi}\left[\phi^{(r)}\right](\cdots)=\phi^{(r+1)}(\xi, \ldots) \quad \forall \xi \in \Gamma^{\infty}(\Omega, \tau) .
$$

Condition $\left(\mathrm{a}^{\prime}\right)$ is obviously equivalent to (a) of Lemma 9.

To check the equivalence of (b) and (b') we decompose vector fields $\xi_{1}, \ldots, \xi_{r}$ into linear combinations

$$
\xi_{i}=\sum_{1}^{n} \alpha_{i j} \partial_{j}, \quad \alpha_{i j} \in C^{\infty}(\Omega), \quad i=1,2, \ldots, r .
$$

Then apply $\phi^{(r)}$ to the tuple $\left(\xi_{1}, \ldots, \xi_{r}\right)$,

$$
\phi^{(r)}\left(\xi_{1}, \ldots, \xi_{r}\right)=\sum_{j_{1} \ldots j_{r}} f_{\nu\left(j_{1} \ldots j_{r}\right)}(x) \alpha_{1 j_{1}} \ldots \alpha_{r j_{r}} .
$$

Differentiating (B.12) along $\xi$ and rearranging the terms one easily shows that (b) implies $\left(b^{\prime}\right)$.

Conversely, let a collection of forms $\left(\phi^{(r)}\right)_{r=0}^{m}$ satisfy $\left(\mathrm{a}^{\prime}\right)$ and $\left(\mathrm{b}^{\prime}\right)$. Take a tuple of basic vector fields $\left(\partial_{1}, \ldots, \partial_{1}, \partial_{2}, \ldots, \partial_{2}, \ldots, \partial_{n}, \ldots, \partial_{n}\right)\left(\nu_{1}\right.$ times " $\partial_{1}$ "; $\nu_{2}$ times 
" $\partial_{2}$ ", etc.) and substitute for $\xi_{1}, \ldots, \xi_{r}$ in $\left(\mathrm{b}^{\prime}\right)$. Then by the definition of $\phi^{(r)}$, (B.2),

$$
\partial_{\xi}\left(f_{\nu}\right)=\phi^{(r+1)}\left(\xi, \partial_{1}, \ldots, \partial_{n}\right)=\sum_{i=1}^{n} \alpha_{i} f_{\nu+\delta_{i}}
$$

where $\xi=\sum_{1}^{n} \alpha_{i} \partial_{i}$. This proves Corollary 4 . After Corollary 4 we can complete the proof of Theorem 3 .

For this we shall rewrite conditions $\left(\mathrm{a}^{\prime}\right)$ and $\left(\mathrm{b}^{\prime}\right)$ of Corollary 4 for $(k, l)$-components of $\phi^{(r)}=\sum_{k+l=r} \phi_{k, l}$.

Recall that $\phi_{k, l}$ is obtained by restriction of $\phi^{(r)}$ onto the set of all tuples of $k$-tangent fields $\xi_{1}, \ldots, \xi_{k} \subset \Gamma(\Omega ; \tau)$ and $l$-normal fields $\xi_{k+1} \ldots \xi_{r} \in \Gamma(\Omega ; \mathcal{N})$. Decompose $\nabla_{\xi}\left(\xi_{i}\right)$ into the sum of a tangent field $\nabla_{\xi}^{\prime}\left(\xi_{i}\right)$ and a normal field $\nabla_{\xi}^{\prime \prime}\left(\xi_{i}\right)$ and substitute in (B.11). We get

$$
\begin{aligned}
\nabla_{\xi}\left[\phi_{k, l}\left(\xi_{1}, \ldots, \xi_{r}\right)\right]= & \phi_{k+1, l}\left(\xi, \xi_{1}, \ldots, \xi_{r}\right)+\sum_{i=1}^{r} \phi_{k, l}\left(\xi_{1}, \ldots, \nabla_{\xi}^{\prime}\left(\xi_{i}\right) \cdots \xi_{r}\right) \\
& +\sum_{i=1}^{r} \phi_{k, l}\left(\xi_{1}, \ldots, \nabla_{\xi}^{\prime \prime}\left(\xi_{1}\right) \cdots \xi_{r}\right)
\end{aligned}
$$

For $1 \leq i \leq k$ the $i$ th term in the second sum of (B.13) represents a certain $(k-1, l+1)$-form depending on $\phi_{k, l}$, while for $k+1 \leq i \leq r$ the $i$ th term in the first sum represents a $(k+1, l-1)$-form depending on $\phi_{k, l}$.

Formula (B.13) expresses a $(k+1, l)$-component of the tuple $\left(\phi_{k, l}\right)_{k+l \leq m}$ corresponding to a Whitney function $F=\left(f_{\nu}\right)_{|\nu| \leq m}$ in terms of $\phi_{k, l}$. Hence the whole tuple $\left(\phi_{k, l}\right)_{k+l \leq m}$ is uniquely determined by $(m+1)$ its normal components $\left\{\phi_{0, l}\right\}_{l=0}^{m}$ which by $\left(\mathrm{a}^{\prime}\right)$ can be chosen independently in the spaces $\phi_{0, l} \in \Gamma^{m-l}\left(\Omega ; \mathcal{E}_{0, l} \otimes B\right)$. This proves the first statement of Theorem 3 and also the equivalence of norms on the space of Whitney functions, $\|F\|_{m}^{\prime} \sim\|F\|_{m}$.

Now we want to show that the isomorphism (B.1) is topological, i.e., the $m$ norm (5.4.5) on the space of Whitney functions $C^{m}(\Omega ; B)$ is equivalent to the sum of $(m-l)$-norms (5.3.4) on the spaces of sections $\Gamma^{m-l}\left(\Omega ; \mathcal{E}_{l} \otimes B\right)$.

We consider a Whitney function $F=\left(f_{\nu}\right)_{|\nu| \leq m}$ and a tuple of forms $\phi^{(r)} \in$ $\Gamma^{m-r}\left(\Omega ; \mathcal{E}^{(r)} \otimes B\right)(r=0,1, \ldots, m)$, which corresponds to $F$ according to Theorem 3.

In one direction the inequality between norms is easy. Indeed, a coefficient $f_{\nu}$ is the "value" of a multilinear form $\phi^{(r)}$ on the tuple of basic vector fields $\partial_{1}\left(\nu_{1}\right.$ times), $\partial_{2}\left(\nu_{2}\right.$-times) etc. $(|\nu|=r),(\mathrm{B} .2)$. So we get

$$
\left\|f_{\nu}\right\|_{L^{\infty}}=\left\|\phi^{(r)}\left(x ; \partial_{1}, \partial_{1}, \ldots, \partial_{n}\right)\right\|_{L^{\infty}} \leq\left\|\phi^{(r)}\right\|_{L^{\infty}} .
$$

Hence

$$
\|F\|_{m}=\sum_{|\nu| \leq m}\left\|f_{\nu}\right\|_{L^{\infty}} \leq \sum_{r=0}^{m}\left(\begin{array}{c}
n+r+1 \\
n+1
\end{array}\right)\left\|\phi^{(r)}\right\|_{L^{\infty}} \leq c \sum_{r=0}^{m}\left\|\phi^{(r)}\right\|_{\Gamma^{(m-r)}} .
$$

To prove the inverse inequality we need explicit formulas for the action of infinitesimal operators $L_{h}(h \in \mathfrak{H})$ on sections $\phi^{(r)} \in \Gamma^{m-r}\left(\Omega ; \mathcal{E}^{(r)} \otimes B\right)$. Note that 
the bundle $\mathcal{E}^{(r)}=S_{r}\left(\mathbf{R}^{n}\right)$ is trivial. Therefore infinitesimal operators $L_{h}(h \in \mathfrak{H})$ have the form (5.3.8), i.e.,

$$
L_{h} \phi^{(r)}=\partial_{\xi(h)} \phi^{(r)}+B(h, \cdot) \phi^{(r)} .
$$

Regarding $\phi^{(r)}$ as a multilinear form we write the first term of (B.14) as

$$
\left(\partial_{\xi(h)} \phi^{(r)}\right)\left(\eta_{1}, \ldots, \eta_{r}\right)=\partial_{\xi(h)}\left[\phi^{(r)}\left(\eta_{1}, \ldots, \eta_{r}\right)\right]-\sum_{1}^{r} \phi^{(r)}\left(\cdots \nabla_{\xi}\left(\eta_{i}\right) \cdots\right)
$$

$\forall \eta_{1}, \ldots, \eta_{r} \in C^{\infty}\left(\Omega ; \mathbf{R}^{n}\right)$, which by Corollary $4\left(\mathrm{~b}^{\prime}\right)$ is equal to

$$
\phi^{(r+1)}\left(\xi(h), \eta_{1}, \ldots, \eta_{r}\right) \text {. }
$$

Obviously,

$$
\left\|\partial_{\xi(h)} \phi^{(r)}\right\|_{L^{\infty}} \leq c\left\|\phi^{(r+1)}\right\|_{L^{\infty}}
$$

with some constant $c$ depending only on $h$.

The second term of (B.14) is estimated in a similar way,

$$
\left\|B(h, \cdot) \phi^{(r)}\right\|_{L^{\infty}} \leq\|B(h, \cdot)\|_{L^{\infty}}\left\|\phi^{(r)}\right\|_{L^{\infty}} .
$$

Hence,

$$
\left\|L_{h} \phi^{(r)}\right\|_{L^{\infty}} \leq c^{\prime}\left(\left\|\phi^{(r+1)}\right\|_{L^{\infty}}+\left\|\phi^{(r)}\right\|_{L^{\infty}}\right) .
$$

Iterating the latter inequality $k$ times $(k \leq m-r)$ we get

$$
\left\|L_{h_{1}} \cdots L_{h_{k}} \phi^{(r)}\right\|_{L^{\infty}} \leq c \sum_{j=0}^{k}\left\|\phi^{(r+j)}\right\|_{L^{\infty}} \quad \forall h_{1}, \ldots, h_{k} \in \mathfrak{H}
$$

with some constant $c$ depending on $\left(h_{1}, \ldots, h_{k}\right)$.

The $L^{\infty}$-norm of each $\phi^{(r)}$ is estimated by the $L^{\infty}$-norms of its coefficients (B.2)

$$
\left\|\phi^{(r)}\right\|_{L^{\infty}} \leq \sum_{|\nu|=r}\left\|f_{\nu}\right\|_{L^{\infty}}
$$

Thus we get

$$
\begin{aligned}
\sum_{r=0}^{m}\left\|\phi^{(r)}\right\|_{\Gamma^{(m-r)}} & =\sum_{r} \sum_{|\nu| \leq m-r}\left\|L_{n}^{\nu} \phi^{(r)}\right\|_{L^{\infty}} \\
& \leq C \sum_{|\mu| \leq m}\left\|f_{\mu}\right\|=C\|F\|_{m} . \quad \text { Q.E.D. }
\end{aligned}
$$

\section{REFERENCES}

1. R. C. Busby and M. A. Smith, Representations of tursted group algebras, Trans. Amer. Math. Soc. 149 (1970), 503-537.

2. Y. Domar, Harmonic analysis based on certain commutative Banach algebras, Acta Math. 96 (1956), $1-66$.

3. $\ldots$, On the spectral synthesis problem for $(n-1)$ dimensional subsets of $\mathbf{R}^{n}, n \geq 2$, Ark. Mat. 9 (1971), 23-37.

4. J. M. G. Fell, Nonunitary dual spaces of groups, Acta Math. 144 (1965), 267-310.

5. _ Induced representations and Banach ${ }^{*}$-algebraic bundles, Lecture Notes in Math., vol. 582, Springer-Verlag, Berlin and New York, 1977.

6. I. Gelfand, D. Raikov and G. Shilov, Commutative normed rings, Chelsea, New York, 1964. 
7. R. Godement, $A$ theory of spherical functions. I, Trans. Amer. Math. Soc. 73 (1952), 496-556.

8. S. Grosser and M. Moskowitz, Compactness conditions in topological groups, J. Reine Angew. Math. 246 (1971), 1-40.

9. D. Gurarie, Harmonic analysis based on crossed product algebras and motion groups, Proc. Conf Harmonic Analysis, Iraklion, Lecture Notes in Math., vol. 781, Springer-Verlag, Berlin and New York, 1980.

10. ___ Burnside type theorems for Banach representations of motion groups and algebras. CMS Conference Proceedings, vol. 1, Amer. Math. Soc., Providence, R.I., 1982, pp. 213-244.

11. E. Hewitt and K. Ross, Abstract harmonic analysis, vol. 2, Springer-Verlag, Berlin and New York, 1970.

12. W. Kirsch and D. Müller, Zum Symthese problem für Bahnen Lie Scher Gruppen in $\mathbf{R}^{n}$, preprint, 1979.

13. H. Leptin, Verallgemeinerte $L^{1}$-Algebren und projective Darstellungen lokal kompacter Gruppen. I, Invent. Math. 3 (1967), 257-281; II, Invent. Math. 4 (1967), 68-86.

14. $\ldots$ On one sided harmonic analysis in noncommutative locally compact groups, J. Reine Angew. Math 306 (1979), 122-153.

15. J. Liukkonen and R. Mosak, Harmonic analysis and centers of Beurling algebras, Comment Math. Helv. 52 (1977), 297-315.

16. B. Malgrange, Ideals of differentiable functions, Oxford Univ. Press, London and New York, 1966.

17. C. C. Moore, On the Frobenius reciprocity theorem for locally compact groups, Pacific J. Math. 12 (1962), 359-365.

18. R. D. Mosak, Ditkin's condition and primary ideals in central Beurling algebras, Monatsh. Math. 85 (1978), 115-124.

19. L. C. Pontrjagin, Continuous groups, English transl., Gordon \& Breach, New York, 1966.

20. H. J. Reiter, Contributions to harmonic analysis, Acta Math. 96 (1956), 253-263.

21. M. S. Riffel, Induced Banach representations of Banach algebras and locally compact groups, J. Funct. Anal. 1 (1967), 443-491.

22. W. Rudin, Fourier analysis on groups, Interscience, New York, 1962.

23. Séminaire "Sophus Lie" de L'Ecole Normale Supérieure 1954/55, Théorie des algèbres de Lie. Topologie des groupes de Lie, Secrétariat Math., Paris, 1955.

24. N. T. Varopoulos, Spectral symthesis on spheres, Proc. Cambridge Philos. Soc. 62 (1966), 379 387.

25. N. R. Wallach, Harmonic analysis on homogeneous spaces, Dekker, New York, 1973.

26. H. Whitney, On ideals of differentiable functions, Amer. J. Math. 70 (1948), 635-658.

Department of Mathematics, Case Western Reserve University, CleveLAND, OHIO 44106 